\title{
The dynamics of Brazilian protozoology over the past century
}

\author{
M Carolina Elias ${ }^{1,2}{ }^{+}$, Lucile M Floeter-Winter ${ }^{3}$, Jesus P Mena-Chalco ${ }^{4}$

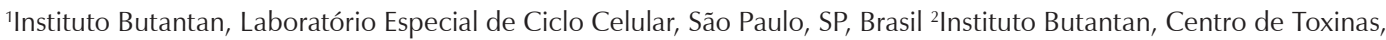 \\ Resposta-Imune e Sinalização Celular, São Paulo, SP, Brasil ' ${ }^{3}$ Universidade de São Paulo, Instituto de Biociências, Departamento de Fisiologia, \\ São Paulo, SP, Brasil ${ }^{4}$ Universidade Federal do ABC, Centro de Matemática, Computação e Cognição, Santo André, SP, Brasil
}

Brazilian scientists have been contributing to the protozoology field for more than 100 years with important discoveries of new species such as Trypanosoma cruzi and Leishmania spp. In this work, we used a Brazilian thesis database (Coordination for the Improvement of Higher Education Personnel) covering the period from 1987-2011 to identify researchers who contributed substantially to protozoology. We selected 248 advisors by filtering to obtain researchers who supervised at least 10 theses. Based on a computational analysis of the thesis databases, we found students who were supervised by these scientists. A computational procedure was developed to determine the advisors' scientific ancestors using the Lattes Platform. These analyses provided a list of 1,997 researchers who were inspected through Lattes $C V$ examination and allowed the identification of the pioneers of Brazilian protozoology. Moreover, we investigated the areas in which researchers who earned PhDs in protozoology are now working. We found that $68.4 \%$ of them are still in protozoology, while $16.7 \%$ have migrated to other fields. We observed that support for protozoology by national or international agencies is clearly correlated with the increase of scientists in the field. Finally, we described the academic genealogy of Brazilian protozoology by formalising the "forest" of Brazilian scientists involved in the study of protozoa and their vectors over the past century.

Key words: protozoology - pioneers - academic genealogy - scientific mapping method

The last decade of the XIX century is considered to be the period in which experimental protozoology began (Calkins 1911). At that time, protozoa were suspected of being the causative agents of only two human diseases: dysentery and malaria (Calkins 1911). Currently, protozoan parasites are recognised as the causative agents of some of most important human illnesses. For instance, amebiasis is the second leading cause of death due to parasitic diseases worldwide and causes approximately 40-100,000 deaths per year (Moraes et al. 2015). Approximately 6.5 million people are estimated to be infected with Trypanosoma cruzi, 1,300,000 new cases of leishmaniasis occur every year, and 214 million new cases of malaria have occurred in 2015 alone. Toxoplasmosis and giardiasis are also diseases caused by protozoa, both of which represent significant public health threats (who.int). These are examples of protozoa that are of medical interest and do not include protozoa of veterinary interest or free-living protozoa, which may be important environmental markers.

Therefore, protozoology has become a unique field of study, and an impressive amount of work has gone into detailing the biological aspects of this subject. In-

doi: 10.1590/0074-02760150386

Financial support: FAPESP (2013/07467-1 to MCE), CNPq (461757/2014-1 to JPM-C)

MCE and LMF-W are research fellows from CNPq (306376/2012-1

and 307587/2014-2 respectively).

+ Corresponding author: carolina.eliassabbaga@butantan.gov.br

Received 8 October 2015

Accepted 8 December 2015 deed, the study of protozoa has rapidly evolved from the molecular characterisation of eukaryotes through hostparasite interactions and ecoepidemiological aspects to therapeutic interventions. Several important mechanisms were first described in protozoa, such as nonconventional RNA polymerase II promoter sites (Clayton 2002), trans-splicing (Mayer \& Floeter-Winter 2012) and RNA editing (Simpson et al. 2006). Some of these findings have been highly influential in other medical and biological fields, such as the discovery of telomeres protecting chromosomes in Tetrahymena (Blackburn \& Gall 1978), the understanding of glycosylphosphatidylinositol protein anchor structures (Ferguson et al. 1985, Ferguson 1999), and the delineation of the respective roles of the T-helper (Th)1 and Th2 lymphocyte subsets against infectious agents in studies using Leishmania major-infected mice (Heinzel et al. 1989).

In Brazil, where some protozoan infections are endemic, these aetiological agents and their vectors have attracted considerable interest from researchers and students. A few "founding fathers" (pioneers) of this field of science have nurtured later generations of protozoologists, resulting in the development of a solid network of 100 years of scientists.

The evolution of science is the pillar that provides a solid foundation for the development of society by creating the means to face the challenges ahead (Cordova et al. 2015). The study of the origin of a scientific area and the identification of the motives behind its development in new disciplines provide important contributions to the understanding of future needs. This academic genealogy allows the development of qualifying studies based on the training of new researchers.

Academic genealogy was defined by Sugimoto (2014) as a quantitative study of the intellectual heritage 


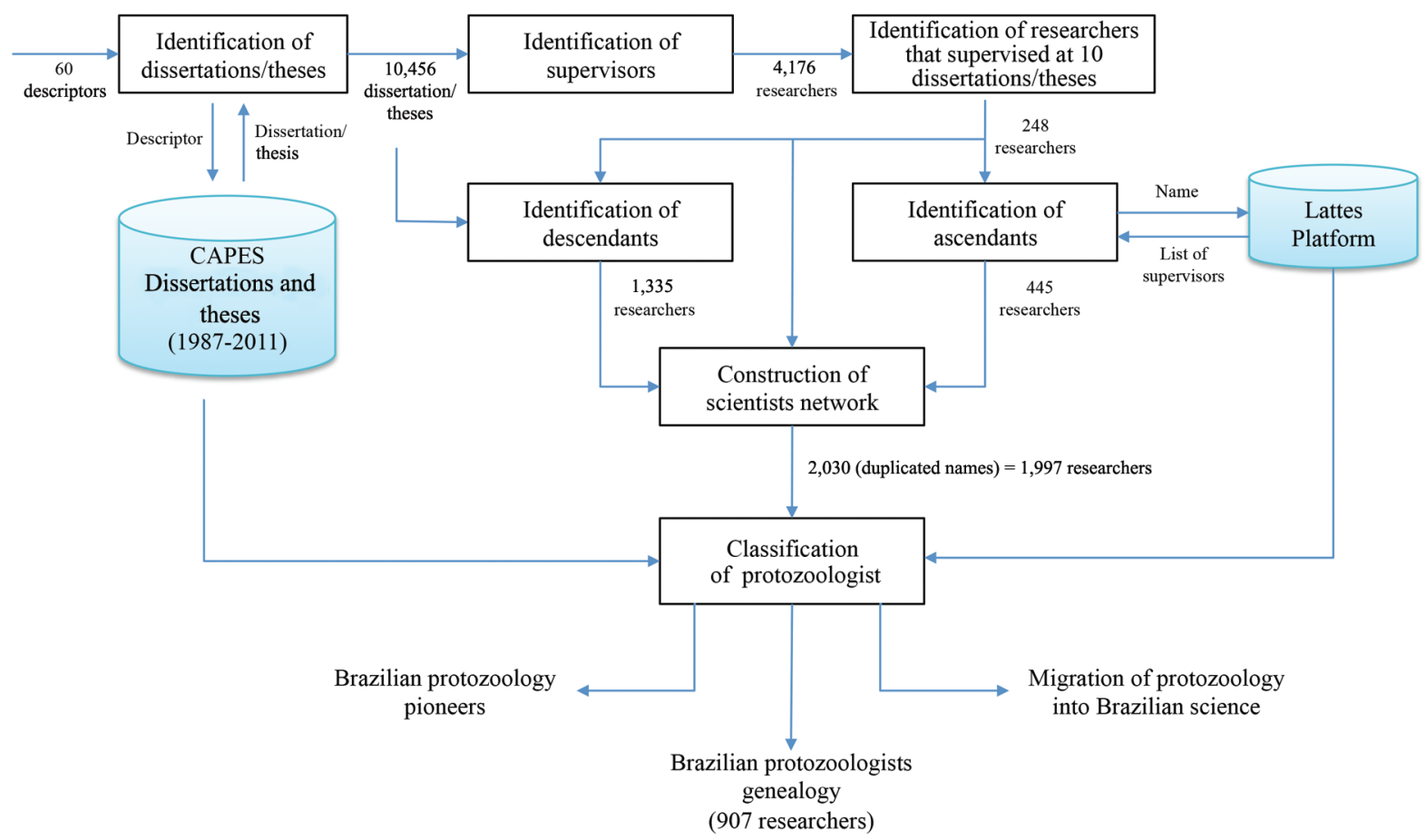

Fig. 1: diagram of the tracking of Brazilian protozoologists. Each block represents a process and each arrow represents the information flow between processes.

perpetuated through academic orientation relationships among professors (i.e., mentors or supervisors) and their students. Various questions can be answered by building an academic genealogy. Sugimoto (2014) proposed five types of academic genealogies: honorific, egotistical, historical, paradigmatic, and analytical. These categories are not mutually exclusive, and most academic genealogies can be classified into at least two of these types.

In this work, we followed the development of protozoology in Brazil using a historical and paradigmatic approach. Systematic data collection was performed through the analysis of formal thesis orientation and was organised to create a chain of mentorships, resulting in the construction of our academic genealogy.

\section{MATERIALS AND METHODS}

In this work, the identification of researchers associated with protozoology who influenced (supervised) other researchers over a 100 year timespan was performed through seven processes (Fig. 1). The methodology was based on the analysis of two sources of Brazilian academic information that allowed the tracing of scholarly interactions among researchers.

Process 1 - Identification of dissertations and theses related to protozoology - A local repository extracted from the Coordination for the Improvement of Higher Education Personnel (CAPES) thesis database (Mena-Chalco \& Rocha 2014) was used in this process. We identified 10,456 dissertations or theses with at least one descriptor related to protozoology (Supplementary Table I). This quantity represented $1.7 \%$ of the 607,389 dissertations or theses registered in the local repository.

Process 2 - Identification of supervisors of Masters and PhD students in protozoology - In this process, we extracted all supervisors' names from the dissertations/ theses identified in process 1 . The process handled incomplete or similar names using approximate string matching. Two names were considered the same/similar if the Levenshtein distance (Levenshtein 1966) between them was equal to 2 . This process allowed us to identify 4,176 researchers related to the protozoology field.

Process 3 - Identification of representative protozoologists in terms of the quantity of supervisions registered in the CAPES thesis database - In this process, researchers who supervised at least 10 projects were selected to generate a list of 248 supervisors $(6 \%$ of 4,176 researchers). This threshold was considered suitable for manual inspection. Supplementary Table II presents the complete list of names obtained through this process.

Process 4 - Identification of ancestors of the representative protozoologists - This process was performed recursively for each supervisor identified in the Lattes Platform. First, the name of the supervisor was identified and associated with its Lattes CV. Then, the same approach was undertaken for the supervisors of the supervisors until the inability to identify a new supervisor was reached. We identified 445 researchers as the ancestors of the 248 representative protozoologists. 
Process 5 - Identification of the descendants of the representative protozoologists - This process was accomplished by selecting the $\mathrm{PhD}$ students (process 1) who were supervised by the researchers obtained in process 3 . We identified 1,335 direct descendants from the group of 248 representative protozoologists.

Process 6 - Construction of a scientist network - In this process, the researchers identified in processes 3,4 and 5 were used to generate a supervisor network (i.e., a directed graph where each node represented a researcher, and the edge represented the relationship between 2 researchers). Duplicated names were processed manually. A list of 1,997 complete names was generated as the result of this process (Supplementary Table III).

Process 7 - Classification of protozoologists - This process was performed by manual inspection of each researcher identified in the previous process. Information from the protozoology field was associated with each of the 1,997 researchers using the Lattes Platform and academic repositories.

\section{RESULTS}

Identification of scientists actively working in protozoology - This work aims to understand the past and present of Brazilian protozoology and the migration of scientists from protozoology to other fields and from other fields to protozoology. Academic dissertations and theses are an important source of information concerning the growth and evolution of science (Andersen \& Hammarfelt 2011). Therefore, we accessed a data collection containing most of the Brazilian protozoologists from the CAPES database combined with the Lattes examination. By searching for the supervisors of actively working protozoologists, the supervisors of these supervisors, and so on, we identified the pioneers of this field in Brazil. Similarly, by searching for students trained by these actively working protozoologists, we identified other people currently working in the field. To establish the names of actively working protozoologists to nucleate our search, we prepared a set of 60 words (descriptors) (Supplementary Table I) for use as keywords to screen theses (Masters or PhD) present in the local CAPES thesis database, which contained all theses completed in Brazil from 1987-2011 (Mena-Chalco \& Rocha 2014). A total of 10,456 Masters or PhD theses contained at least one such descriptor. This number corresponded to $1.7 \%$ of the total theses presented in the same period throughout all fields. A total of 4,176 researchers supervised these 10,456 dissertations/theses. As expected, the number of scientists diminished when we increased the number of works supervised per researcher (Fig. 2). For operational reasons, it might not be possible to analyse in detail all the 4,176 researchers (advisors) and the relevant factors considered in the adopted methodology. Therefore, only researchers with at least 10 dissertations/theses supervised in the field of protozoology were considered in our study (248 scientists) (Supplementary Table II). With a threshold of five or two dissertations, for instance, the number of researchers increases by $276 \%$ (689 researchers) or $745 \%$ (1,864 researchers), respec-

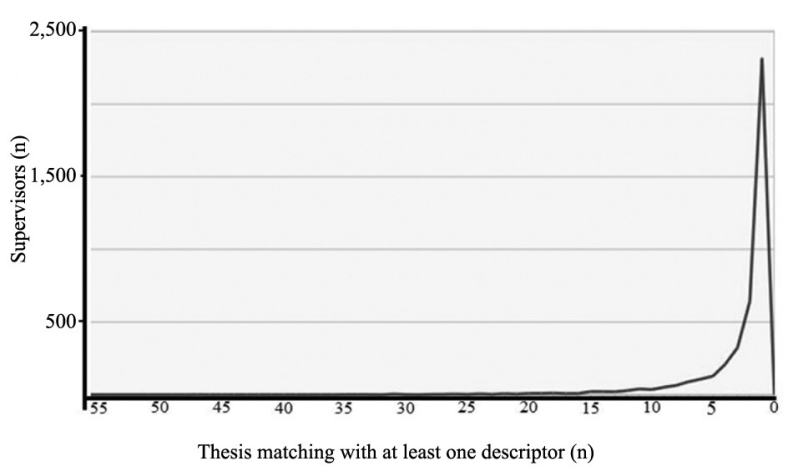

Fig. 2: relationship between the number of supervisors and number of theses generated from 1987-2011 containing at least one descriptor. Supplementary Table I containing 60 descriptors was used to screen the Coordination for the Improvement of Higher Education Personnel database. The graph indicates how many scientists supervised a different number of theses containing at least one descriptor as a keyword.

tively. With the threshold adopted, important pioneers in the field of protozoology are included in the analyses, but we recognise that this empirical value might penalise early career researchers. However, we believe that this arbitrarily selected threshold of 10 dissertations/theses was appropriate to define a selected group of representative researchers. A total of $36 \%$ of these researchers are or were members of the Brazilian Society of Protozoology in the period between 2000-2015.

Identification of Brazilian protozoologists - We used the complete list of names in Supplementary Table II as a starting point to search for the people supervised by these people and vice versa (i.e., scientists who supervised these people). Using automated analysis of the local CAPES thesis database, we found students whom these researchers supervised (descendants). We did not search for students of these students because some of them were still settling, which could compromise our analysis. Based on the automated analysis of their Lattes CVs, we could also follow the advisors' ancestors by searching for their supervisors and the supervisors of these supervisors available on the Lattes CV database (Fig. 1). As shown below, the analyses also allowed the identification of Brazilian supervisors who worked outside of protozoology and supervisors that were from other countries; thus, we could identify the seeds of Brazilian protozoology. These analyses provided a list of 1,997 names that were manually inspected through the Lattes CV examination, following the pathway shown in Fig. 3A. We used the following criteria to establish that a researcher is/was working in the field of protozoology. Researchers studying the biology of protozoa or protozoahost interaction were considered protozoologists. Additionally, scientists investigating protozoa vectors were considered protozoologists. In contrast, researchers working with protozoa but investigating the clinical aspects of diseases (e.g., mostly ophthalmologists, dermatologists, and cardiologists who study diseases caused by protozoa) were not considered protozoologists. From the total of 


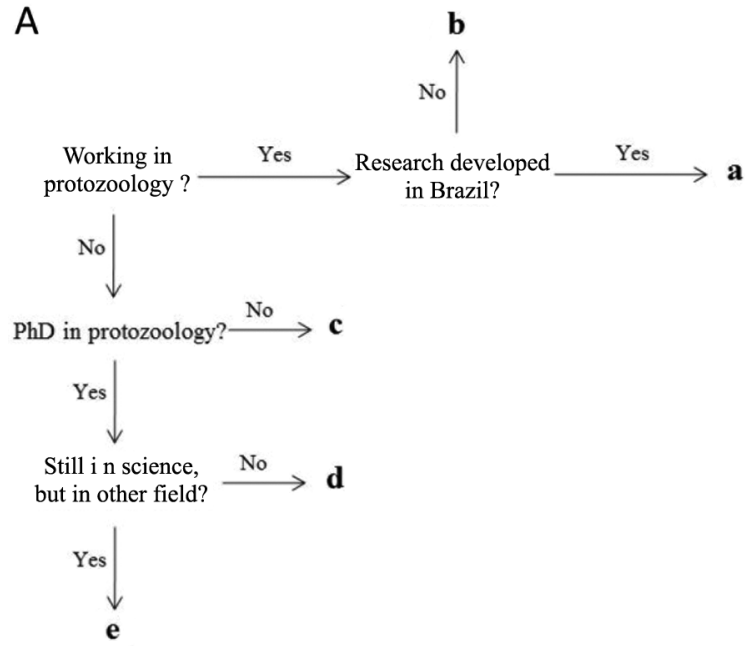

Fig. 3: classification of scientists according to Lattes CV. A: pathway followed during inspection with Lattes CV; B: frequency of each category described in A; a: scientists working or who worked in the past in protozoology in Brazil; b: scientists working in protozoology outside of Brazil that were advisors of Brazilians; c: scientists that did not work in protozoology during their PhD or establish a research interest in this field but were advisors of scientists that migrated to protozoology; $\mathrm{d}$ : persons who developed their $\mathrm{PhD}$ in protozoology but are now involved in activities other than science; e: scientists who developed their PhD in protozoology but established research in another field.

1,997 names found, some were not included in the Lattes database. This discrepancy might have occurred for four different reasons: (i) misspelling of names, (ii) people who are no longer in science, (iii) foreign supervisors, and (iv) people who worked in protozoology before the Lattes CV was created. To include this last group in our analysis, we searched for these people in the CAPES database or in public repositories to identify their ancestors and the field in which they worked. To include supervisors from other countries, we searched for these people in PubMed to verify the field in which they work/worked. Finally, $10.86 \%$ of the 1,997 names could not be found in either the Lattes Platform, CAPES database, or public repositories and were removed from the forward analyses. These analyses allowed the classification of researchers into five different categories: (a) scientists who are working or previously worked in protozoology in Brazil, (b) scientists working in protozoology outside of Brazil who were advisors of Brazilians, (c) scientists who did not work in protozoology during their $\mathrm{PhD}$ and did not establish a research interest in this field but were advisors of scientists who migrated to protozoology, (d) people who obtained their PhD in protozoology but were now involved in activities other than science, and (e) scientists who developed their $\mathrm{PhD}$ in protozoology but established a research interest in another field (Supplementary Table III). The frequency of each group is presented in Fig. 3B. Based on the data, 907 names were identified as Brazilian protozoologists (classified as group a).

Identification of Brazilian protozoology pioneers - To identify researchers who were Brazilian protozoology pioneers, we first assumed that people who were awarded a $\mathrm{PhD}$ in protozoology in Brazil were not pioneers but that the pioneers would be their advisors.
Therefore, we concluded that the pioneers were among the scientists who were awarded $\mathrm{PhD}$ degrees in other fields and then migrated to protozoology or people who were awarded PhDs in protozoology outside Brazil and then came to our country to establish a group here. Among the researchers classified as group a (Brazilian protozoologists), we manually searched the Lattes $\mathrm{CV}$ and the CAPES database for ones who were (766) and were not (141) awarded a PhD in protozoology (Fig. 4, Supplementary Table IV). Additionally, we searched the Lattes CV for researchers who were awarded a $\mathrm{PhD}$ in protozoology outside Brazil (15) (Supplementary Table $\mathrm{V}$, in bold). Then, we determined in what year these researchers who did not receive a $\mathrm{PhD}$ in protozoology (141

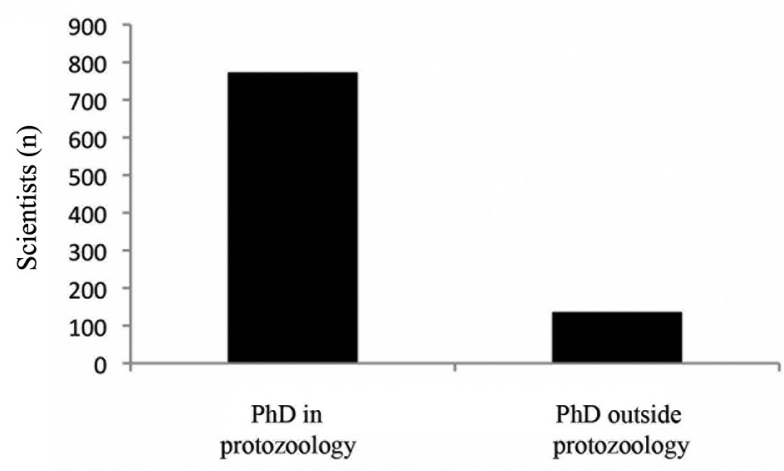

Fig. 4: analysis of fields where scientists classified as developed their PhD. Scientists classified in category "PhD in protozoology" were divided into those who developed and those who did not develop PhDs in protozoology. 
A

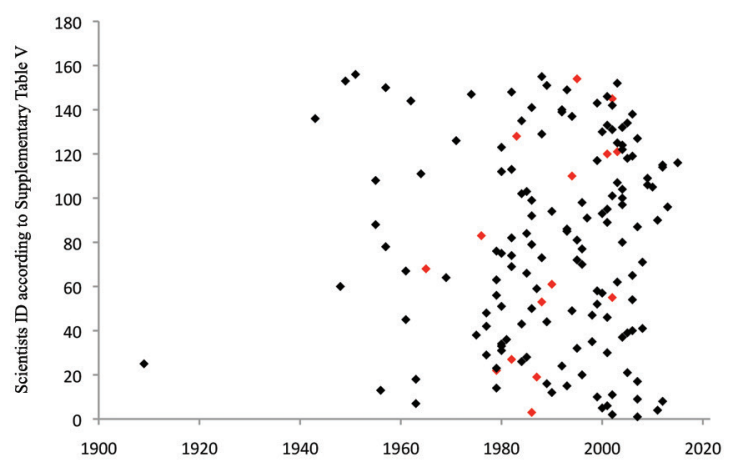

B

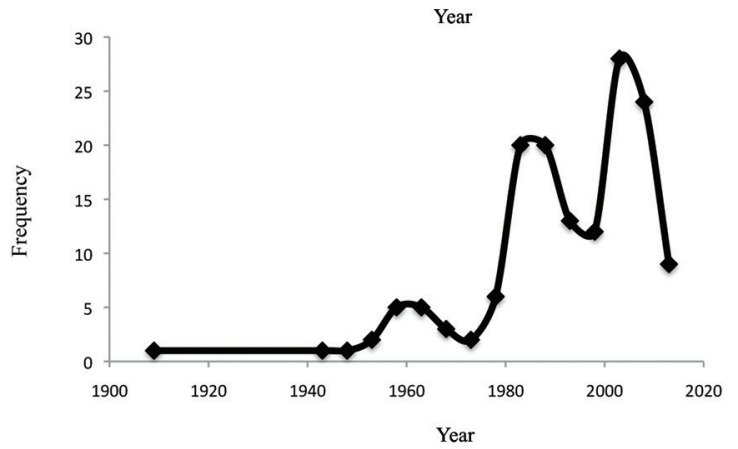

Fig. 5: incoming scientists into protozoology. A: the graph shows the year in which each researcher who did not develop a PhD in protozoology published their first paper in this field. Red dots indicate the year that scientists who developed a $\mathrm{PhD}$ in protozoology outside of Brazil published their first paper in Brazil after their $\mathrm{PhD}$; B: frequency of scientists immigrating into Brazilian protozoology according to the year of arrival.

names) or who received a PhD outside Brazil (15 names; a total of 156 names) established a protozoology group in Brazil. For scientists who arrived from other fields, we searched the Lattes CV for the year of their first paper published in protozoology (Supplementary Table V). For scientists who received their PhD outside of Brazil, we searched the Lattes CV for the year in which they published their first paper after their $\mathrm{PhD}$ from a position in Brazil (Supplementary Table V, in bold). Carlos Chagas's paper describing T. cruzi was not found in PubMed but was considered due to its relevancy. Using this date as the year the scientist entered Brazilian protozoology, we plotted a graph of this incoming year for each of the 156 scientists who arrived in protozoology (Fig. 5A). Additionally, we plotted a graph showing the frequency of this influx according to the year of migration (Fig. 5B). From both analyses, it was clear that there were three waves of immigration into Brazilian protozoology: (i) up to and including 1974, (ii) between 1978-1993, and (iii) between 1998-2013. However, the drop observed after 2013 might be artificial because the collection of data is recent. Thus, we concluded that the pioneers (i.e., founders or precursors) of Brazilian protozoology were the 20 scientists who migrated into the field up to 1974: Carlos
Chagas, Samuel Pessoa, Hertha Meyer, Zigman Brener, Wladimir Lobato Paraense, Leonidas Deane, Maria von Paumgartten Deane, Amilcar Viana Martins, José Rodrigues da Silva, Washington Luiz Tafuri, Erney Felicio Plessmann de Camargo, Jayme Neves, Aluízio Prata, Thales de Brito, Astolpho Ferraz de Siqueira, Jeffrey Jon Shaw, Mario Endsfeldez Camargo, Isaac Roitman, Raymundo Martins de Castro, and Walter Colli.

Migration of protozoology into Brazilian science We also investigated the immigration and emigration of scientists to and from Brazilian protozoology. A total of $17.1 \%$ of the studied researchers immigrated into protozoology, based on the number of people who entered protozoology from other fields and arrived from protozoology outside of Brazil (156 names/907 total). Researchers who stayed in protozoology after their $\mathrm{PhD}$ corresponded to $68.4 \%$ of individuals, based on the 770 protozoologists who received a $\mathrm{PhD}$ in protozoology and were still acting as protozoologists, those who received a $\mathrm{PhD}$ in protozoology and were involved in other activities but still involved in science [group d: 167 (Supplementary Table III)], and those who received a $\mathrm{PhD}$ in protozoology and established a group in another field [group e: 188 (Supplementary Table III)]. Finally, we determined the percentage of people who left Brazilian protozoology to contribute to other fields of Brazilian science. To obtain this number, we divided the number of people classified as group e in Supplementary Table III (188) by the total number who received a $\mathrm{PhD}$ in protozoology. This analysis demonstrated that protozoology provided $16.7 \%$ of its PhDs to other fields of science.

Academic genealogy of Brazilian protozoologists Finally, we constructed an academic genealogy (Fig. 6) that included all names classified as protozoologists to reflect the scenario of protozoology in Brazil. The resulting structure is a forest containing one tree for each scientist who entered Brazilian protozoology. A detailed view of this academic genealogy is available from professor.ufabc.edu.br/ jesus.mena/brazilian-protozoology-scenario/. It is clear that protozoology expanded in the mid-1970s. It is also clear that protozoology today consists of the descendants of the pioneers as well as other scientists who migrated to protozoology and supervised their students in this field.

\section{DISCUSSION}

The analysis of the dynamics of Brazilian protozoology over the past century based on database searches from 1987-2011 allowed us to track and identify scientists who made important contributions by acting as the pioneers of this field. Moreover, we could determine the percentage of scientists immigrating to protozoology as well as the percentage of scientists who were supervised in protozoology but established a research group in another field. The field of Brazilian protozoology comprises at least 907 researchers. Certainly, there are other protozoologists who were not covered by our criteria and therefore were not included in our analysis. The interesting result is that a large number of people working in this field are the result of the efforts of 20 pioneers who introduced protozoa as 


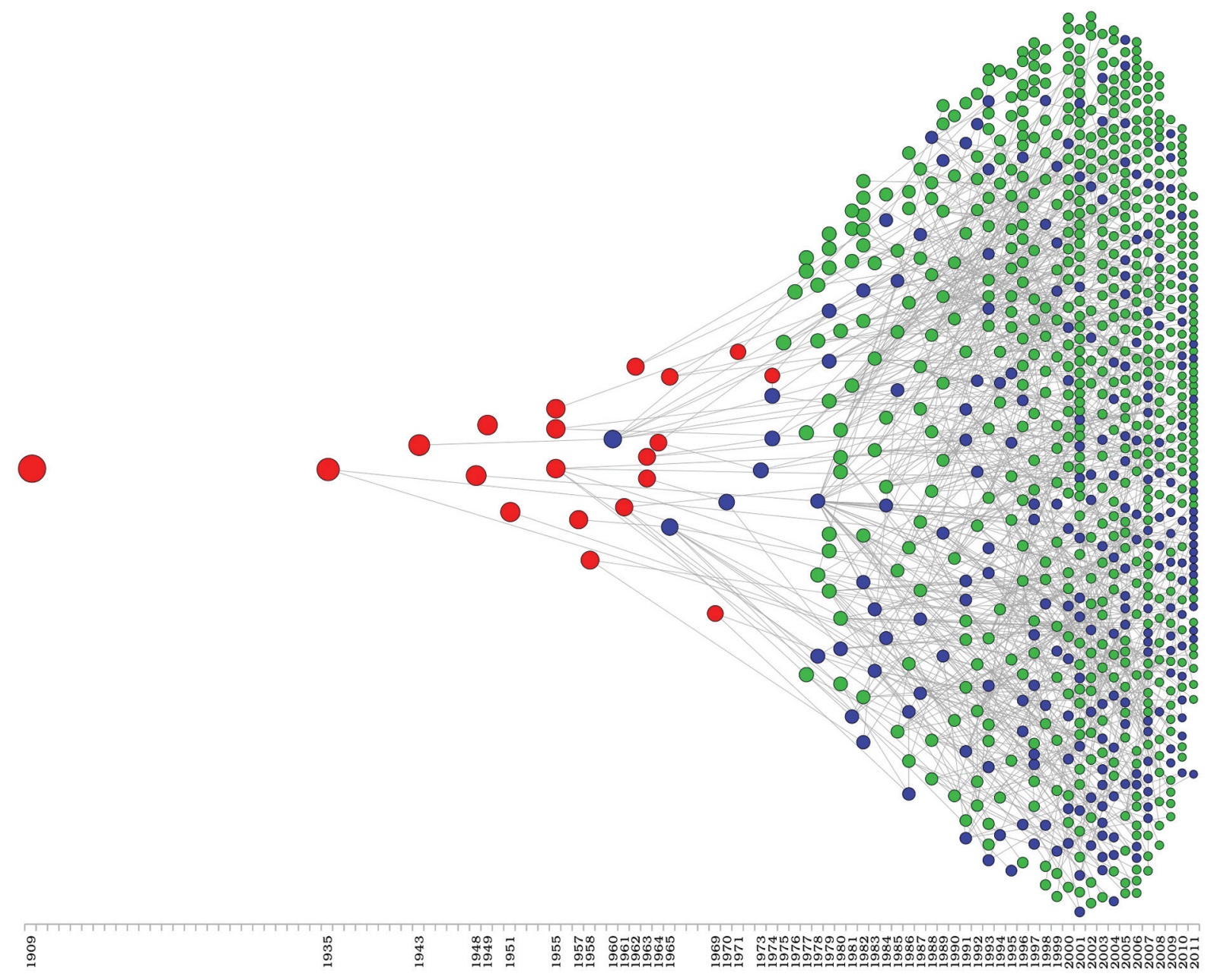

Fig. 6: academic genealogy of Brazilian protozoology protozoologists (group a in Fig. 3) were included in this graph. Each circle represents one researcher and lines represent student-supervisor relationships. Persons identified as pioneers are shown in red, persons directly influenced by pioneers (students or students of their students) are shown in blue, and persons who arrived in protozoology after the pioneers and their descendants are shown in green. The $\mathrm{x}$-axis shows the year of the conclusion of the $\mathrm{PhD}$ in protozoology or, for those who did not receive a $\mathrm{PhD}$ in protozoology, the year that they migrated to protozoology.

biological models or drug targets in the period between 1909-1974. In a seminal paper published in Memórias do Instituto Oswaldo Cruz, Carlos Chagas described T. cruzi and thereby introduced the study of protozoology in Brazil (Chagas 1909). Surprisingly, no descendants were identified for Carlos Chagas, probably because his descendants were clinical doctors and therefore were not classified as protozoologists according to the criteria used in this study. However, Carlos Chagas's work at the beginning of the previous century was so complete in showing the causal agent of a disease and its relationship to an insect vector that it still inspires researchers today.

In the 1940s, Samuel Pessoa inaugurated his huge contribution to the field by studying the behaviour of Leishmania in tissues (Pessoa \& Barreto 1945) and the geographic distribution of phlebotomines (Barreto \& Pessoa 1946). Hertha Meyer published on T. cruzi cul- tivation (Meyer \& de Oliveira 1948); this field of study evolved to include her contribution regarding the structural analysis of this organism. In the 1950s, the study of protozoa in Brazil expanded with Amilcar Viana Martins, Leonidas Deane, and Maria Deane, who investigated the epidemiology of leishmaniasis (Deane \& Deane 1954, Martins et al. 1956), Zigman Brener and Washington Luiz Tafuri, who contributed to our understanding of Chagas disease (Brener 1952, de Queiroz \& Tafuri 1957), José Rodrigues da Silva, who investigated hepatic problems related to Leishmania and amoeba infections (da Silva \& de Paola 1957, da Silva \& Torres 1957), and Wladimir Lobato Paraense, who was interested in Plasmodium (Paraense 1952). Then, over the next two decades, a new group of scientists migrated into protozoology. Their studies included the detection and treatment of Trypanosoma, Leishmania, Toxoplasma, 
and Plasmodium by Raymundo Martins de Castro (Sampaio et al. 1971), Aluízio Prata (Prata 1963), Mario Camargo (Camargo 1964b), and Thales de Brito (de Brito et al. 1962), the biology of trypanosomatids by Walter Colli (Alves \& Colli 1974), Erney Camargo (Camargo 1964a), and Isaac Roitman (Roitman 1969), and epidemiological approaches to studying diseases and hosts by Jeffrey Shaw (Shaw \& Lainson 1968), Jayme Neves (Neves et al. 1961), and Astolpho Ferraz de Siqueira (Barreto et al. 1963). These studies concluded the first phase of Brazilian protozoology. The co-authors of the works cited above certainly contributed to Brazilian protozoology and could be identified as founders of Brazilian protozoology.

The pioneers/founders, together with the students they supervised, nucleated the Brazilian protozoologist network. Thus, the environment favouring the construction of this field in Brazilian science was created. By supervising new students, organising scientific meetings and working side by side with agencies to create programmes for financial support, these scientists solidified the foundations of Brazilian protozoology and allowed the influx of new scientists into this area.

Immigration occurred in two waves. The entry of scientists between 1978-1993 might be a consequence of the creation of the Integrated Program for Endemic Diseases (PIDE), which was the Funding Programme from the Brazilian Council for Science Development (CNPq) that operated between 1976-1986. This programme invested the equivalent of 12 million American dollars in groups working in approximately 200 projects in endemic diseases (Gonçalves et al. 1988). Due to its differentiated financial policy, which means an initiative to influence the development of a special area, the programme had a huge impact on attracting more groups to work in protozoology, and our data reinforce this importance.

The second wave of incoming scientists occurred after 1998 and might be the consequence of the elevated number of fellowships and resources offered by the CNPq. In this sense, national politics favoured Brazilian science, and protozoology took advantage of this situation. However, we cannot forget the contribution of International Funding Programmes such as the Tropical Diseases Research (TDR) of the World Health Organization.

By tracking students who completed their $\mathrm{PhD}$ in protozoology, we observed that $85 \%$ of them were still involved with science. Considering that the other $15 \%$ might include people who teach in private universities using the knowledge acquired during their $\mathrm{PhD}$, we can conclude that the resources invested in protozoology were very well returned to society. However, it is time to reflect on whether the current number of students completing their PhDs will be harnessed in Brazil as has occurred in the past.

The scenario of protozoology in Brazil, as shown in Fig. 6, presents an increasing trend. Although the immigration dynamics presented in Fig. 5 showed a possible decrease in interest, Brazilian protozoology is the result of the work of pioneers and also (as evidenced by the green dots in Fig. 6) the consequence of the immigration of many scientists to protozoology who supervised their students in this area. It is important to reinforce that this flux requires financial support or strong funding programmes such as the PIDE and TDR.
Our goal in this communication was to show the dynamics of protozoologists and the impact of protozoology on Brazilian science. The same approach can be used to study contributions in other fields. The use of different criteria can group people in various ways to reveal other trees and identify other pioneers, even in protozoology. The genealogy presented here is one of multiple possible methods to track our past and hopefully point to our future.

From Isaac Newton to Stephen Hawking, the idea of "having seen further by standing on the shoulders of giants" has been used in science to recognise past mentors for new discoveries. We expect that in addition to serving as a source for research on the historical and paradigmatic aspects of Brazilian protozoology and positioning the contributions of the field to Brazilian science, this paper may be seen as a form of acknowledgement of the pioneering researchers who built the foundations of our work and inspired new generations of protozoology.

\section{ACKNOWLEDGEMENTS}

To Marcelo Reis, for grateful discussions concerning data analysis.

\section{REFERENCES}

Alves MJM, Colli W 1974. Agglutination of Trypanosoma cruzi by concavalin A. J Protozool 21: 575-578.

Andersen JP, Hammarfelt B 2011. Price revisited: on the growth of dissertations in eight research fields. Scientometrics 88: 371-383.

Barreto MP, Pessoa SB 1946. Contribuição para o estudo da distribuição geográfica dos flebótomos americanos (Diptera, Psychoidae). An Paul Med Cir 51: 261.

Barreto MP, Siqueira AF, Correa FM 1963. Study on reservoirs and forest vectors of Trypanosoma cruzi. I. Presence of Triatoma infestans (Hemiptera, Reduviidae) in forest outhouses. Rev Inst Med Trop Sao Paulo 5: 289-293.

Blackburn EH, Gall JG 1978. Tandemly repeated sequence at the termini of the extrachromosomal ribosomal RNA genes in Tetrahymena. J Mol Biol 120: 33-53.

Brener Z 1952. Complement fixation with L. brasiliensis in cutaneous American leishmaniasis and in Chagas disease. Hospital 41: 269-275.

Calkins GN 1911. The scope of protozoology. Science 34: 129-138.

Camargo EP 1964a. Growth and differantiation in Trypanosoma cru$z i$. I. Origin of metacyclic trypanosomas in liquid media. Rev Inst Med Trop Sao Paulo 6: 93-100.

Camargo ME 1964b. Improved technique of indirect immunofluorescence for serological diagnosis of toxoplasmosis. Rev Inst Med Trop Sao Paulo 6: 117-118.

Chagas C 1909. Nova tripanozomiaze humana. Estudos sobre a morfolojia e o ciclo evolutivo do Schizotrypanum cruzi n. gen., $n$. sp., ajente etiolojico de nova entidade morbida do homem. Mem Inst Oswaldo Cruz 1: 159-218.

Clayton CE 2002. Life without transcriptional control? From fly to man and back again. Embo J 21: 1881-1888.

Cordova KE, Furukawa H, Yaghi OM 2015. The development of global science. ACS Cent Sci 1: 18-23.

da Silva JR, de Paola D 1957. Histochemical changes in the hepatic reticuloendothelial system in kala-azar. Med Cir Farm 19: 211-215.

da Silva JR, Torres E 1957. Amebic abscess of the liver; review and case report. Rev Bras Gastroenterol 9: 215-234. 
de Brito T, Meira JA, Bassoi ON 1962. Contribution to the study of malaria. II. Pathology of the liver in acute malaria. Rev Inst Med Trop Sao Paulo 4: 105-111.

de Queiroz JM, Tafuri WL 1957. Lesion of the eye and adnexa in experimental schizotrypanosomiaisis in albino rats. Rev Assoc Med Bras 3: 256-262.

Deane LM, Deane MP 1954. Dogs naturally infected by Leishamnia donovani in Ceará. Hospital 45: 703-707.

Ferguson MA 1999. The structure, biosynthesis, and function of glycosylphosphatidylinositol anchors, and the contribution of trypanosome research. J Cell Sci 112: 2799-2809.

Ferguson MA, Haldar K, Cross GA 1985. Trypanosoma brucei variant surface glycoprotein has a sn-1,2-dimyristyl glycerol membrane anchor at its COOH terminus. J Biol Chem 260: 4963-4968.

Gonçalves A, Albuquerque RH, Lins MG, Neiva DS, Souza GF 1988. Avaliação exploratória de atuação bienal do Programa Integrado de Doenças Endêmicas do Conselho Nacional de Desenvolvimento Científico e Tecnológico PIDE/CNPq. Rev Inst Med Trop Sao Paulo 30: 109-117.

Heinzel FP, Sadick MD, Holaday BJ, Coffman RL, Locksley RM 1989. Reciprocal expression of interferon gamma of interleukin 4 during the resolution or progression of murine leishmaniasis. Evidence for expansion of distinct helper T-cell subsets. J Exp Med 169: 59-72.

Levenshtein VI 1966. Binary codes capable of correcting deletions, insertions, and reversals. Soviet Physics-Doklady 10: 707-710.

Martins AV, Brener Z, Mourão OG, Lima MM, de Souza MA, da Silva JE 1956. Autochthonous kala-azar in Minas Gerais. Rev Bras Malariol Doencas Trop 8: 555-563.

Mayer MG, Floeter-Winter LM 2012. Identification of SL addition trans-splicing acceptor sites in the internal trasncribed spacer I region of pre-rRNA in Leishmania (Leishmania) amazonensis. Mem Inst Oswaldo Cruz 107: 1070-1072.
Mena-Chalco JP, Rocha V 2014. Caracterização do banco de teses e dissertações da CAPES. In $4^{\circ}$ Encontro Brasileiro de Bibliometria e Cientometria, 14-16 de maio de 2014, Recife, 11 pp.

Meyer H, de Oliveira MX 1948. Cultivation of Trypanosoma cruzi in tissue cultures; a four day study. Parasitology 39: 91-94.

Moraes LCA, França EL, Pessoa RS, Fagundes DLG, Hernandes MG, Ribeiro VP, Gomes MA, Honório-França C 2015. The effect of INF-g and TGF-b in the functional activity of mononuclear cells in the presence of Entamoeba histolytica. Parasit Vectors 8: 413.

Neves J, Mayrink W, Mourao OG, Cardoso JP 1961. Kala-azar in Minas Gerais. Clinical and immuno-serological survey in the Hospital das Clínicas and in the Santa Casa de Misericórdia de Belo Horizonte, to identify cases of kala-azar. Hospital 59: 1165-1177.

Paraense WL 1952. Observations on a Brazilian strain of Plasmodium circumflexum. Mem Inst Oswaldo Cruz 50: 229-241.

Pessoa SB, Barreto MP 1945. Sobre a localização dos parasitos nos tecidos e a intensidade do parasitismo na leishamaniase tegumentar americana. Rev Paul Med 26: 355-357.

Prata A 1963. Treatment of kala-azar with amphotericin B. Trans $R$ Soc Trop Med Hyg 57: 266-268.

Roitman I 1969. Experiments in growing Trypanosoma conorhini on a synthetic medium. J Protozoology 16: 36.

Sampaio SA, Castro RM, Rivitti E, Sampaio SA 1971. Treatment of mucocutaneous (American) leishmaniasis with amphotericin B: report of 70 cases. Int J Dermatol 12: 388-394.

Shaw J, Lainson R 1968. Leishmaniasis in Brazil. II. Observation on enzootic leishamaniasis in the lower Amazonian Basin. Trans $R$ Soc Trop Med Hyg 62: 396-405.

Simpson AG, Stevens JR, Lukes J 2006. The evolution and diversity of kinetoplastid flagellates. Trends Parasitol 22: 168-174.

Sugimoto CR 2014. Academic genealogy. In B Cronin, CR Sugimoto (eds.), Beyond bibliometrics: harnessing multidimensional indicators of scholarly impact, MIT Press, Cambridge, p. 365-382. 
TABLE I

Descriptors used to screen theses on Coordination for the Improvement of Higher Education Personnel database

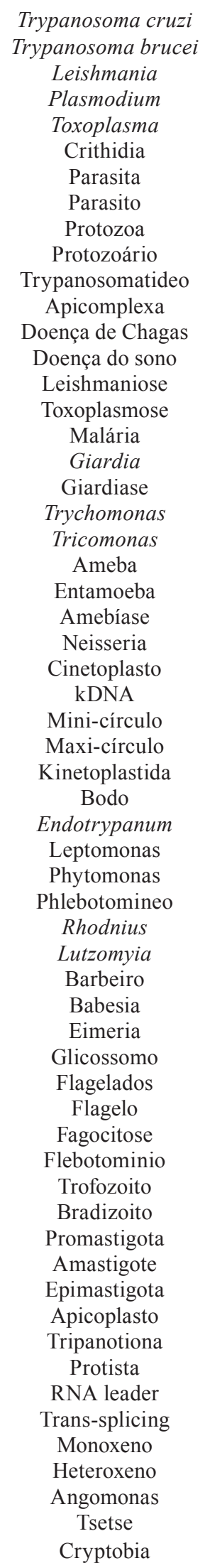


TABLE II

Scientists that supervised at least 10 theses/dissertations containing at least one of the 60 descriptors present on Supplementary Table I

Adauto Jose Goncalves De Araujo

Adevair Henrique Da Fonseca

Adjair Antonio Do Nascimento

Alda Maria Da Cruz

Aldina Maria Prado Barral

Alejandro Miguel Katzin

Alexandre Afranio Peixoto

Alexandre Barbosa Reis

Alfredo Miranda De Goes

Alvaro Jose Romanha

Alvimar Jose Da Costa

Ana Lucia Teles Rabello

Ana Maria De Castro

Ana Maria Jansen

Ana Paula Junqueira Kipnis

Andrea Caetano Da Silva

Andrea Mara Macedo

Angela Hampshire De Carvalho Santos Lopes

Angela Kaysel Cruz

Anita Hilda Straus Takahashi

Antonia Maria Ramos Franco

Antoniana Ursine Krettli

Antonio Luiz Pinho Ribeiro

Antonio Raimundo Lima Cruz Teixeira

Antonio Walter Ferreira

Armando De Oliveira Schubach

Arnaldo Zaha

Bartira Rossi Bergmann

Bianca Silvana Zingales

Bodo Wanke

Camila Indiani De Oliveira

Caris Maroni Nunes

Carlos Eduardo Tosta Da Silva

Carlos Graeff Teixeira

Carlos Luiz Massard

Carlos Renato Machado

Carlos Wilson Gomes Lopes

Celia Maria De Almeida Soares

Celia Maria Machado Barbosa De Castro

Celia Regina Da Silva Garcia

Celso Vataru Nakamura

Celuta Sales Alviano

Cezar Martins De Sa

Cirano Jose Ulhoa

Clara Lucia Barbieri Mestriner

Claude Pirmez

Claudia Ida Brodskyn

Claudia Maria Leal Bevilaqua

Claudia Martins Carneiro

Claudio Tadeu Daniel Ribeiro

Conceicao Ribeiro Da Silva Machado

Cristiana Ferreira Alves De Brito

Deborah Aparecida Negrao Correa

Denise Carmona Cara Machado

Dulciene Maria De Magalhaes Queiroz

Edelberto Santos Dias

Edesio Jose Tenorio De Melo

Edgar Marcelino Carvalho Filho

Edward Felix Silva

Eliane Lages Silva

Elisa Cupollilo

Supplementary data
Elizabeth Ferreira Rangel

Elizabeth Igne Ferreira

Elizabeth Malagueno De Santana

Elizabeth Spangler Andrade Moreira

Eloisa Amalia Vieira Ferro

Eloy Guillermo Castellon Bermudez

Elvio Carlos Moreira

Elvira Maria Saraiva Chequer Bou Habib

Erik Daemon De Souza Pinto

Erika Martins Braga

Eunice Aparecida Bianchi Galati

Evaldo Nascimento

Fernando Costa E Silva Filho

Fernando De Queiroz Cunha

Fernando Orefice

Flavio Antonio Pacheco De Araujo

Francisco Carlos Rodrigues De Oliveira

Francisco Juarez Ramalho Pinto

Gabriel Grimaldi Filho

George Alexandre Dos Reis

Geraldo Gileno De Sa Oliveira

Gervasio Henrique Bechara

Glaucius Oliva

Gloria Regina Franco

Guido Fontgalland Coelho Linhares

Guilherme Loureiro Werneck

Heitor Franco De Andrade Junior

Helene Santos Barbosa

Helio Langoni

Heloisa Werneck De Macedo

Hernando Antonio Del Portillo Obando

Hiro Goto

Ionice Felipe

Ises De Almeida Abrahamsohn

Italmar Teodorico Navarro

Jacenir Reis Dos Santos Mallet

Jaime Martins De Santana

Jeffrey Jon Shaw

Joao Aristeu Da Rosa

Joao Palermo Neto

Joao Santana Da Silva

Joaquim Hernan Patarroyo Salcedo

Jose Ciriaco Pinheiro

Jose Clovis Do Prado Junior

Jose Daniel Figueroa Villar

Jose Franco Da Silveira Filho

Jose Manuel Macario Rebelo

Jose Maria Alvarez Mosig

Jose Maria De Souza

Jose Maria Soares Barata

Jose Mauro Peralta

Jose Roberto Meyer Fernandes

Jose Roberto Mineo

Jose Rodrigues Coura

Joseli Lannes Vieira

Judith Kardos Klotzel

Julia Maria Costa Cruz

Juliana Lopes Rangel Fietto

Julio Scharfstein

Kenneth John Gollob

Lain Carlos Pontes De Carvalho 
Leda Quercia Vieira

Leonor Laura Pinto Leon

Leucio Camara Alves

Ligia Miranda Ferreira Borges

Lileia Goncalves Diotaiuti

Lucia Mendonca Previato

Lucile Maria Floeter Winter

Luis Carlos Crocco Afonso

Luis Eduardo Ramirez Giraldo

Luiz Carlos Alves

Luiz Hildebrando Pereira Da Silva

Luiz Ricardo Orsini Tosi

Manoel Barral Netto

Manoel Otavio Da Costa Rocha

Marcello Andre Barcinski

Marcelo Simao Ferreira

Marcia Cristina Paes

Marcia Dos Santos Lazera

Marco Aurelio Krieger

Marco Tulio Antonio Garciazapata

Marcos Andre Vannier Dos Santos

Maria Aparecida Da Gloria Faustino

Maria Aparecida Gomes

Maria Cristina Machado Motta

Maria Das Gracas Costa Alecrim

Maria De Fatima Ferreira Da Cruz

Maria De Fatima Martins Horta

Maria De Nazareth Silveira Leal De Meirelles

Maria Heloisa De Souza Lima Blotta

Maria Imaculada Muniz Barboza Junqueira

Maria Julia Manso Alves

Maria Julia Salim Pereira

Maria Lucia Belem Pinheiro

Maria Norma Melo

Maria Regina Reis Amendoeira

Maria Terezinha Bahia

Maria Valdrinez Campana Lonardoni

Mariangela Carneiro

Marinete Marins Povoa

Marlene Benchimol

Marlene Isabel Vargas Viloria

Marlene Tiduko Ueta

Marta De Lana

Marta Maria Geraldes Teixeira

Marta Tavares D Agosto

Mauricio Martins Rodrigues

Maurilio Jose Soares

Mauro Celio De Almeida Mazorchi

Monica Lucia Gomes

Nadia Regina Pereira Almosny

Nanci Do Nascimento

Nara Amelia Da Rosa Farias

Narcisa Leal Da Cunha E Silva

Nicolau Maues Da Serra Freire

Nobuko Yoshida

Octavio Fernandes Da Silva Filho

Olindo Assis Martins Filho

Osvaldo Pompilio De Melo Neto

Otavio Henrique Thiemann

Patricia De Azambuja Penna

Patricia Sampaio Tavares Veras

Paula Dias Bevilacqua

Paulo Eduardo Martins Ribolla
Paulo Filemon Paolucci Pimenta

Paulo Inacio Da Costa

Paulo Paes De Andrade

Pedro Lagerblad De Oliveira

Raimunda Nonata Ribeiro Sampaio

Regina Maria Barretto Cicarelli

Regina Maura Bueno Franco

Reginaldo Pecanha Brazil

Renata Guerra De Sa

Renato Arruda Mortara

Renato Augusto Da Matta

Ricardo Lourenco De Oliveira

Ricardo Luiz Dantas Machado

Ricardo Tostes Gazzinelli

Ricardo Wagner De Almeida Vitor

Rinaldo Aparecido Mota

Rita De Cassia Alves Alcantara De Menezes

Roberto Jose Da Silva Badaro

Rodrigo Correa De Oliveira

Romario Cerqueira Leite

Ronald Bastos Freire

Rosangela Zacarias Machado

Salvatore Giovanni De Simone

Samuel Goldenberg

Santuza Maria Ribeiro Teixeira

Selma Giorgio

Selma Maria Bezerra Jeronimo

Sergio Costa Oliveira

Sergio Danilo Junho Pena

Sergio De Albuquerque

Sergio Salles Xavier

Sergio Schenkman

Silvana Marques De Araujo

Silvia Reni Bortolin Uliana

Sinval Pinto Brandao Filho

Solange Lisboa De Castro

Solange Maria Gennari

Sonia Gumes Andrade

Stenio Perdigao Fragoso

Susie Andries Nogueira

Sylvio Celso Goncalves Da Costa

Tania Cremonini De Araujo Jorge

Tania Maria Valente Pacheco

Tecia Maria Ulisses De Carvalho

Teresa Cristina Bergamo Do Bomfim

Thais Cristina Baeta Soares Souto Padron

Thais Gomes Verzignassi Silveira

Ueslei Teodoro

Ulisses Gazos Lopes

Valeria Marcal Felix De Lima

Vanderlei Rodrigues

Vanete Thomaz Soccol

Vanize De Oliveira Macedo

Vicente De Paula Antunes Teixeira

Virmondes Rodrigues Junior

Wagner Luiz Tafuri

Walderez Ornelas Dutra

Wanderley De Souza

Wanderli Pedro Tadei

Washington Luiz Tafuri

Wilson Mayrink

Yara De Miranda Gomes

Yara Maria Traub Cseko

source: Coordination for the Improvement of Higher Education Personnel database in the period from 1987-2011. 
TABLE III

Classification of names according to diagram in Fig. 3A

\begin{tabular}{|c|c|c|c|}
\hline Name & Group & Name & Group \\
\hline Absalom Lima Filgueira & $\mathrm{c}$ & Alexandre Morrot Lima & a \\
\hline Ada Amalia Ayala Urdapilleta & $\mathrm{d}$ & Alexandre Nizio Maria & $\mathrm{c}$ \\
\hline Adalberto Ramon Vieyra & $\mathrm{c}$ & Alexandre Rego Souza Pinto & - \\
\hline Adauto Castelo & $\mathrm{c}$ & Alfredo Carlos Rodrigues de Azevedo & $\mathrm{a}$ \\
\hline Adauto Jose Goncalves de Araujo & $\mathrm{c}$ & Alfredo Jose Afonso Barbosa & $\mathrm{c}$ \\
\hline Adelina Diaz de Ramirez & - & Alfredo Jose Altamirano Enciso & a \\
\hline Ademir de Jesus Martins Junior & $\mathrm{c}$ & Alfredo Miranda de Goes & $\mathrm{c}$ \\
\hline Aderson Zottis & e & Alice Machado da Silva & e \\
\hline Adevair Henrique da Fonseca & $\mathrm{c}$ & Alicia Kompalic Cristo & - \\
\hline Adjair Antonio do Nascimento & $\mathrm{c}$ & Aline Aparecida Rezende Rodrigues & $\mathrm{c}$ \\
\hline Adrian Martin Pohlit & a & Aline Cardoso Caseca Volotao & a \\
\hline Adriana Barrinha Fernnades & $\mathrm{a}$ & Aline Chaves Alexandrino & $\mathrm{a}$ \\
\hline Adriana Carlos Rodrigues Fuzato & $\mathrm{d}$ & Aline de Azevedo & e \\
\hline Adriana da Roza Chaves & $\mathrm{c}$ & Aline do Carmo Franca Botelho & $\mathrm{c}$ \\
\hline Adriana Degrossoli & e & Aline Fernandes Angella Martins & - \\
\hline Adriana dos Passos Lemos & $\mathrm{e}$ & Allan Kardec Ribeiro Galardo & $\mathrm{a}$ \\
\hline Adriana Goncalves de Oliveira & $\mathrm{c}$ & Aluizio Rosa Prata & a \\
\hline Adriana Jardim de Almeida & a & Alvaro Augusto Ribeiro D Almeida Couto & $\mathrm{a}$ \\
\hline Adriana Lanfredi Rangel & $\mathrm{d}$ & Alvaro Cantini Nunes & e \\
\hline Adriana Melo Ferreira & $\mathrm{d}$ & Alvaro Eduardo Eiras & $\mathrm{c}$ \\
\hline Adriana Oliveira Costa & $\mathrm{a}$ & Alvaro Fernando Acosta Serrano & $\mathrm{b}$ \\
\hline Adriana Oliveira dos Santos & a & Alvaro Jose Romanha & $\mathrm{a}$ \\
\hline Adriane Pimenta da Costa Val Bicalho & a & Alverne Passos Barbosa & $\mathrm{d}$ \\
\hline Adriane Regina Todeschini & $\mathrm{a}$ & Alvimar Jose da Costa & $\mathrm{c}$ \\
\hline Adriano Defini Andricopulo & $\mathrm{a}$ & Amadeu Cury & - \\
\hline Adriano Gomes da Silva & $\mathrm{a}$ & Amanda Fortes Francisco & $\mathrm{a}$ \\
\hline Alan Mitchell Durham & $\mathrm{c}$ & America Kaori Tanaka & - \\
\hline Alan Sher & $\mathrm{b}$ & Amilcar Vianna Martins & a \\
\hline Alba Valeria Machado da Silva & a & Ana Acacia de Sa Pinheiro & a \\
\hline Albert Thomas Londero & - & Ana Carolina Borella Anhe & e \\
\hline Alberto Carvalho da Silva & - & Ana Carolina Bussacos Maranhao & d \\
\hline Alberto Francisco Fachado Carvajales & - & Ana Carolina Campi Azevedo & a \\
\hline Alberto Gomes Ferreira & $\mathrm{c}$ & Ana Carolina de Aguiar Vasconcelos Carneiro & $\mathrm{a}$ \\
\hline Alberto Jesus Cornejo Mora & - & Ana Carolina dos Santos Monteiro & e \\
\hline Alcides Milton da Silva & $\mathrm{d}$ & Ana Carolina Fonseca Lindoso Melo & $\mathrm{d}$ \\
\hline Alda Maria da Cruz & $\mathrm{a}$ & Ana Carolina Paulo Vicente & $\mathrm{c}$ \\
\hline Alda Maria Teixeira Ferreira & a & Ana Carolina Renno Sodero & $\mathrm{c}$ \\
\hline Aldina Maria Prado Barral & $\mathrm{a}$ & Ana Claudia Goncalves & $\mathrm{c}$ \\
\hline Aldo Mellender de Araujo & $\mathrm{c}$ & Ana Claudia Ribeiro e Silva & - \\
\hline Alejandra Saori Araki & $\mathrm{a}$ & Ana Cristina Arend & - \\
\hline Alejandro Miguel Katzin & a & Ana Cristina Bahia Nascimento & a \\
\hline Alena Mayo Iniguez & - & Ana Cristina da Silva Pinto & a \\
\hline Alessandra Conceicao Faria Aguiar Campos & $\mathrm{c}$ & Ana Cristina de Carvalho Botelho & a \\
\hline Alessandra D Almeida Filardy & $\mathrm{a}$ & Ana Cristina dos Santos Otero & $\mathrm{d}$ \\
\hline Alessandra Gutierrez Oliveira & a & Ana Cristina Rodrigues Saldanha & $\mathrm{c}$ \\
\hline Alessandra Loureiro Morassutti & $\mathrm{c}$ & Ana Cristina Vianna Mariano da Rocha Lima & a \\
\hline Alessandra Mara Alves Ragozo & $\mathrm{a}$ & Ana de Cassia Oliveira Rosa Vexenat & - \\
\hline Alessandra Rejane Ericsson de Oliveira & e & Ana de Nazare Martins da Silva & e \\
\hline Alessandra Santos D Alencar & $\mathrm{c}$ & Ana Iara da Costa Ferreira & $\mathrm{d}$ \\
\hline Alessandra Scofield Amaral & a & Ana Leuch Lozovei & $\mathrm{d}$ \\
\hline Alessandro Aparecido Rodrigues da Silva & a & Ana Lucia Abreu Silva & $\mathrm{a}$ \\
\hline Alessandro Comaru Pasqualotto & $\mathrm{c}$ & Ana Lucia Teles Rabello & a \\
\hline Alex Garcia Todorov & $\mathrm{d}$ & Ana Maria Barral de Martinez & $\mathrm{c}$ \\
\hline Alexander Sibajev & a & Ana Maria Caetano de Faria & $\mathrm{c}$ \\
\hline Alexandra Lena Galetovic Carabantes & - & Ana Maria de Castro & a \\
\hline Alexandre Afranio Peixoto & a & Ana Maria Dieckmann & e \\
\hline Alexandre Barbosa Reis & a & Ana Maria Jansen Franken & $\mathrm{a}$ \\
\hline
\end{tabular}




\begin{tabular}{|c|c|c|c|}
\hline Name & Group & Name & Group \\
\hline Ana Maria Reis Ferreira & $\mathrm{c}$ & Angela Hampshire de Carvalho Santos Lopes & $\mathrm{a}$ \\
\hline Ana Maria Revoredo da Silva Ventura & $\mathrm{a}$ & Angela Kaysel Cruz & $\mathrm{a}$ \\
\hline Ana Paula Cabral de Araujo Lima & a & Anibal Antonio da Silva Pereira & a \\
\hline Ana Paula Campanelli & $\mathrm{e}$ & Anissa Daliry & e \\
\hline Ana Paula dos Reis Velloso Siciliano & $\mathrm{d}$ & Anita Hilda Straus Takahashi & $\mathrm{a}$ \\
\hline Ana Paula Ferreira Lopes Correa & e & Annamaria Ravara Vago & a \\
\hline Ana Paula Galvao da Silva & $\mathrm{d}$ & Anselmo Afonso Golynski & e \\
\hline Ana Paula Iglesias Santin & $\mathrm{c}$ & Antonia Maria Ramos Franco Pereira & a \\
\hline Ana Paula Junqueira Kipnis & $\mathrm{c}$ & Antoniana Ursine Krettli & a \\
\hline Ana Paula Maia Peixoto Marino & a & Antonio Campos Neto & $\mathrm{c}$ \\
\hline Ana Paula Moreira & $\mathrm{e}$ & Antonio Carlos Alves Meirelles Filho & - \\
\hline Ana Paula Peconick & $\mathrm{c}$ & Antonio Carlos Francesconi do Valle & $\mathrm{c}$ \\
\hline Ana Paula Pimentel Cassilhas & $\mathrm{d}$ & Antonio Cesar Rios Leite & $\mathrm{c}$ \\
\hline Ana Paula Rocha Gadelha & $\mathrm{a}$ & Antonio Cezar Rocha Cavalcante & $\mathrm{d}$ \\
\hline Ana Paula Salles Moura Fernandes & $\mathrm{a}$ & Antonio de Deus Filho & $\mathrm{c}$ \\
\hline Ana Silvia Dagnone & $\mathrm{c}$ & Antonio de Oliveira Lima & $\mathrm{a}$ \\
\hline Ana Tereza de Mendonca Viveiros & $\mathrm{c}$ & Antonio Florencio De Figueiredo & a \\
\hline Ana Yece das Neves Pinto & a & Antonio Gildo de Bianchi & $\mathrm{c}$ \\
\hline Analina Furtado Valadao & $\mathrm{c}$ & Antonio Lima Cruz Teixeira & a \\
\hline Anamaria Aranha Camargo & e & Antonio Lucio Teixeira Junior & $\mathrm{c}$ \\
\hline Anamaria Mello Miranda Paniago & $\mathrm{c}$ & Antonio Luiz Pinho Ribeiro & $\mathrm{c}$ \\
\hline Anderson Barbosa de Moura & a & Antonio Marcos Guimaraes & $\mathrm{a}$ \\
\hline Anderson Sena Barnabe & $\mathrm{a}$ & Antonio Medeiros Peregrino da Silva & $\mathrm{c}$ \\
\hline Andre Dreyfus & $\mathrm{c}$ & Antonio Neres Norberg & $\mathrm{c}$ \\
\hline Andre Flavio Soares Ferreira Rodrigues & $\mathrm{c}$ & Antonio Paes de Carvalho & $\mathrm{c}$ \\
\hline Andre Gustavo Tempone Cardoso & a & Antonio Paulino Ribeiro Sobrinho & $\mathrm{c}$ \\
\hline Andre Luis Bombeiro & e & Antonio Sesso & $\mathrm{c}$ \\
\hline Andre Luiz Fonseca de Souza & $\mathrm{a}$ & Antonio Walter Ferreira & $\mathrm{a}$ \\
\hline Andre Luiz Jeovanio da Silva & $\mathrm{d}$ & Aparecida de Fatima Michelin & $\mathrm{a}$ \\
\hline Andre Luiz Land Curi & $\mathrm{c}$ & Aramis Augusto Pinto & $\mathrm{c}$ \\
\hline Andre Luiz Pedrosa & a & Argemiro Sanavria & $\mathrm{c}$ \\
\hline Andre Luiz Rodrigues Roque & $\mathrm{a}$ & Ariane Baratta Masini & e \\
\hline Andre Nobrega Pitaluga & a & Ariane Machado Lima & $\mathrm{e}$ \\
\hline Andrea Alice da Silva & a & Aristarco Goncalves Siqueira Filho & $\mathrm{c}$ \\
\hline Andrea Aparecida Morais Fernandes & $\mathrm{a}$ & Aristeu Vieira da Silva & $\mathrm{c}$ \\
\hline Andrea Bouer & a & Aristides Cheto de Queiroz & - \\
\hline Andrea Caetano da Silva & $\mathrm{a}$ & Arlei Marcili & $\mathrm{a}$ \\
\hline Andrea Carla Leite Chaves & a & Arlene de Jesus Mendes Caldas & $\mathrm{a}$ \\
\hline Andrea Cristina Higa Nakaghi & $\mathrm{c}$ & Arlete Aparecida Martins Coelho Castelo & $\mathrm{c}$ \\
\hline Andrea Cristina Veto Arnholdt & a & Arlie C Todd & - \\
\hline Andrea Henriques Pons & a & Armando de Oliveira Schubach & $\mathrm{a}$ \\
\hline Andrea Mara Macedo & a & Arnaldo FI da Rocha & - \\
\hline Andrea Pereira de Souza & $\mathrm{a}$ & Arnaldo Zaha & $\mathrm{c}$ \\
\hline Andrea Rodrigues Avila & a & Artur Torres Cordeiro & $\mathrm{a}$ \\
\hline Andrea Silvestre de Sousa & $\mathrm{c}$ & Astolpho Ferraz de Siqueira & a \\
\hline Andrea Sobral de Almeida & a & Augusto Mello Simoes Barbosa & $\mathrm{a}$ \\
\hline Andrea Teixeira de Carvalho & a & Aurelio Pedroso Junior & e \\
\hline Andrea Vieira Goncalves & $\mathrm{d}$ & Aurelio Vicente Graca de Souza & e \\
\hline Andreia Dantas Medeiros & $\mathrm{c}$ & Aurilene Monteiro Bandeira & $\mathrm{c}$ \\
\hline Andreia Pires Dantas & $\mathrm{e}$ & Avelino Jose Bittencourt & $\mathrm{c}$ \\
\hline Andres Jimenez Galisteo Junior & a & Barbara Cruz Tavares de Macedo Fernandes & $\mathrm{a}$ \\
\hline Andrew John George Simpson & $\mathrm{b}$ & Barbara Kellen Antunes Borges & $\mathrm{d}$ \\
\hline Andrey Jose de Andrade & a & Bartira Rossi Bergmann & $\mathrm{a}$ \\
\hline Andreza Pain Marcelino & a & Beatriz Dolabela de Lima & a \\
\hline Anette Kelsei Partata & $\mathrm{d}$ & Beatriz Gomes Brazil & $\mathrm{a}$ \\
\hline Angela Andrade Maestrini & - & Beatriz Gomes Guimaraes & a \\
\hline Angela Cardoso de Alvarenga & $\mathrm{e}$ & Beatriz Lilian da Silva Costa Souza & $\mathrm{a}$ \\
\hline Angela Cristina Verissimo Junqueira & a & Beatriz Rossetti Ferreira & a \\
\hline Angela Cristina Volpini & a & Bellisa de Freitas Barbosa & - \\
\hline
\end{tabular}




\begin{tabular}{|c|c|c|c|}
\hline Name & Group & Name & Group \\
\hline Bernardo Galvao Castro & a & Carlos Eugenio Cavasini & $\mathrm{a}$ \\
\hline Bernardo Simoes Franklin & e & Carlos Frederico Loiola & $\mathrm{b}$ \\
\hline Bernd Liess & - & Carlos Frederico Martins Menck & $\mathrm{c}$ \\
\hline Betania Maria Ribeiro & $\mathrm{e}$ & Carlos Graeff Teixeira & $\mathrm{c}$ \\
\hline Bianca Brand Ederli & $\mathrm{a}$ & Carlos Gustavo Regis da Silva & $\mathrm{a}$ \\
\hline Bianca Chiganer Cramer Ribeiro & - & Carlos Henryque De Souza E Silva & - \\
\hline Bianca Ervatti Gama & $\mathrm{e}$ & Carlos Jose de Carvalho Pinto & $\mathrm{a}$ \\
\hline Bianca Perdigao Olivieri & a & Carlos Luiz Massard & a \\
\hline Bianca Silvana Zingales & $\mathrm{a}$ & Carlos Massayuki Kikuti & $\mathrm{e}$ \\
\hline Blima Fux & $\mathrm{a}$ & Carlos Mauricio de Figueiredo Antunes & $\mathrm{a}$ \\
\hline Bodo Wanke & $\mathrm{c}$ & Carlos Medicis Morel & a \\
\hline Braulio Muzzi Ribeiro de Oliveira & - & Carlos Noriyuki Kaneto & $\mathrm{a}$ \\
\hline Bruna Cunha Gondim de Alencar & - & Carlos Ramon Ruiz Miranda & - \\
\hline Bruna Guimaraes Oliveira & - & Carlos Renato Machado & $\mathrm{a}$ \\
\hline Bruno Antonio Marinho Sanchez & a & Carlos Roberto Alves & - \\
\hline Bruno Augusto Maciel Guedes & $\mathrm{c}$ & Carlos Wilson Gomes Lopes & $\mathrm{a}$ \\
\hline Bruno da Rocha Azevedo & $\mathrm{a}$ & Carmen Dea Ribeiro Paula & $\mathrm{c}$ \\
\hline Bruno de Bezerril Andrade & a & Carol Homewood & $\mathrm{b}$ \\
\hline Bruno Luiz Fonseca Schamber Reis & $\mathrm{e}$ & Carolina Borsoi Moraes & $\mathrm{a}$ \\
\hline Bruno Pereira Berto & a & Carolina de Oliveira Mendes Aguiar & $\mathrm{a}$ \\
\hline Byanca Regina de Paiva & $\mathrm{d}$ & Carolina Furtado Torres da Silva & a \\
\hline Caio Benjamin Dias & $\mathrm{c}$ & Carolina Guilherme Prestes Beyrodt & $\mathrm{a}$ \\
\hline Camila Alves Bandeira Falcao & $\mathrm{d}$ & Carolina Maria Vianna de Freitas & $\mathrm{c}$ \\
\hline Camila Augusta de Oliveira Martins & $\mathrm{a}$ & Carolina Nascimento Spiegel & $\mathrm{a}$ \\
\hline Camila del Sarto Macedo & - & Caroline Dantas de Oliveira & a \\
\hline Camila Indiani de Oliveira & a & Caroline Furtado Junqueira & - \\
\hline Camila Junqueira Mazzoni & e & Cassia Abadia Xavier Costa & $\mathrm{d}$ \\
\hline Camila Maciel de Sousa & $\mathrm{c}$ & Cassio da Silva Baptista & $\mathrm{d}$ \\
\hline Camila Marques Adade & a & Catarina Gadelha & a \\
\hline Camila Sachelli Ramos & - & Catia Amancio Almeida & $\mathrm{c}$ \\
\hline Camila Souza Lemos & $\mathrm{d}$ & Cecilia Beatriz Fiuza Favali & $\mathrm{a}$ \\
\hline Camilo Adalton Mariano da Silva & $\mathrm{c}$ & Ceclie Maria Antunes B Biancardi & a \\
\hline Candida Fagundes Teixeira & $\mathrm{c}$ & Celene Maria de Oliveira Simoes Alves & $\mathrm{a}$ \\
\hline Cardi Bruno Andrade & $\mathrm{c}$ & Celeste da Silva Freitas de Souza & a \\
\hline Carina Elisei de Oliveira & $\mathrm{a}$ & Celeste Fava Netto & - \\
\hline Carina Margonari de Souza & a & Celia Alves de Almeida & - \\
\hline Caris Maroni Nunes & $\mathrm{a}$ & Celia Aparecida Paulino & $\mathrm{c}$ \\
\hline Carl Peter Von Dietrich & $\mathrm{c}$ & Celia Maria de Almeida Soares & $\mathrm{c}$ \\
\hline Carla Claser & $\mathrm{d}$ & Celia Maria Gontijo Ferreira & a \\
\hline Carla Cristina Braz Louly & $\mathrm{c}$ & Celia Maria Machado Barbosa de Castro & $\mathrm{c}$ \\
\hline Carla de Almeida Cardozo & - & Celia Maria Silva Pedrosa & $\mathrm{a}$ \\
\hline Carla Domingues dos Santos & $\mathrm{a}$ & Celia Maria Vieira Vendrame & $\mathrm{a}$ \\
\hline Carla Gasparotto Shande Vasconcelos & - & Celia Regina da Silva Garcia & a \\
\hline Carla Maria de Souza Menezes & $\mathrm{c}$ & Celio Geraldo Freire de Lima & $\mathrm{a}$ \\
\hline Carla Monica Pinheiro & a & Celio Lopes Silva & $\mathrm{c}$ \\
\hline Carlos Alberto do Nascimento Ramos & $\mathrm{e}$ & Celso Barbosa de Santanna Filho & $\mathrm{e}$ \\
\hline Carlos Alberto Garofalo & $\mathrm{c}$ & Celso Cruz Tavares & a \\
\hline Carlos Alberto Pereira Tavares & $\mathrm{a}$ & Celso Martins Pinto & $\mathrm{e}$ \\
\hline Carlos Alexandre de Amorim Garcia & $\mathrm{c}$ & Celso Vataru Nakamura & a \\
\hline Carlos Capistrano Goncalves de Oliveira & a & Celuta Sales Alviano & a \\
\hline Carlos Chagas & $\mathrm{a}$ & Cezar Martins de Sa & $\mathrm{a}$ \\
\hline Carlos da Silva Lacaz & $\mathrm{c}$ & Charalambos P Kyriacou & $\mathrm{c}$ \\
\hline Carlos Eduardo Almeida & $\mathrm{a}$ & Charles Alfredo Covarrubias Flores & - \\
\hline Carlos Eduardo Calzavara Silva & $\mathrm{c}$ & Charles Anacleto & $\mathrm{c}$ \\
\hline Carlos Eduardo Maia Gomes & $\mathrm{a}$ & Charles Keneth Whitehair & - \\
\hline Carlos Eduardo Pereira Corbett & a & Charles Salmon & - \\
\hline Carlos Eduardo Peres Sampaio & $\mathrm{e}$ & Christian Macagnan Probst & a \\
\hline Carlos Eduardo Tadokoro & a & Christian Robson de Souza Reis & $\mathrm{c}$ \\
\hline Carlos Eduardo Tosta da Silva & a & Christiane Contigli & $\mathrm{c}$ \\
\hline
\end{tabular}




\begin{tabular}{|c|c|c|c|}
\hline Name & Group & Name & Group \\
\hline Christina Alves Peixoto & $\mathrm{c}$ & Cristiane Divan Baldani & $\mathrm{d}$ \\
\hline Chung Man Chin & $\mathrm{a}$ & Cristiane Gaboggini Melo de Pinheiro & - \\
\hline Cibele Baptista & $d$ & Cristiane Guimaraes Morais & $\mathrm{d}$ \\
\hline Cicero Brasileiro Mello Neto & $\mathrm{a}$ & Cristiane Varella Lisboa & $\mathrm{a}$ \\
\hline Cid Sergio Ferreira & $\mathrm{c}$ & Cristiano Barros de Melo & $\mathrm{e}$ \\
\hline Cinthia Furst Leroy Gomes & a & Cristiano Marcelo Espinola Carvalho & $\mathrm{e}$ \\
\hline Cintia Aparecida de Jesus Pereira & $\mathrm{c}$ & Cristina de Oliveira Massoco & $\mathrm{c}$ \\
\hline Cintia Fernandes de Souza & $\mathrm{a}$ & Cristina de Souza Chaves & $\mathrm{a}$ \\
\hline Cintia Fontes Alves & $\mathrm{d}$ & Cristina Germani Fialho & $\mathrm{d}$ \\
\hline Cirano Jose Ulhoa & $\mathrm{c}$ & Cristina Henriques & $\mathrm{a}$ \\
\hline Clara Lucia Barbieri Mestriner & a & Cristina Ribeiro de Barros Cardoso & $\mathrm{c}$ \\
\hline Clarence A Speer & $\mathrm{c}$ & Cristina Toscano Fonseca & $\mathrm{c}$ \\
\hline Clarissa Romero Teixeira & a & Cristina Wide Pissetti & $\mathrm{a}$ \\
\hline Claude Andre Solari & $\mathrm{c}$ & Cristine Hirsch Monteiro & $\mathrm{c}$ \\
\hline Claude Pirmez & a & Crodowaldo Pavan & $\mathrm{c}$ \\
\hline Claudia Alves de Andrade Coelho & a & Cynthia Rayol de Andrade & - \\
\hline Claudia Cristina Gulias Gomes & $\mathrm{c}$ & Daniel Adesse Pedra Martins & - \\
\hline Claudia de Matos Raja Gabaglia & $\mathrm{d}$ & Daniel Claudio de Oliveira Gomes & a \\
\hline Claudia Dias Zettermann & $\mathrm{c}$ & Daniel Sa Carvalho & $\mathrm{d}$ \\
\hline Claudia Ida Brodskyn de Assis & a & Daniel Vieira Dias & $\mathrm{d}$ \\
\hline Claudia Magalhaes Calvet & a & Daniela de Melo Resende & a \\
\hline Claudia Maia Brigagao & a & Daniela de Oliveira Procopio & $\mathrm{d}$ \\
\hline Claudia Mara Lara Melo Coutinho & $\mathrm{a}$ & Daniela de Pita Pereira & a \\
\hline Claudia Maria Leal Bevilaqua & a & Daniela de Stefani Marquez & $\mathrm{a}$ \\
\hline Claudia Maria Valete Rosalino & $\mathrm{c}$ & Daniela Fiori Gradia & $\mathrm{a}$ \\
\hline Claudia Martins Carneiro & $\mathrm{a}$ & Daniela Goncales Rando & $\mathrm{c}$ \\
\hline Claudia Moura de Melo & $\mathrm{c}$ & Daniela Lelles de Souza & - \\
\hline Claudia Portes Santos Silva & $\mathrm{d}$ & Daniela Luz Ambrosio & $\mathrm{a}$ \\
\hline Claudia Regina Lopes Cardoso & $\mathrm{c}$ & Daniela Parada Pavoni & $\mathrm{a}$ \\
\hline Claudia Soares Santos Lessa & $\mathrm{c}$ & Daniela Pedrassani & $\mathrm{e}$ \\
\hline Claudia Soares Zouain & $\mathrm{c}$ & Daniela Rodrigues de Faria & a \\
\hline Claudia Sossai Soares & $\mathrm{c}$ & Daniela Sales Alviano Moreno & $\mathrm{c}$ \\
\hline Claudia Torres Codeco & $\mathrm{c}$ & Daniela Santoro Rosa & $\mathrm{e}$ \\
\hline Claudia Zuleida Gonzalez Lombana & $\mathrm{d}$ & Daniele da Silva Ferreira & $\mathrm{d}$ \\
\hline Claudine Herzlich & - & Daniele dos Santos Andrade & $\mathrm{a}$ \\
\hline Claudiney Melquiades Rodrigues & e & Daniele Pereira de Castro & $\mathrm{a}$ \\
\hline Claudio Alessandro Massamitsu Sakamoto & $\mathrm{c}$ & Daniella Alchaar D Avila & $\mathrm{a}$ \\
\hline Claudio Baptista de Carvalho & $\mathrm{c}$ & Daniella Areas Mendes da Cruz & $\mathrm{e}$ \\
\hline Claudio Osmar Pereira Alexandre & e & Daniella Castanheira Bartholomeu & $\mathrm{a}$ \\
\hline Claudio Roberto Madruga & a & Daniella Regina Arantes Martins & $\mathrm{a}$ \\
\hline Claudio Romero Farias Marinho & $\mathrm{a}$ & Danielle Ferreira de Magalhaes & $\mathrm{a}$ \\
\hline Claudio Tadeu Daniel Ribeiro & $\mathrm{a}$ & Danielle Frias Ribeiro Bisaggio & $\mathrm{c}$ \\
\hline Claudio Vieira da Silva & a & Danielle Gomes Passos Silva & $\mathrm{e}$ \\
\hline Clayton Luiz Borges & $\mathrm{c}$ & Danielle Maria Nascimento Moura & $\mathrm{a}$ \\
\hline Clea Andrade Chiari & a & Danielle Pereira Cavalcanti & $\mathrm{a}$ \\
\hline Clea Nazare Carneiro Bichara & $\mathrm{a}$ & Danielle Pereira Vieira & a \\
\hline Cleber Oliveira Soares & e & Danielle Reis Napolitano & $\mathrm{a}$ \\
\hline Cleudson Nery de Castro & $\mathrm{c}$ & Danilo Ciccone Miguel & $\mathrm{a}$ \\
\hline Cleusa Alves Theodoro Rodrigues & a & Darcy Fontoura de Almeida & $\mathrm{c}$ \\
\hline Clever Gomes Cardoso & a & Dario Abbud Righi & $\mathrm{c}$ \\
\hline Cloe Duarte Fernandes & $\mathrm{d}$ & David Charles Warhurst & $\mathrm{b}$ \\
\hline Colete Fonseca & a & David Eduardo Barroso & $\mathrm{c}$ \\
\hline Conceicao Ribeiro da Silva Machado & a & David Nascimento Silva Teixeira & $\mathrm{c}$ \\
\hline Constanca Felicia de Paoli de Carvalho Britto & a & Debora Barreiros Petropolis & $\mathrm{d}$ \\
\hline Cornelius Bernard Van Niel & - & Debora de Oliveira Lopes & $\mathrm{c}$ \\
\hline Cristiana Ferreira Alves de Brito & a & Debora Naves Santos & $\mathrm{c}$ \\
\hline Cristiane Alves da Silva Menezes & - & Debora Pereira Garcia Melo & $\mathrm{a}$ \\
\hline Cristiane da Silva Stabenow & $\mathrm{c}$ & Debora Rochelly Alves Ferreira & $\mathrm{e}$ \\
\hline Cristiane de Souza Carvalho & $\mathrm{d}$ & Debora Rose de Oliveira & $\mathrm{e}$ \\
\hline
\end{tabular}




\begin{tabular}{|c|c|c|c|}
\hline Name & Group & Name & Group \\
\hline Deboraci Brito Prates & a & Eduardo Osorio Cisalpino & - \\
\hline Deborah Aparecida Negrao Correa & $\mathrm{c}$ & Eduardo Penna Franca & - \\
\hline Deila Jordao Franco & $\mathrm{d}$ & Eduardo Sergio da Silva & $\mathrm{a}$ \\
\hline Deise Aparecida de Oliveira Silva & a & Edward Felix Silva & $\mathrm{a}$ \\
\hline Deivid Costa Soares & a & Edwards Frazao Teixeira & a \\
\hline Delir Correa Gomes Maues da Serra Freire & $\mathrm{c}$ & Egler Chiari & a \\
\hline Denise Barcante Castro Pinto & a & Elaine Bernardo Mapeli & $\mathrm{c}$ \\
\hline Denise Bertulucci Rocha Rodrigues & a & Elaine Soares Coimbra & a \\
\hline Denise Borges dos Santos Dias & a & Elaine Speziali de Faria & $\mathrm{c}$ \\
\hline Denise Carmona Cara Machado & $\mathrm{c}$ & Elen Mello de Souza & $\mathrm{e}$ \\
\hline Denise Costa Arruda & e & Elenice Moreira Lemos & a \\
\hline Denise da Silveira Lemos & a & Eleuza Rodrigues Machado & $\mathrm{c}$ \\
\hline Denise Fonseca Cortes & a & Eliame Mouta Confort & a \\
\hline Denise Oliveira da Rosa & $\mathrm{d}$ & Eliana Lucia Tomaz do Nascimento & $\mathrm{a}$ \\
\hline Denise Valle & $\mathrm{a}$ & Eliane Correa de Santana & - \\
\hline Diamar da Costa Pinto & $\mathrm{d}$ & Eliane Cristina Laurentino & $\mathrm{d}$ \\
\hline Diana Cordeiro Taboada & $\mathrm{c}$ & Eliane de Morais Teixeira & a \\
\hline Diana Magalhaes de Oliveira & $\mathrm{c}$ & Eliane Gomes de Almeida & $\mathrm{d}$ \\
\hline Diane Mcmahon Pratt & $\mathrm{b}$ & Eliane Lages Silva & $\mathrm{a}$ \\
\hline Diego Peres Alonso & a & Eliane Maria Vieira Milward De Azevedo & $\mathrm{c}$ \\
\hline Dilvia Ferreira da Silva & $\mathrm{a}$ & Eliane Perlatto Moura & $\mathrm{a}$ \\
\hline Diogenes Aparicio Garcia Cortez & $\mathrm{c}$ & Elias Jorge Facury Filho & $\mathrm{c}$ \\
\hline Dirceu Bartolomeu Greco & $\mathrm{c}$ & Eliete Cavalcante da Silva & - \\
\hline Dirceu Joaquim Costa & $\mathrm{e}$ & Elieth Afonso de Mesquita & a \\
\hline Diva do Carmo Teixeira & $\mathrm{c}$ & Eline S Prado & $\mathrm{c}$ \\
\hline Djair dos Santos de Lima E Souza & $\mathrm{c}$ & Eliomara Sousa Sobral Alves & e \\
\hline Donaldo Rosa Pires Junior & $\mathrm{c}$ & Elisa Cupolillo & a \\
\hline Dong Hyun Kim & - & Elisa Neves Vianna & $\mathrm{a}$ \\
\hline Dorlene Maria Cardoso Aquino & $\mathrm{c}$ & Elisa San Martin Mouriz Savani & $\mathrm{a}$ \\
\hline Dorly de Freitas Buchi & $\mathrm{c}$ & Elisabeth Neumann & $\mathrm{c}$ \\
\hline Duarte da Silva & $\mathrm{c}$ & Elisaldo Luiz de Araujo Carlini & $\mathrm{c}$ \\
\hline Dulce Helena Ferreira De Souza & $\mathrm{a}$ & Elisandra Marcia Rodrigues & e \\
\hline Dulciene Maria de Magalhaes Queiroz & $\mathrm{c}$ & Elita Scio Fontes & $\mathrm{c}$ \\
\hline Dyann Wirth & $\mathrm{b}$ & Elizabeth Castro Moreno & $\mathrm{a}$ \\
\hline Ed Palmer & - & Elizabeth Cristina de Almeida Bessa & - \\
\hline Edalton dos Reis Silva & $\mathrm{c}$ & Elizabeth de Souza Neves & $\mathrm{a}$ \\
\hline Edelberto Santos Dias & a & Elizabeth do Nascimento & - \\
\hline Eden Ribeiro Freire & a & Elizabeth Ferreira Rangel & a \\
\hline Edenilze Teles Romeiro & $\mathrm{c}$ & Elizabeth Gloria Oliveira Barbosa Santos & $\mathrm{a}$ \\
\hline Edesio Jose Tenorio de Melo & a & Elizabeth Igne Ferreira & a \\
\hline Edgar Marcelino de Carvalho Filho & $\mathrm{a}$ & Elizabeth Luz Sanchez Romani & $\mathrm{c}$ \\
\hline Edilaine Marcia Fernandes Camargo & $\mathrm{c}$ & Elizabeth Malagueno de Santana & $\mathrm{c}$ \\
\hline Edilene Alcantara de Castro & $\mathrm{a}$ & Elizabeth Spangler Andrade Moreira & e \\
\hline Edilene Oliveira da Silva & a & Ellen de Souza Marques & - \\
\hline Edmundo Carlos Grisard & a & Ellen Synthia Fernandes de Oliveira & $\mathrm{c}$ \\
\hline Edna Barbosa de Souza & a & Elmo Eduardo de Almeida Amaral & $\mathrm{a}$ \\
\hline Edna Freymuller Haapalainen & a & Eloi de Souza Garcia & $\mathrm{a}$ \\
\hline Edson Luiz Paes Camandaroba & $\mathrm{c}$ & Eloisa Amalia Vieira Ferro & $\mathrm{a}$ \\
\hline Edson Roberto da Silva & a & Eloisa Freitas & $\mathrm{d}$ \\
\hline Edson Rondinelli & $\mathrm{a}$ & Eloy Guillermo Castellon Bermudez & $\mathrm{a}$ \\
\hline Edson Sidiao de Souza Junior & $\mathrm{c}$ & Elsa Evelia Nieves Blanco & - \\
\hline Eduardo Bastianetto & $\mathrm{d}$ & Elton Jose Rosas de Vasconcelos & $\mathrm{e}$ \\
\hline Eduardo Caio Torres dos Santos & a & Elvio Carlos Moreira & $\mathrm{c}$ \\
\hline Eduardo de Almeida Marques da Silva & a & Elvio Machado da Rocha & $\mathrm{d}$ \\
\hline Eduardo Fonseca Pinto & a & Elvira Maria S Chequer Bou Habib & $\mathrm{a}$ \\
\hline Eduardo Henrique Gomes Rodrigues & a & Elza Ferreira Noronha & a \\
\hline Eduardo Jose Lopes Torres & $\mathrm{c}$ & Emanuelle Baldo Gaspar & e \\
\hline Eduardo Lani Volpe da Silveira & a & Emerson Augusto Castilho Martins & e \\
\hline Eduardo Martins Netto & $\mathrm{c}$ & Emilia Akemi Shiraishi Kimura & $\mathrm{a}$ \\
\hline
\end{tabular}




\begin{tabular}{|c|c|c|c|}
\hline Name & Group & Name & Group \\
\hline Eneida Cesar Mastrantonio & $\mathrm{a}$ & Falqueto Aloisio & $\mathrm{a}$ \\
\hline Eneida Willcox Rego & $\mathrm{c}$ & Fanny Beatriz Zamora Veyl & - \\
\hline Enrique Roberto Arganaraz & - & Fanny Tzelepis & - \\
\hline Eric Faudry & $\mathrm{a}$ & Fatima Heritier Corvalan & e \\
\hline Erica Araujo Mendes & $\mathrm{a}$ & Fatima Soares Motta Noronha & $\mathrm{a}$ \\
\hline Erica dos Santos Martins Duarte & a & Felipe de Almeida Dias & a \\
\hline Erica Paes Barreto Xavier Moraes & a & Felipe de Mello Vigoder & $\mathrm{a}$ \\
\hline Erich Loza Telleria & $\mathrm{a}$ & Ferdinan Almeida Melo & $\mathrm{a}$ \\
\hline Erick Vaz Guimaraes & a & Fernanda Barbosa Nogueira & a \\
\hline Erico Vinicius de Souza Carmo & $d$ & Fernanda Caldas Cardoso & $\mathrm{c}$ \\
\hline Erik Daemon de Souza Pinto & $\mathrm{c}$ & Fernanda Cristina Macedo Rondon & $\mathrm{d}$ \\
\hline Erika Hellena Esther Hoffmann & $\mathrm{d}$ & Fernanda Fortes de Araujo & $\mathrm{a}$ \\
\hline Erika Izumi & a & Fernanda Janku Cabral & $\mathrm{c}$ \\
\hline Erika Maria Kopp Xavier da Silveira & e & Fernanda Magalhaes Freire Campos & - \\
\hline Erika Martins Braga & a & Fernanda Maria Santiago & a \\
\hline Erika Michalsky Monteiro & a & Fernanda Nobre Amaral Villani & e \\
\hline Ermelinda Maria Reis Domingues de Carvalho & - & Fernanda Oliveria Novais & - \\
\hline Ernesto Marcus & $\mathrm{c}$ & Fernanda Rosalinski Moraes & $\mathrm{c}$ \\
\hline Erney F Plessmann de Camargo & a & Fernando Alves de Lima Franco & $\mathrm{d}$ \\
\hline Esteban Mauricio Cordero Veas & a & Fernando Antonio Botoni & a \\
\hline Estela Maris Andrade Forell Bevilacqua & $\mathrm{c}$ & Fernando Araujo Monteiro & $\mathrm{a}$ \\
\hline Estevam Guilherme Lux Hoppe & $\mathrm{c}$ & Fernando Augusto de Freitas & $\mathrm{d}$ \\
\hline Etel Rocha Vieira & $\mathrm{c}$ & Fernando Braga Stheling Dias & - \\
\hline Ethan Menahen Shevach & - & Fernando Costa E Silva Filho & $\mathrm{e}$ \\
\hline Eufrosina Setsu Umezawa & a & Fernando de Almeida Borges & $\mathrm{c}$ \\
\hline Eumenia da Costa Cunha Castro & $\mathrm{c}$ & Fernando de Macedo Dossin & $\mathrm{d}$ \\
\hline Eunice Aparecida Bianchi Galati & a & Fernando de Pilla Varotti & $\mathrm{a}$ \\
\hline Eurione Antonio Garcia da Veiga Jardim & $\mathrm{d}$ & Fernando de Queiroz Cunha & $\mathrm{c}$ \\
\hline Eustaquio Claret dos Santos & $\mathrm{e}$ & Fernando Delgado Pretel & $\mathrm{d}$ \\
\hline Evaldo Ferreira Vilela & $\mathrm{c}$ & Fernando Dias de Avila Pires & $\mathrm{a}$ \\
\hline Evaldo Nascimento & $\mathrm{c}$ & Fernando Luiz de Lucca & $\mathrm{c}$ \\
\hline Evander de Jesus Oliveira Batista & $\mathrm{a}$ & Fernando Orefice & $\mathrm{c}$ \\
\hline Eveline Gomes Vasconcelos & a & Fernando Pavao & $\mathrm{a}$ \\
\hline Evelise de Souza Monteiro Fonseca & $\mathrm{c}$ & Fernando Queiroz de Almeida & $\mathrm{c}$ \\
\hline Evelyn Kety Pratt Riccio & a & Filipe Dantas Torres & $\mathrm{a}$ \\
\hline Everton Botelho Sougey & $\mathrm{c}$ & Filomena MP Balestieri & - \\
\hline Fabiana Maria Ruiz Lopes & $\mathrm{c}$ & Firmino Torres de Castro & $\mathrm{a}$ \\
\hline Fabiana Rodrigues dos Santos & a & Flavia Andrade Chaves Borges & $\mathrm{d}$ \\
\hline Fabiana Simao Machado & $\mathrm{a}$ & Flavia Aparecida de Oliveira & $\mathrm{c}$ \\
\hline Fabiane Matos dos Santos & $\mathrm{a}$ & Flavia Aparecida Paina & $\mathrm{c}$ \\
\hline Fabiano Borges Figueiredo & $\mathrm{a}$ & Flavia Coelho Ribeiro & $\mathrm{d}$ \\
\hline Fabiano Sviatopolk Mirsky Pais & e & Flavia da Silva Nader Motta & - \\
\hline Fabio Aguiar Alves & $\mathrm{d}$ & Flavia de Almeida Vieira & $\mathrm{d}$ \\
\hline Fabio Barbour Scott & $\mathrm{c}$ & Flavia Falci Ercole & $\mathrm{c}$ \\
\hline Fabio Husemann Menezes & $\mathrm{c}$ & Flavia Guimaraes Rodrigues & $\mathrm{a}$ \\
\hline Fabio Luiz D Alexandri & - & Flavia Regina Almeida Campos & $\mathrm{a}$ \\
\hline Fabio Marcio Squina & e & Flavio Alves Lara & $\mathrm{a}$ \\
\hline Fabio Mitsuo Lima & $\mathrm{d}$ & Flavio Antonio Pacheco de Araujo & $\mathrm{c}$ \\
\hline Fabio Ribeiro da Silva & $\mathrm{c}$ & Flavio Henrique Beraldo de Paiva & e \\
\hline Fabio Rinaldo Santori & - & Flavya Mendes de Almeida & $\mathrm{c}$ \\
\hline Fabio Trindade Maranhao Costa & a & Flor Ernestina Martinez Espinosa & $\mathrm{a}$ \\
\hline Fabiola Barbieri Holetz & a & Floriano Paes Silva Junior & $\mathrm{c}$ \\
\hline Fabiola Cardillo & a & Francisco Acacio Alves & $\mathrm{a}$ \\
\hline Fabiola do Nascimento Correa & $\mathrm{c}$ & Francisco Carlos Rodrigues de Oliveira & a \\
\hline Fabricia Helena Santello & $\mathrm{d}$ & Francisco de Carvalho Dias Filho & $\mathrm{a}$ \\
\hline Fabricio Cesar Dias & a & Francisco Duque de Mesquita Neto & $\mathrm{c}$ \\
\hline Fabricio Freire de Melo & $\mathrm{c}$ & Francisco Jeronymo Salles Lara & $\mathrm{c}$ \\
\hline Fabricio Klerynton Marchini & a & Francisco Jose Queiroz Monte & $\mathrm{c}$ \\
\hline Fabricio Montalvao Ferreira & e & Francisco Juarez Ramalho Pinto & $\mathrm{a}$ \\
\hline
\end{tabular}




\begin{tabular}{|c|c|c|c|}
\hline Name & Group & Name & Group \\
\hline Francisco Marlon Carneiro Feijo & $\mathrm{c}$ & Guilherme Costa Matsutani & $\mathrm{a}$ \\
\hline Francisco Martins Teixeira & $\mathrm{c}$ & Guilherme Loureiro Werneck & a \\
\hline Franco Claudio Bonetti & $\mathrm{a}$ & Gustavo Adolfo Sierra Romero & $\mathrm{c}$ \\
\hline Francois Noireau & $\mathrm{b}$ & Gustavo Carlos Heringer & - \\
\hline Franklin Barbalho Magalhaes & $\mathrm{a}$ & Gustavo Chemale & $\mathrm{c}$ \\
\hline Franklin Federico Mujica Linarez & - & Gustavo Coutinho Cerqueira & $\mathrm{a}$ \\
\hline Frederico Guilherme Coutinho Abath & $\mathrm{a}$ & Gustavo Ferreira Martins & $\mathrm{c}$ \\
\hline Fredy Roberto Salazar Gutierrez & $\mathrm{b}$ & Gustavo Fontes Paz & a \\
\hline Gabriel Antonio Nogueira Nascentes & a & Gustavo Henrique Goulart Trossini & $\mathrm{a}$ \\
\hline Gabriel Eduardo Melim Ferreira & $\mathrm{a}$ & Gustavo Miranda Rocha & $\mathrm{a}$ \\
\hline Gabriel Grimaldi Filho & a & Habib Frahia Neto & - \\
\hline Gabriel Melo de Oliveira & e & Handel Goncalves Nogueira Oliveira Busatti & - \\
\hline Gary A Splitter & $\mathrm{c}$ & Hatisaburo Masuda & a \\
\hline Geane Peroni Brandao & - & Hector Barrabin & $\mathrm{a}$ \\
\hline Geisa Baptista Barros & a & Hector Francisco Terenzi & $\mathrm{c}$ \\
\hline Geisy Monteiro de Almeida & $\mathrm{d}$ & Heitor Franco de Andrade Junior & $\mathrm{a}$ \\
\hline George Alexandre dos Reis & a & Heitor Miraglia Herrera & $\mathrm{a}$ \\
\hline George Rego Albuquerque & a & Helcileia Dias Santos & a \\
\hline Georgia Correa Atella & a & Helcio Resende Borba & $\mathrm{c}$ \\
\hline Geovania Maria da Silva Braga & $\mathrm{c}$ & Helder Magno Silva Valadares & a \\
\hline Geraldo Gileno de Sa Oliveira & $\mathrm{c}$ & Helen Rodrigues Martins & a \\
\hline Gerhard Wunderlich & a & Helena Bonciani Nader & $\mathrm{c}$ \\
\hline Germano Carneiro da Costa & $\mathrm{d}$ & Helena Heloisa Paixao & $\mathrm{c}$ \\
\hline Gerson Salay & $\mathrm{a}$ & Helena Hilomi Taniguchi & a \\
\hline Gervasio Henrique Bechara & $\mathrm{c}$ & Helena Keiko Toma & $\mathrm{e}$ \\
\hline Giane Amaral de Oliveira & - & Helena Lucia Carneiro Santos & $\mathrm{a}$ \\
\hline Giani Franca Santoro & a & Helena Teru Takahashi & e \\
\hline Gifone Aguiar Rocha & $\mathrm{c}$ & Helene Santos Barbosa & $\mathrm{a}$ \\
\hline Gil Fernando da Costa Mendes De Salles & $\mathrm{c}$ & Helia Maria Cannizzaro & $\mathrm{d}$ \\
\hline Gil Vicente Oliveira da Silva & $\mathrm{d}$ & Helio Langoni & $\mathrm{a}$ \\
\hline Gilberto Garcia Botelho & $\mathrm{c}$ & Helio Santa Rosa Costa Silva & $\mathrm{d}$ \\
\hline Gilberto Mendes de Oliveira Castro & $\mathrm{c}$ & Heloisa Cristina da Silva & $\mathrm{c}$ \\
\hline Gilberto Weissmuller & $\mathrm{c}$ & Heloisa Werneck de Macedo & $\mathrm{c}$ \\
\hline Gilcilene Maria dos Santos & $\mathrm{e}$ & Helton da Costa Santiago & $\mathrm{e}$ \\
\hline Gilda Maria Sales Barbosa & $\mathrm{d}$ & Henio Godeiro Lacerda & $\mathrm{a}$ \\
\hline Gilson Pereira de Oliveira & $\mathrm{c}$ & Henrique Bunselmeyer Ferreira & $\mathrm{c}$ \\
\hline Gilvania Coutinho Silva Feijo & $\mathrm{d}$ & Henrique Couto Teixeira & $\mathrm{c}$ \\
\hline Ginelza Peres Lima dos Santos & a & Henrique de Paula Lemos & $\mathrm{c}$ \\
\hline Giovanna Negromonte Torquato & $\mathrm{d}$ & Heonir De Jesus Pereira da Rocha & $\mathrm{c}$ \\
\hline Girley Francisco Machado de Assis & $\mathrm{a}$ & Heonir Rocha & $\mathrm{c}$ \\
\hline Gisele Fernanda Assine Picchi & $\mathrm{a}$ & Herbert Leonel de Matos Guedes & $\mathrm{a}$ \\
\hline Gisele Maria de Andrade & $\mathrm{c}$ & Herbert Rodrigues Goulart & e \\
\hline Giselle Maria Rachid Viana & a & Hermann Goncalves Schatzmayr & $\mathrm{c}$ \\
\hline Gislaine Aparecida Martins & $\mathrm{a}$ & Hermione Elly Melara de Campos Bicudo & $\mathrm{c}$ \\
\hline Giulliano Aires Anderlini & $\mathrm{d}$ & Hernan Chaimovich & $\mathrm{c}$ \\
\hline Glaucia Manzan Queiroz de Andrade & - & Hernando Del Portillo & $\mathrm{a}$ \\
\hline Glaucia Noemy Rodrigues Vespa & - & Hertha Meyer & a \\
\hline Glaucie Jussilane Alves & $\mathrm{c}$ & Herton Helder Rocha Pires & $\mathrm{c}$ \\
\hline Glaucius Oliva & a & Hideko Yamanaka & $\mathrm{c}$ \\
\hline Glenda Meira Cardoso & a & Hilda Fatima de Jesus Pena & a \\
\hline Glenio Cavalcanti de Barros & $\mathrm{c}$ & Hilton Ribeiro da Rocha & - \\
\hline Gloria Cadavid Restrepo & a & Hiro Goto & a \\
\hline Gloria Regina Franco & $\mathrm{a}$ & Hooman Momen & $\mathrm{a}$ \\
\hline Gordon Lewis Edwin Koch & - & Hugo Caire de Castro Faria Neto & $\mathrm{a}$ \\
\hline Graziela Maria Zanini & $\mathrm{a}$ & Humberto de Araujo Rangel & $\mathrm{a}$ \\
\hline Greice Krautz de Mello & - & Idessania Nazareth da Costa & $\mathrm{a}$ \\
\hline Guacyara Tenorio Cavalcante & $\mathrm{c}$ & Ieda Maria Martinez Paino & $\mathrm{c}$ \\
\hline Guido Fontgalland Coelho Linhares & a & Igor de Almeida Rodrigues & $\mathrm{a}$ \\
\hline Guilherme Correa de Oliveira & $\mathrm{d}$ & Ilea Brandao Rodrigues & a \\
\hline
\end{tabular}




\begin{tabular}{|c|c|c|c|}
\hline Name & Group & Name & Group \\
\hline Ingrid Solange Evans Osses & $\mathrm{a}$ & Jean Dausset & - \\
\hline Ionice Felipe & $\mathrm{c}$ & Jean Yveslaronze & - \\
\hline Iramaya Rodrigues Caldas & $\mathrm{c}$ & Jeanne Blanco de Molfetta & $\mathrm{c}$ \\
\hline Irene da Silva Soares & $\mathrm{a}$ & Jeffrey Jon Shaw & $\mathrm{a}$ \\
\hline Isaac Roitman & $\mathrm{a}$ & Jenner Karlisson Pimenta dos Reis & $\mathrm{c}$ \\
\hline Isabel Albuquerque Porto Carreiro & $d$ & Jeronimo Conceicao Ruiz & a \\
\hline Isabel Barroso Augusto da Silva & - & Jerusa Marilda Arantes & $\mathrm{e}$ \\
\hline Isabel Cristina Fabregas Bonna & a & Jesus Santiago Moure & $\mathrm{c}$ \\
\hline Isabel Martinez & a & Joana Ferreira do Amaral & $\mathrm{e}$ \\
\hline Isabela Penna Ceravolo & a & Joanna Darc Aparecida Herzog Soares & $\mathrm{a}$ \\
\hline Isabele Barbieri dos Santos & $\mathrm{d}$ & Joao Aguiar Nogueira Batista & $\mathrm{e}$ \\
\hline Isabella Dib Ferreira Gremiao & $\mathrm{c}$ & Joao Alves Araujo Filho & $\mathrm{c}$ \\
\hline Isaias Raw & $\mathrm{c}$ & Joao Aristeu da Rosa & $\mathrm{c}$ \\
\hline Ises de Almeida Abrahamsohn & $\mathrm{a}$ & Joao Atilio Jorge & $\mathrm{c}$ \\
\hline Isis Fernandes Magalhaes Santos & $\mathrm{a}$ & Joao Carlos Araujo Carreira & $\mathrm{a}$ \\
\hline Italmar Teodorico Navarro & a & Joao Carlos Gonzales & $\mathrm{c}$ \\
\hline Italo Rodrigues De Araujo Sherlock & $\mathrm{a}$ & Joao Carlos Palazzo de Mello & $\mathrm{c}$ \\
\hline Ivan da Mota E Albuquerque & $\mathrm{c}$ & Joao Carlos Pinto Dias & $\mathrm{a}$ \\
\hline Ivan M Roitt & - & Joao Elias Vidueira Ferreira & $\mathrm{d}$ \\
\hline Ivan Teobaldo Neira Cortes & - & Joao Garcia Leme & $\mathrm{c}$ \\
\hline Ivan Vieira Sonoda & $\mathrm{d}$ & Joao Luis Garcia & $\mathrm{a}$ \\
\hline Ivana Nunes Gomes & a & Joao Luiz Ferreira & $\mathrm{c}$ \\
\hline Ivete Conchon Costa & $\mathrm{a}$ & Joao Luiz Horacio Faccini & $\mathrm{c}$ \\
\hline Ivna Alana Freitas Brasileiro da Silveira & $\mathrm{c}$ & Joao Luiz Mendes Wanderley & $\mathrm{a}$ \\
\hline Ivo Santana Caldas & $\mathrm{a}$ & Joao Morelli Junior & $\mathrm{c}$ \\
\hline Ivonise Follador de Oliveira & - & Joao Palermo Neto & $\mathrm{c}$ \\
\hline Izabela Marques Doutrado Bastos & - & Joao Roberto da Mata & $\mathrm{a}$ \\
\hline Izabella Cabral Hassum & $\mathrm{c}$ & Joao Santana da Silva & $\mathrm{a}$ \\
\hline Izaltina Silva Jardim & a & Joao Soares Moreira & $\mathrm{e}$ \\
\hline Izilda Curado & $\mathrm{a}$ & Joao Tadeu Ribeiro Paes & $\mathrm{c}$ \\
\hline Jacenir Reis dos Santos Mallet & a & Joaquin Hernan Patarroyo Salcedo & $\mathrm{a}$ \\
\hline Jacira Maria Andrade de Sousa & $\mathrm{e}$ & Joel Alves Lamounier & $\mathrm{c}$ \\
\hline Jackson Machado Pinto & $\mathrm{c}$ & John F Peberdy & - \\
\hline Jacqueline Isaura Alvarez Leite & $\mathrm{c}$ & John Leslie Turk & $\mathrm{b}$ \\
\hline Jacques Robert Nicoli & $\mathrm{c}$ & Jolande Disch & - \\
\hline Jacy Gameiro & $\mathrm{c}$ & Jonh Furlong & - \\
\hline Jaime Martins de Santana & $\mathrm{a}$ & Jorge Alberto Lopez Rodriguez & $\mathrm{e}$ \\
\hline Jair Pereira da Cunha Junior & a & Jorge Almeida Guimaraes & $\mathrm{c}$ \\
\hline Jair Rodine Engracia Filho & - & Jorge Andrade Pinto & $\mathrm{c}$ \\
\hline Jairo Dias Barreira & a & Jorge Cardoso Messeder & $\mathrm{e}$ \\
\hline Jairo Pinheiro da Silva & $\mathrm{c}$ & Jorge Clarencio Souza Andrade & $\mathrm{a}$ \\
\hline Jaline Coutinho Silverio & $\mathrm{d}$ & Jorge Elias Kalil Filho & $\mathrm{a}$ \\
\hline James Harvey Maguire & $\mathrm{b}$ & Jorge Luiz Pesquero & $\mathrm{c}$ \\
\hline Jamile Prado dos Santos & e & Jorge Marcelo de Freitas & $\mathrm{d}$ \\
\hline Jan Carlo Morais Oliveira B Delorenzi & a & Jose Adail Fonseca de Castro & $\mathrm{a}$ \\
\hline Janaina Claudia da Silva Saldanha & $\mathrm{d}$ & Jose Ailton da Silva & a \\
\hline Janaina Fernanda Goncalves Neto & e & Jose Alberto Sampaio Nunes de Mello & - \\
\hline Janaina Sousa Campos & $\mathrm{d}$ & Jose Almir Moraes da Rocha & $\mathrm{a}$ \\
\hline Janayna Hammes de Almeida & $\mathrm{d}$ & Jose Andries Morgado Diaz & - \\
\hline Jane Lima dos Santos & a & Jose Angelo Lauletta Lindoso & $\mathrm{a}$ \\
\hline Janete Chung & e & Jose Bento Pereira Lima & $\mathrm{a}$ \\
\hline Janete Soares Coelho dos Santos & a & Jose Borges Pereira & e \\
\hline Janethe Deolina de Oliveira Pena & $\mathrm{c}$ & Jose Carlos Miranda & $\mathrm{a}$ \\
\hline Janeusa Trindade de Souto & $\mathrm{c}$ & Jose Carlos Pereira de Souza & $\mathrm{c}$ \\
\hline Jaqueline Brandao Guerreiro Marotti & $\mathrm{c}$ & Jose Carlos Serufo & $\mathrm{c}$ \\
\hline Jayme Angluster & a & Jose Ciriaco Pinheiro & $\mathrm{c}$ \\
\hline Jayme Augusto de Souza Neto & $\mathrm{a}$ & Jose Clovis do Prado Junior & $\mathrm{a}$ \\
\hline Jayme Neves & a & Jose da Rocha Carvalheiro & a \\
\hline Jean Antoine Rioux & $\mathrm{b}$ & Jose Daniel Figueroa Villar & a \\
\hline
\end{tabular}




\begin{tabular}{|c|c|c|c|}
\hline Name & Group & Name & Group \\
\hline Jose Daniel Lopes & $\mathrm{c}$ & Juliana Martha Sa & - \\
\hline Jose de Angelis Cortes & - & Juliana Natal Amazonas & $\mathrm{d}$ \\
\hline Jose de Moura Goncalves & $\mathrm{c}$ & Juliana Perrone Bezerra de Menezes & $\mathrm{a}$ \\
\hline Jose de Ribamar de Castro Carvalho & $\mathrm{d}$ & Juliana Ramos Pimenta & $\mathrm{d}$ \\
\hline Jose Divino Lima & $\mathrm{c}$ & Juliana Saraiva & $\mathrm{d}$ \\
\hline Jose Eduardo Serrao & $\mathrm{c}$ & Juliano Simoes de Toledo & $\mathrm{a}$ \\
\hline Jose Eduardo Tolezano & $\mathrm{a}$ & Juliany Cola Fernandes Rodrigues & $\mathrm{a}$ \\
\hline Jose Eloy dos Santos Junior & $\mathrm{e}$ & Julio Cesar Carranza Martinez & - \\
\hline Jose Eymard Homem Pitella & - & Julio Cesar Mine & $\mathrm{c}$ \\
\hline Jose Franco da Silveira Filho & $\mathrm{a}$ & Julio Cesar Rocha Costa & $\mathrm{c}$ \\
\hline Jose Henrique Maia Campos de Oliveira & a & Julio Cesar Soares Aliberti & $\mathrm{e}$ \\
\hline Jose Jurberg & $\mathrm{a}$ & Julio Flavio Meirelles Marchini & $\mathrm{e}$ \\
\hline Jose Leal Prado & $\mathrm{c}$ & Julio Pudles & a \\
\hline Jose Lino Neto & $\mathrm{c}$ & Julio Scharfstein & $\mathrm{a}$ \\
\hline Jose Luciano Nepomuceno da Silva & a & Junia de Sousa Franco & $\mathrm{a}$ \\
\hline Jose Luiz de Barros Araujo & $\mathrm{c}$ & Karen Angelica Cavassani de Souza & $\mathrm{a}$ \\
\hline Jose Luiz Martins do Nascimento & $\mathrm{c}$ & Karen dos Santos Charret & $\mathrm{a}$ \\
\hline Jose Manuel Macario Rebelo & a & Karen Luisa Haag & $\mathrm{c}$ \\
\hline Jose Marcelo Ramalho Ortigao & a & Karin Kirchgatter & $\mathrm{a}$ \\
\hline Jose Marcio Sbruzzi Cardoso & $\mathrm{d}$ & Karina Nogueira Zeviani & - \\
\hline Jose Maria Alvarez Mosig & $\mathrm{a}$ & Karina Penedo Carvalho & a \\
\hline Jose Maria de Souza & a & Karine Rezende de Oliveira & $\mathrm{a}$ \\
\hline Jose Maria Segovia de Arana & - & Karis Maria de Pinho Rodrigues & $\mathrm{e}$ \\
\hline Jose Maria Soares Barata & a & Karla Consort Ribeiro & $\mathrm{c}$ \\
\hline Jose Mario de Freitas Balanco & $\mathrm{e}$ & Katia Cirlene Alves Botelho & $\mathrm{c}$ \\
\hline Jose Mauro Peralta & a & Katia da Silva Calabrese & a \\
\hline Jose Miguel Ortega & $\mathrm{c}$ & Katia Denise Saraiva Bresciani & e \\
\hline Jose Orivaldo Mengele Junior & $\mathrm{c}$ & Katia Roberta Fernandes & $\mathrm{c}$ \\
\hline Jose Osvaldo Costa & - & Katia Solange Cardoso Rodrigues dos Santos & $\mathrm{e}$ \\
\hline Jose Osvaldo Previato & a & Katyane de Sousa Almeida & $\mathrm{e}$ \\
\hline Jose Raimundo Correa & $\mathrm{e}$ & Kelly Aparecida Geraldo Yoneyama & $\mathrm{a}$ \\
\hline Jose Ribamar Mesquita Teixeira & a & Kelly Salomao & a \\
\hline Jose Roberto Meyer Fernandes & a & Kelsen Dantas Eulalio & $\mathrm{c}$ \\
\hline Jose Roberto Mineo & $\mathrm{a}$ & Kennedy Kiriira Gachoka & - \\
\hline Jose Rodrigues Coura & $\mathrm{a}$ & Kenneth John Gollob & $\mathrm{b}$ \\
\hline Jose Rodrigues da Silva & a & Kezia Katiani Gorza Scopel & a \\
\hline Jose Ronnie Carvalho de Vasconcelos & $\mathrm{a}$ & Kildare Rocha de Miranda & $\mathrm{a}$ \\
\hline Jose Wilton Pinheiro Junior & $\mathrm{e}$ & Killarney Ataide Soares & $\mathrm{d}$ \\
\hline Joseli de Oliveira Ferreira & a & Klaus Scherrer & $\mathrm{c}$ \\
\hline Joseli Lannes Vieira & $\mathrm{a}$ & Klaus Wrogemann & - \\
\hline Josiana Gomes de Andrade & $\mathrm{c}$ & Laerte Bento Viola & e \\
\hline Josiane Cardoso & e & Laerte Grisi & $\mathrm{a}$ \\
\hline Joyce Eliza de Oliveira Souza & - & Lain Carlos Pontes de Carvalho & a \\
\hline Juan Miguel Villalobos Salcedo & $\mathrm{a}$ & Lais de Carvalho & $\mathrm{a}$ \\
\hline Jucara Fahrat de Carvalho Parra & - & Lanny Cristina Burlandy Soares & $\mathrm{c}$ \\
\hline Judith Felcman & $\mathrm{c}$ & Larry Simpson & $\mathrm{b}$ \\
\hline Judith Kardos Kloetzel & a & Laura C Barbosa de Oliveira & - \\
\hline Judith Tibisay Molina David & $\mathrm{d}$ & Laura Nogueira da Cruz & $\mathrm{a}$ \\
\hline Julia Maria Costa Cruz & $\mathrm{c}$ & Lea Camillocoura & - \\
\hline Julia Pinheiro Chagas da Cunha & a & Lea Cysne Finkelstein & a \\
\hline Juliana Cristina Schmidt & $\mathrm{d}$ & Lea Ferreira Camillo Coura & $\mathrm{a}$ \\
\hline Juliana da Matta Furniel Dutra & $\mathrm{e}$ & Leandro Lemgruber Soares & $\mathrm{a}$ \\
\hline Juliana de Assis Silva Gomes Estanislau & $\mathrm{a}$ & Leda Quercia Vieira & $\mathrm{a}$ \\
\hline Juliana de Meis & a & Leidi Maria Herrera Cabrera & - \\
\hline Juliana Dias Costa Roque & $\mathrm{a}$ & Leila de Mendonca Lima & $\mathrm{a}$ \\
\hline Juliana Giantomassi Machado & $\mathrm{e}$ & Leila Ines Aguiar Raposo da Camara Coelho & $\mathrm{a}$ \\
\hline Juliana Gonzaga de Oliveira & a & Leila Maria Silva Lopes & $\mathrm{e}$ \\
\hline Juliana Hipolito Pessoti & - & Leila Saddi Ortega & $\mathrm{d}$ \\
\hline Juliana Lopes Rangel Fietto & a & Lenita Ramires dos Santos & $\mathrm{e}$ \\
\hline
\end{tabular}




\begin{tabular}{|c|c|c|c|}
\hline Name & Group & Name & Group \\
\hline Leonardo Augusto de Almeida & $\mathrm{c}$ & Luciana Mori Viero & $\mathrm{c}$ \\
\hline Leonardo de Souza Rocha & $\mathrm{a}$ & Luciana Regina Meireles & $\mathrm{a}$ \\
\hline Leonardo Freire de Lima & a & Luciana Ribeiro Garzoni & a \\
\hline Leonardo Jose de Moura Carvalho & $\mathrm{a}$ & Luciana Rodrigues de Almeida & a \\
\hline Leonardo Jose Richtzenhain & $\mathrm{c}$ & Luciana Teixeira Zimmermann & $\mathrm{a}$ \\
\hline Leonardo Nimrichter & $\mathrm{c}$ & Luciana Urbano dos Santos & a \\
\hline Leonardo Pereira Quintella & $\mathrm{c}$ & Luciana Vignolio Alves & $\mathrm{d}$ \\
\hline Leoni Villano Bonami & - & Luciana Weidlich & $\mathrm{c}$ \\
\hline Leonidas de Mello Deane & $\mathrm{a}$ & Luciane Moreno Storti de Melo & a \\
\hline Leonor Bezerra Guerra & $\mathrm{c}$ & Luciane Rodrigues Portugal & d \\
\hline Leonor Garcia Rincon & $\mathrm{c}$ & Luciano Andrade Moreira & a \\
\hline Leonor Laura Pinto Leon & $\mathrm{a}$ & Luciano Aparecido Panagio & e \\
\hline Leony Cristina Caetano & $\mathrm{d}$ & Luciano Melo de Souza & $\mathrm{d}$ \\
\hline Leopoldo de Meis & $\mathrm{c}$ & Luciene Chaves Fernandes & $\mathrm{c}$ \\
\hline Leucio Camara Alves & $\mathrm{a}$ & Lucila Grossi Goncalves Pacifico & $\mathrm{c}$ \\
\hline Lia Carolina Almeida Soares de Medeiros & $\mathrm{a}$ & Lucile Maria Floeter Winter & a \\
\hline Liana Cardoso Verinaud & $\mathrm{a}$ & Lucilene Amorim Silva & a \\
\hline Liane Casagrande & $\mathrm{d}$ & Lucilene Simoes Mattos & $\mathrm{d}$ \\
\hline Lidia Maria Pepe de Moraes & $\mathrm{c}$ & Lucimar Goncalves Milagres & e \\
\hline Lidiane Aparecida Pereira de Sousa & e & Lucimeire Antonelli da Silveira & a \\
\hline Ligia Miranda Ferreira Borges & $\mathrm{c}$ & Lucio Andre Viana Dias & a \\
\hline Lileia Goncalves Diotaiuti & $\mathrm{a}$ & Lucio Ayres Caldas & a \\
\hline Liliam de Oliveira Faria Macaneiro & $\mathrm{a}$ & Lucio Holanda Gondim de Freitas Junior & a \\
\hline Lilian Lacerda Bueno & a & Lucio Roberto Cancado Castellano & a \\
\hline Lilian Maria Castilho & e & Lucy Megumi Yamauchi Lioni & a \\
\hline Lilian Maria Reis Afonso & - & Ludmila Fiorenzano Baethgen & $\mathrm{c}$ \\
\hline Liliana Torcoroma Garcia Sanchez & $\mathrm{d}$ & Ludmila Rodrigues Pinto Ferreira & a \\
\hline Liliane Coelho da Rocha Nery & $\mathrm{a}$ & Luigi Bogliolo & - \\
\hline Liliane Martins dos Santos & $\mathrm{e}$ & Luis Andre Pontes & $\mathrm{c}$ \\
\hline Linda Khalili Boukai & - & Luis Carlos Crocco Afonso & $\mathrm{a}$ \\
\hline Lindamar Maria de Souza Minelli & - & Luis Eduardo Ramirez Giraldo & a \\
\hline Lindsay Ann Pirrit & $\mathrm{d}$ & Luis Fernando Santana & a \\
\hline Lis Ribeiro do Valle Antonelli & $\mathrm{e}$ & Luis Natividad Nunez Apaza & - \\
\hline Lizandra Guidi Magalhaes & $\mathrm{a}$ & Luisa Damazio Rona Pitaluga & a \\
\hline Loic Monjour & $\mathrm{b}$ & Luiz Alberto Santana & $\mathrm{a}$ \\
\hline Loislene Oliveira Brito & $\mathrm{e}$ & Luiz Antonio Franco da Silva & $\mathrm{c}$ \\
\hline Loraine Campanati Araujo de Andrade & e & Luiz Antonio Rodrigues de Freitas & $\mathrm{a}$ \\
\hline Lourdes Maria Garcez dos Santos Silveira & $\mathrm{a}$ & Luiz Augusto Batista Brito & $\mathrm{c}$ \\
\hline Luci Ana Fernandes Martins & - & Luiz Augusto Magalhaes & $\mathrm{c}$ \\
\hline Lucia Eiko Oishi Yai & - & Luiz Augusto Pinto & $d$ \\
\hline Lucia Helena Odwyer de Oliveira & $\mathrm{a}$ & Luiz Caetano da Silva & $\mathrm{c}$ \\
\hline Lucia Helena Pinto da Silva & $\mathrm{a}$ & Luiz Candido de Souza Dias & $\mathrm{c}$ \\
\hline Lucia Maria da Cunha Galvao & $\mathrm{a}$ & Luiz Carlos Alves & a \\
\hline Lucia Mendonca Previato & $\mathrm{a}$ & Luiz Carlos Duarte Formiga & - \\
\hline Lucia Padilha Cury Thomaz de Aquino & $\mathrm{d}$ & Luiz Carlos Junqueira & $\mathrm{c}$ \\
\hline Luciamare Perinetti Alves Martins & $\mathrm{a}$ & Luiz Claudio Miletti & $\mathrm{a}$ \\
\hline Luciana da Conceicao Pinto & $\mathrm{a}$ & Luiz da Silva Vieira & $\mathrm{c}$ \\
\hline Luciana da Silva Mukai & $\mathrm{c}$ & Luiz de Macedo Farias & $\mathrm{c}$ \\
\hline Luciana de Almeida Silva & a & Luiz Dione Barbosa de Melo & a \\
\hline Luciana de Fatima Sianto Martins & - & Luiz Fabiano Borges Oliveira & a \\
\hline Luciana de Gouvea Viana & e & Luiz Fernando Rocha Ferreira da Silva & a \\
\hline Luciana de Oliveira Andrade & a & Luiz Hildebrando Pereira da Silva & a \\
\hline Luciana Furlaneto & $\mathrm{c}$ & Luiz Marcelo Aranha Camargo & $\mathrm{a}$ \\
\hline Luciana Girotto Gentil & e & Luiz Ricardo Orsini Tosi & a \\
\hline Luciana Lima & $\mathrm{a}$ & Luiz Roberto Sardinha & $\mathrm{a}$ \\
\hline Luciana Lopes de Almeida Ribeiro Garzoni & a & Luiz Rodolpho Raja Gabaglia Travassos & a \\
\hline Luciana Loureiro Penha Pacheco & - & Luiz Vicente Rizzo & a \\
\hline Luciana Madeira da Silva & e & Lukas C Kuhn & - \\
\hline Luciana Maria Silva & $\mathrm{c}$ & Luzia Helena Carvalho & a \\
\hline
\end{tabular}




\begin{tabular}{|c|c|c|c|}
\hline Name & Group & Name & Group \\
\hline Luzineide Wanderley Tinoco & a & Marcio Botelho de Castro & $\mathrm{c}$ \\
\hline Lysangela Ronalte Alves & a & Marcio Loureno Rodrigues & - \\
\hline Magdalena Nascimento Renno & a & Marcio Neves Boia & $\mathrm{c}$ \\
\hline Maira Cegatti Bosetto & a & Marcio Silva & a \\
\hline Manoel Adriao Gomes Filho & $\mathrm{c}$ & Marcio Sobreira S Araujo & - \\
\hline Manoel Barral Netto & a & Marcio Vinicius Lins de Barros & $\mathrm{c}$ \\
\hline Manoel Otavio da Costa Rocha & a & Marco Antonio de Oliveira & e \\
\hline Manoel Paes de Oliveira Neto & a & Marco Antonio Granja Barbosa & $\mathrm{c}$ \\
\hline Manzelio Cavazzana Junior & e & Marco Aurelio Krieger & $\mathrm{a}$ \\
\hline Mara Silvia Carvalhaes & a & Marco Tulio Alves da Silva & a \\
\hline Marcel Ivan Ramirez Araya & a & Marco Tulio Antonio Garciazapata & a \\
\hline Marcel Marin Villa & - & Marcos Andre Vannier dos Santos & $\mathrm{a}$ \\
\hline Marcel Teixeira & a & Marcos Augusto Grigolin Grisotto & a \\
\hline Marcela Barbosa de Figueiredo & $\mathrm{a}$ & Marcos Barbosa de Souza & $\mathrm{a}$ \\
\hline Marcela de Freitas Lopes & a & Marcos Celio de Almeida & $\mathrm{e}$ \\
\hline Marcela Goncalves Drummond & $\mathrm{c}$ & Marcos de Assis Moura & e \\
\hline Marcela Lencine Ferraz & $\mathrm{d}$ & Marcos Gonzaga dos Santos & a \\
\hline Marcela Orsini Andrade & $\mathrm{d}$ & Marcos Henrique Ferreira Sorgine & $\mathrm{a}$ \\
\hline Marcello Andre Barcinski & $\mathrm{a}$ & Marcos Horacio Pereira & $\mathrm{a}$ \\
\hline Marcello Xavier Sampaio & $\mathrm{a}$ & Marcos Jose Marques & $\mathrm{a}$ \\
\hline Marcelo Beltrao Molento & $\mathrm{c}$ & Marcos Leoni Gazarini Dutra & $\mathrm{e}$ \\
\hline Marcelo da Silva Genestra & a & Marcos Pinheiro Franque & a \\
\hline Marcelo Einicker Lamas & a & Marcos Roberto Bonuti & $\mathrm{c}$ \\
\hline Marcelo Luiz Carvalho Goncalves & e & Marcos Rommel Cabanillas Silva & a \\
\hline Marcelo Ribeiro da S Briones & a & Marcos Takashi Obara & $\mathrm{a}$ \\
\hline Marcelo Rodrigues Pinto & $\mathrm{e}$ & Marcos Vinicius da Silva & $\mathrm{a}$ \\
\hline Marcelo Salabert Gonzalez & a & Marcus Fernandes de Oliveira & $\mathrm{a}$ \\
\hline Marcelo Santos Castilho & e & Marcus Vinicius Gouvea & $\mathrm{d}$ \\
\hline Marcelo Tavares Viana & $\mathrm{c}$ & Margaret Haiganouch Magdesian & e \\
\hline Marcelo Urbano Ferreira & $\mathrm{a}$ & Margareth Maria Lessa Goncalves & $\mathrm{c}$ \\
\hline Marcelo Vidigal Caliari & a & Margarida Maria de Lima Pompeu & $\mathrm{c}$ \\
\hline Marcia Alcantara Santos & - & Margo Gomes de Oliveira Karnikowski & - \\
\hline Marcia Almeida de Melo & $\mathrm{a}$ & Margoth Mitchela Moreno Vigo & $\mathrm{d}$ \\
\hline Marcia Antoniazi Michelin & e & Margoth Ramos Garnica & - \\
\hline Marcia Aparecida Duranti & $\mathrm{d}$ & Maria Amelia Villela Oliva & e \\
\hline Marcia Aparecida Speranca & a & Maria Angelica Vieira da Costa Pereira & $\mathrm{c}$ \\
\hline Marcia Attias & $\mathrm{a}$ & Maria Aparecida da Gloria Faustino & $\mathrm{a}$ \\
\hline Marcia Benedita de Oliveira Silva & a & Maria Aparecida da Silva & - \\
\hline Marcia Chame & e & Maria Aparecida Gomes & $\mathrm{a}$ \\
\hline Marcia Cristina Alves Teixeira & $\mathrm{d}$ & Maria Arlene Fausto & $\mathrm{c}$ \\
\hline Marcia Cristina Aquino Teixeira & $\mathrm{a}$ & Maria Auxiliadora de Sousa & $\mathrm{a}$ \\
\hline Marcia Cristina Fornazim & $\mathrm{c}$ & Maria Auxiliadora Pantoja Ferreira & e \\
\hline Marcia Cristina Paes & a & Maria Auxiliadora Roque de Carvalho & - \\
\hline Marcia de Berredo Pinho & e & Maria Beatriz Araujo Silva & $\mathrm{a}$ \\
\hline Marcia de Figueiredo Pereira & $\mathrm{c}$ & Maria Belem Cassera & $\mathrm{b}$ \\
\hline Marcia de Melo Barbosa & $\mathrm{c}$ & Maria Carla Bastos Abdo & - \\
\hline Marcia dos Santos Lazera & $\mathrm{c}$ & Maria Carolina Quartim Barbosa Elias & a \\
\hline Marcia Ferraz Nogueira & - & Maria Cecilia Di Ciero Fernandes & $\mathrm{a}$ \\
\hline Marcia Helena Rodrigues Velloso & $\mathrm{c}$ & Maria Clara Leal Nascimento Silva & $\mathrm{d}$ \\
\hline Marcia Kiyoe Shimada & $\mathrm{a}$ & Maria Clorinda Soares Fioravanti & e \\
\hline Marcia Maria de Negreiros Pinto Rocha & $\mathrm{c}$ & Maria Cristina Casaqui Carollo & $\mathrm{d}$ \\
\hline Marcia Mayumi Ishikawa & $\mathrm{c}$ & Maria Cristina Fortes Santos de Bustamante & $\mathrm{d}$ \\
\hline Marcia Narciso Borges & - & Maria Cristina Machado Motta & $\mathrm{a}$ \\
\hline Marcia Pereira de Oliveira & a & Maria Cristina Nonato & a \\
\hline Marcia Ramos E Silva & $\mathrm{c}$ & Maria Cristina Roque Antunes Barreira & $\mathrm{c}$ \\
\hline Marcia Regina Machado dos Santos & e & Maria da Gloria Bonecini de Almeida & a \\
\hline Marcia Regina Piuvezam & e & Maria da Gloria Bonfim & - \\
\hline Marcia Rosa de Oliveira & a & Maria da Gloria Fernandes Pegado & a \\
\hline Marcia Tosta Xavier & e & Maria da Gloria Gomes Cristino & $\mathrm{c}$ \\
\hline
\end{tabular}




\begin{tabular}{|c|c|c|c|}
\hline Name & Group & Name & Group \\
\hline Maria da Gloria Martins Teixeira & $\mathrm{d}$ & Maria Zilma Andrade Rodrigues & $\mathrm{d}$ \\
\hline Maria da Gloria Quintao E Silva & e & Marialice da Fonseca Ferreira da Silva & e \\
\hline Maria Dalila Forlano Riera & $\mathrm{c}$ & Mariana Caldas Waghabi & a \\
\hline Maria das Gracas Costa Alecrim & $\mathrm{a}$ & Mariana Machado Hecht & $\mathrm{a}$ \\
\hline Maria das Gracas Miranda Danelli & e & Mariane Bandeira de Melo Braga & - \\
\hline Maria das Gracas Prianti & a & Mariangela Carneiro & a \\
\hline Maria de Fatima Costa Alves & e & Mariano Gustavo Zalis & a \\
\hline Maria de Fatima Ferreira da Cruz & a & Mariko Yokoo & a \\
\hline Maria de Fatima Freire de Melo Ximenes & a & Marilene Marcurzzo do Canto Cavalheiro & - \\
\hline Maria de Fatima Madeira & a & Marilene Maria de Lima & a \\
\hline Maria de Fatima Martins Horta & a & Marilene Suzan Marques Michalick & a \\
\hline Maria de Lourdes Petrillo Peixoto & a & Marina de Moraes Mourao & $\mathrm{c}$ \\
\hline Maria de Lourdes Ribeiro de Carvalho & $\mathrm{d}$ & Marina Vianna Braga & $\mathrm{e}$ \\
\hline Maria de Nazare Correia Soeiro & a & Marinete Marins Povoa & a \\
\hline Maria de Nazare Tavares da Silva & a & Marinez Almeida de Faria & $\mathrm{d}$ \\
\hline Maria de Nazareth Silveira Leal de Meirelles & a & Mario Antonio Spano & $\mathrm{e}$ \\
\hline Maria do Amparo Salmito Cavalcanti & - & Mario Endsfeldez Camargo & $\mathrm{a}$ \\
\hline Maria do Carmo Pereira Nunes & $\mathrm{c}$ & Mario Steindel & $\mathrm{a}$ \\
\hline Maria do Socorro Costa Oliveira Braga & $\mathrm{e}$ & Marise Pinheiro Nunes & $\mathrm{a}$ \\
\hline Maria do Socorro Rosa Rodrigues de Carvalho & a & Maristela Gomes da Cunha & a \\
\hline Maria Eduarda Monteiro Silva & $\mathrm{d}$ & Maristela Martins de Camargo & e \\
\hline Maria Emilia Bodini Santiago & $\mathrm{e}$ & Mariza Fordelone Rosa Cruz & - \\
\hline Maria Esther Carvalho & $\mathrm{a}$ & Mariza Martins Avelino & a \\
\hline Maria Esther Macedo & $\mathrm{d}$ & Marlei Joseli Augusto & - \\
\hline Maria Fernanda Laranjeira da Silva & $\mathrm{a}$ & Marlene Antonia dos Reis & $\mathrm{c}$ \\
\hline Maria Fernanda Reis Gavazzoni Dias & $\mathrm{c}$ & Marlene Benchimol & a \\
\hline Maria Helena Franco Morais & $\mathrm{d}$ & Marlene Cabrine dos Santos & $\mathrm{a}$ \\
\hline Maria Heloisa de Souza Lima Blotta & $\mathrm{c}$ & Marlene Isabel Vargas Viloria & $\mathrm{a}$ \\
\hline Maria Ilma Andrade Santos de Araujo & - & Marlene Tiduko Ueta & $\mathrm{c}$ \\
\hline Maria Imaculada Muniz Barboza Junqueira & a & Marlisson Augusto Costa Feitosa & $\mathrm{a}$ \\
\hline Maria Inacia Estevao Costa & $\mathrm{c}$ & Marselha Marques Barral & $\mathrm{c}$ \\
\hline Maria Inez Santos Silva & $\mathrm{e}$ & Marsen Garcia Pinto Coelho & $\mathrm{c}$ \\
\hline Maria Isabel Botelho Vieira & $\mathrm{d}$ & Marta Chagas Monteiro & $\mathrm{a}$ \\
\hline Maria Isabel Lynch Gaete & a & Marta Citelli dos Reis & e \\
\hline Maria Izabel de Pinho Pesso Amorim & - & Marta de Lana & $\mathrm{a}$ \\
\hline Maria Jania Teixeira & a & Marta Maria Geraldes Teixeira & a \\
\hline Maria Jose Conceicao & $\mathrm{c}$ & Marta Tavares Dagosto & $\mathrm{a}$ \\
\hline Maria Julia Manso Alves & a & Marta Teixeira Gomes & $\mathrm{a}$ \\
\hline Maria Julia Salim Pereira & $\mathrm{c}$ & Martha Cecilia Suarez Mutis & $\mathrm{a}$ \\
\hline Maria Leticia Costa Reis & a & Martin Cancela Sehabiague & $\mathrm{c}$ \\
\hline Maria Linda Flora de Novaes Benetton & $\mathrm{c}$ & Martin Nemer & - \\
\hline Maria Lucia Belem Pinheiro & $\mathrm{c}$ & Massayoshi Yoshida & $\mathrm{c}$ \\
\hline Maria Lucia Rosa Rossetti & $\mathrm{c}$ & Matheus Andrade Rajao & $\mathrm{e}$ \\
\hline Maria Lucia Vieira Moreno & a & Mauricio Costa Fonseca Vieira & - \\
\hline Maria Luiza Brito de Sousa Atta & a & Mauricio da Rocha e Silva & $\mathrm{c}$ \\
\hline Maria Luiza Cruz & $\mathrm{e}$ & Mauricio Martins Rodrigues & $\mathrm{a}$ \\
\hline Maria Madalena Pessoa Guerra & $\mathrm{c}$ & Mauricio Roberto Motta Pinto da Luz & $\mathrm{d}$ \\
\hline Maria Norma Melo & $\mathrm{a}$ & Maurilio Jose Soares & $\mathrm{a}$ \\
\hline Maria Olivia Amado R Bacellar & a & Mauro Celio de Almeida Marzochi & a \\
\hline Maria Pilar Iribar Calvo & a & Mauro Ferreira de Azevedo & $\mathrm{a}$ \\
\hline Maria Regina Lima & a & Mauro Javier Cortez Veliz & $\mathrm{a}$ \\
\hline Maria Regina Reis Amendoeira & a & Mauro Shugiro Tada & $\mathrm{a}$ \\
\hline Maria Rosimery de Carvalho & e & Max Jean de Ornelas Toledo & $\mathrm{a}$ \\
\hline Maria Sandra Andrade & $\mathrm{d}$ & Mayra Xiomara Hernandez Sanabria & a \\
\hline Maria Silvia Furquim de Azevedo Morgulis & $\mathrm{c}$ & Meire Ioshie Hiyane & $\mathrm{a}$ \\
\hline Maria Stela Branquinho & a & Meire Maria de Lima & $\mathrm{d}$ \\
\hline Maria Terezinha Bahia & $\mathrm{a}$ & Melissa Orzechowski Xavier & $\mathrm{c}$ \\
\hline Maria Valdrinez Campana Lonardoni & a & Melissa Regina Fessel & $\mathrm{e}$ \\
\hline Maria Vos Paumgartten Deane & a & Michael Alexander Miles & $\mathrm{b}$ \\
\hline
\end{tabular}




\begin{tabular}{|c|c|c|c|}
\hline Name & Group & Name & Group \\
\hline Michael Waisberg & $\mathrm{c}$ & Nataly Araujo de Souza & a \\
\hline Michel Pinkus Rabinovitch & $\mathrm{a}$ & Nayara de Oliveira Belo & a \\
\hline Michelle Aparecida Ribeiro Freitas & a & Neci Matos Soares & $\mathrm{a}$ \\
\hline Michelle Barbi de Moura & - & Neide Maria da Silva & $\mathrm{a}$ \\
\hline Michelle Cristina Guerreiro dos Reis & $\mathrm{c}$ & Nelson da Silva Cordeiro & $\mathrm{c}$ \\
\hline Michelle Frazao Muzitano & a & Nelson Monteiro Vaz & $\mathrm{c}$ \\
\hline Michelle Tanny Cunha do Nascimento & a & Nelson Papavero & $\mathrm{c}$ \\
\hline Michelle Viviane Sa dos Santos & $\mathrm{c}$ & Neuza Maria Alcantara Neves & e \\
\hline Michelline do Vale Maciel & $\mathrm{e}$ & Newton Valerio Verbisck & $\mathrm{e}$ \\
\hline Milan Trsic & $\mathrm{c}$ & Ney Ribeiro Leite & $\mathrm{d}$ \\
\hline Milane de Souza Leite & e & Nicolau Maues Serra Freire & $\mathrm{c}$ \\
\hline Milden Rodrigues de Santa Rosa & - & Nildimar Alves Honorio & - \\
\hline Milena Botelho Pereira Soares & a & Nilton Barnabe Rodrigues & a \\
\hline Milena de Paiva Cavalcanti & a & Nitza Maria Alencar Gomes & $\mathrm{a}$ \\
\hline Milene Alvarenga Rachid & e & Nobuko Yoshida & $\mathrm{a}$ \\
\hline Miliane Moreira Soares de Souza & $\mathrm{c}$ & Noemi Nosomi Taniwaki & $\mathrm{a}$ \\
\hline Milton Adriano Pelli Oliveira & a & Norah Medrano Mercado & - \\
\hline Miralba Freire Carvalho Ribeiro da Silva & $\mathrm{d}$ & Norbert Miekeley & - \\
\hline Miria Gomes Pereira & a & Norma Vollmer Labarthe & $\mathrm{c}$ \\
\hline Miriam Cristina Leandro Dorta & a & Nubia Estella Matta Camacho & - \\
\hline Miriam da Silva Rocha & $\mathrm{d}$ & Octavio Fernandes da Silva Filho & $\mathrm{a}$ \\
\hline Miriam de Souza Macre & $\mathrm{d}$ & Odair Genaro & $\mathrm{a}$ \\
\hline Miriam Maria Silva Costa & - & Olavo dos Santos Pereira Junior & $\mathrm{c}$ \\
\hline Miriam Oliveira E Rocha & - & Olga Fischman Gompertz & $\mathrm{c}$ \\
\hline Miriam Silva Rafael & $\mathrm{a}$ & Olindo Assis Martins Filho & $\mathrm{a}$ \\
\hline Mirian Claudia de Souza Pereira & a & Olney Vieira da Motta & $\mathrm{c}$ \\
\hline Mirian Silva do Carmo & $\mathrm{e}$ & Osmarina Pereira da Paixao e Silva & a \\
\hline Mirko Giuliano Rojas Cortez & - & Osvaldo Andrade Santos Filho & $\mathrm{e}$ \\
\hline Mirna Aparecida Pereira & $\mathrm{c}$ & Osvaldo Marinotti & $\mathrm{a}$ \\
\hline Miryam Marroquin Quelopana & e & Osvaldo Pompilio de Melo Neto & a \\
\hline Mislaine Adriana Brezam & - & Otavio de Toledo Nobrega & e \\
\hline Moacir Paranhos Silva & $\mathrm{a}$ & Otavio Henrique Thiemann & $\mathrm{a}$ \\
\hline Moema de Alencar Hausen & e & Otilio Machado Pereira Bastos & a \\
\hline Monamaris Marques Borges & $\mathrm{a}$ & Pamela Orjuela Sanchez & $\mathrm{a}$ \\
\hline Monica Bastos de Lima Barros & $\mathrm{c}$ & Patricia Alves Morking & $\mathrm{a}$ \\
\hline Monica Cristina de Moraes Silva & $\mathrm{a}$ & Patricia Carneiro dos Santos & $\mathrm{c}$ \\
\hline Monica da Silva Nunes & $\mathrm{d}$ & Patricia Cuervo Escobar & a \\
\hline Monica de Melo Medeiros & - & Patricia de Azambuja Penna & a \\
\hline Monica Lucia Giraldo Restrepo & - & Patricia de Oliveira Camera & $\mathrm{a}$ \\
\hline Monica Lucia Gomes & $\mathrm{a}$ & Patricia Fampa Negreiros Lima & a \\
\hline Monica Maria de Albuquerque Pontes & $\mathrm{c}$ & Patricia Flavia Quaresma & a \\
\hline Monica Martinelli Nunes de Carvalho & - & Patricia Giupponi Cardoso & a \\
\hline Monique Mantovani & $\mathrm{d}$ & Patricia Irie Furuta & $\mathrm{d}$ \\
\hline Mucio Flavio Barbosa Ribeiro & a & Patricia Izquierdo Cafrune & $\mathrm{c}$ \\
\hline Myrian Morato Duarte & $\mathrm{d}$ & Patricia Karla Santos Ramos & $\mathrm{a}$ \\
\hline Myrna Cristina Bonaldo & $\mathrm{e}$ & Patricia Maria D Almeida Lima & $\mathrm{c}$ \\
\hline Myrna Garcia Serrano & e & Patricia Massara Martinelli & $\mathrm{a}$ \\
\hline Nadia Regina Pereira Almosny & a & Patricia Pellegrino de Souza & $\mathrm{c}$ \\
\hline Nadjar Nitz Silva E Silva & - & Patricia Puccinelli Orlandi Nogueira & $\mathrm{c}$ \\
\hline Naftale Katz & $\mathrm{c}$ & Patricia Regina Barboza Araujo & - \\
\hline Nagila Francinete Costa Secundino & a & Patricia Rosa de Araujo & $\mathrm{d}$ \\
\hline Nanci Akemi Missawa & $\mathrm{d}$ & Patricia Sampaio Tavares Veras & $\mathrm{a}$ \\
\hline Nanci do Nascimento & $\mathrm{c}$ & Patricia Silva Cisalpino & $\mathrm{c}$ \\
\hline Nancy Stella Vargas Bonilla & $\mathrm{d}$ & Patricia Soares de Maria de Medeiros & $\mathrm{a}$ \\
\hline Nara Amelia da Rosa Farias & a & Patricia Spoto Correa & - \\
\hline Narcisa Leal da Cunha E Silva & a & Patricio Andres Manque Manque & $\mathrm{e}$ \\
\hline Nassim da Silveira Calixto & $\mathrm{c}$ & Patricio Emilio Porcile Cerda & - \\
\hline Natal Santos da Silva & e & Patrick Abdala Fonseca Gomes & $\mathrm{d}$ \\
\hline Natalino Hagime Yoshinari & - & Paul David Stolley & $\mathrm{c}$ \\
\hline
\end{tabular}




\begin{tabular}{|c|c|c|c|}
\hline Name & Group & Name & Group \\
\hline Paula Dias Bevilacqua & $\mathrm{c}$ & Raissa Bocchi Pedroso & $\mathrm{a}$ \\
\hline Paula Lobao Antunes de Siqueira Torres & - & Raquel da Silva Pacheco & $\mathrm{a}$ \\
\hline Paula Melo de Abreu Vieira & a & Raquel Elisa da Silva Lopez & a \\
\hline Paula Rocha Moreira & $\mathrm{c}$ & Raquel Ferreira Rodrigues & a \\
\hline Paula Rogerio Fernandes & - & Raquel Lopes Martins Souza & $\mathrm{c}$ \\
\hline Paula Viviane de Souza Queiroz & - & Raquel Rodrigues Britto & $\mathrm{c}$ \\
\hline Paulo Afonso Nogueira & a & Raquel Saucier Gomes & d \\
\hline Paulo Alexandre Abrahamsohn & $\mathrm{c}$ & Raquel Silva Lisboa & $\mathrm{e}$ \\
\hline Paulo Batista de Carvalho & $\mathrm{a}$ & Raul Manhaes de Castro & $\mathrm{c}$ \\
\hline Paulo Carvalho Ferreira & $\mathrm{c}$ & Raymundo Martins de Castro & a \\
\hline Paulo Cesar Augusto de Souza & $\mathrm{a}$ & Regiane Mathias & a \\
\hline Paulo de Tarso Goncalves Leopoldo & $\mathrm{a}$ & Regina Celia Bressan Queiroz de Figueiredo & a \\
\hline Paulo de Tarso Landgraf Botteon & e & Regina Celia Lima de Macedo & $\mathrm{c}$ \\
\hline Paulo de Toledo Artigas & $\mathrm{c}$ & Regina de Fatima dos Santos Braz & a \\
\hline Paulo Eduardo Alencar de Souza & $\mathrm{c}$ & Regina Helena Saramago Peralta & a \\
\hline Paulo Eduardo Martins Ribolla & $\mathrm{a}$ & Regina Maria Barretto Cicarelli & a \\
\hline Paulo Emilio Vanzolini & $\mathrm{c}$ & Regina Maura Bueno Franco & a \\
\hline Paulo Fernando de Vargas Peixoto & $\mathrm{c}$ & Regina Mitsuka Bregano & a \\
\hline Paulo Fernando Tormin Borges Crosara & $\mathrm{c}$ & Reginaldo Pecanha Brazil & a \\
\hline Paulo Filemon Paolucci Pimenta & $\mathrm{a}$ & Regis Bernardo Brandim Gomes & a \\
\hline Paulo Henrique da Costa Pinheiro & a & Renata Ben Baisch & - \\
\hline Paulo Inacio da Costa & $\mathrm{c}$ & Renata Cristina Pardos Baida & $\mathrm{d}$ \\
\hline Paulo Nogueira Neto & $\mathrm{c}$ & Renata de Lima Alvarenga Bottrel & d \\
\hline Paulo Novis Rocha & e & Renata de Meirelles Santos Pereira & a \\
\hline Paulo Oldemar Scherer & $\mathrm{c}$ & Renata Grespan & e \\
\hline Paulo Paes de Andrade & $\mathrm{a}$ & Renata Guerra de Sa Cota & $\mathrm{c}$ \\
\hline Paulo Roberto Lima Machadoo & a & Renata Oliveira de Araujo Soares & a \\
\hline Paulo Sergio Monzani & $\mathrm{d}$ & Renata Rosito Tonelli & $\mathrm{a}$ \\
\hline Paulo Soares da Costa & $\mathrm{c}$ & Renata Santiago Alberto Carlos & $\mathrm{e}$ \\
\hline Pedro Albajar Vinas & - & Renata Tome Alves & $\mathrm{a}$ \\
\hline Pedro Geraldo Pascutti & $\mathrm{c}$ & Renata Tonhosolo & a \\
\hline Pedro Henrique Ferri & $\mathrm{c}$ & Renata Torres de Souza & a \\
\hline Pedro Lagerblad de Oliveira & $\mathrm{a}$ & Renato Arruda Mortara & $\mathrm{a}$ \\
\hline Pedro Muanis Persechini & $\mathrm{c}$ & Renato Augusto Damatta & a \\
\hline Pedro Roosevelt Torres Romao & $\mathrm{a}$ & Renato Porrozzi de Almeida & e \\
\hline Pedro Sadi Monteiro & - & Renato Sathler Avelar & a \\
\hline Pedro Silva Pinto & $\mathrm{a}$ & Ricardo Andrade Barata & $\mathrm{a}$ \\
\hline Percy Cyril Claude Garnham & $\mathrm{b}$ & Ricardo de Matos Santa Rita & - \\
\hline Peter Lydyard & - & Ricardo Goncalves & $\mathrm{a}$ \\
\hline Phileno Pinge Filho & $\mathrm{a}$ & Ricardo Lourenco de Oliveira & $\mathrm{a}$ \\
\hline Philip Davis Marsden & $\mathrm{a}$ & Ricardo Luiz Dantas Machado & - \\
\hline Philippe Dorchies & - & Ricardo Pereira Igreja & - \\
\hline Philippe Grellier & a & Ricardo Ramos Mendonca Filho & $\mathrm{c}$ \\
\hline Piero Bagnaresi & $\mathrm{a}$ & Ricardo Ribeiro dos Santos & $\mathrm{a}$ \\
\hline Pierre Grabar & - & Ricardo Santiago Gomez & $\mathrm{c}$ \\
\hline Poliana Deolindo & $\mathrm{e}$ & Ricardo Scher & $\mathrm{a}$ \\
\hline Priscila Carneiro Campos & $\mathrm{a}$ & Ricardo Toshio Fujiwara & a \\
\hline Priscila Grynberg & $\mathrm{a}$ & Ricardo Tostes Gazzinelli & $\mathrm{a}$ \\
\hline RT Damian & - & Ricardo Wagner de Almeida Vitor & a \\
\hline Rafael Dhalia & a & Ricardo Wagner Dias Portela & a \\
\hline Rafael Maciel de Freitas & $\mathrm{a}$ & Rilke Tadeu Fonseca de Freitas & $\mathrm{c}$ \\
\hline Rafael Marques Porto & $\mathrm{d}$ & Rinaldo Aparecido Mota & a \\
\hline Rafael Miyazawa Martins & $\mathrm{a}$ & Rinaldo Duarte & $\mathrm{d}$ \\
\hline Rafael Otavio Cancado Motta & $\mathrm{a}$ & Rita de Cassia Alves Alcantara de Menezes & $\mathrm{e}$ \\
\hline Rafael Paranhos de Mendonca & $\mathrm{c}$ & Rita de Cassia dos Santos Vasconcellos & $\mathrm{c}$ \\
\hline Rafael Verissimo Monteiro & a & Rita de Cassia Moreira de Souza & a \\
\hline Rafael Victorio Carvalho Guido & $\mathrm{a}$ & Rita de Cassia Pontello Rampazzo & a \\
\hline Raimunda Nonata Ribeiro Sampaio & a & Rita de Cassia Ruiz & $\mathrm{e}$ \\
\hline Raimundo Wilson de Carvalho & a & Rita do Socorro Uchoa da Silva & $\mathrm{e}$ \\
\hline
\end{tabular}




\begin{tabular}{|c|c|c|c|}
\hline Name & Group & Name & Group \\
\hline Riva de Paula Oliveira & $\mathrm{a}$ & Rosana Maria Feio Libonati & $\mathrm{c}$ \\
\hline Robert Coffman & - & Rosana Regina de Saldanha & $\mathrm{c}$ \\
\hline Roberta Dias Rodrigues Rocha & $\mathrm{a}$ & Rosane Maria Temporal & $\mathrm{a}$ \\
\hline Roberta Lemos Freire & a & Rosane Moreira Silva de Meirelles & $\mathrm{d}$ \\
\hline Roberta Olmo Pinheiro & $\mathrm{e}$ & Rosangela Clara Paulino & $\mathrm{d}$ \\
\hline Roberta Ribeiro Silva & $\mathrm{e}$ & Rosangela Locatelli Dittrich & $\mathrm{a}$ \\
\hline Roberto Carlos Grassi Malta & $\mathrm{e}$ & Rosangela Maria Rodrigues & $\mathrm{a}$ \\
\hline Roberto Esteves Pires Castanho & $\mathrm{a}$ & Rosangela Zacarias Machado & a \\
\hline Roberto Jose da Silva Badaro & $\mathrm{a}$ & Rosaura Penaranda Carrillo & - \\
\hline Roberto Jose Meyer Nascimento & $\mathrm{c}$ & Rosely Maria Conceicao Zancope & - \\
\hline Roberto Martins Goncalves & $\mathrm{c}$ & Rosiane Aparecida da Silva & a \\
\hline Roberto Mitsuyoshi Hiramoto & $\mathrm{a}$ & Rossana Correa Netto de Melo & $\mathrm{e}$ \\
\hline Roberto Montoya Araujo & - & Rossiane Claudia Vommaro & a \\
\hline Roberto Regis Magalhaes Pinto & $\mathrm{c}$ & Rozalia Mendes Figueira Campos & $\mathrm{a}$ \\
\hline Roberto Rudge de Moares Barros & a & Rubem Figueiredo Sadok Menna Barreto & $\mathrm{a}$ \\
\hline Roberto Ruhman Daher & $\mathrm{c}$ & Rubens Jose do Nascimento & $\mathrm{d}$ \\
\hline Robin G Bell & $\mathrm{c}$ & Rubens Riscala Madi & $\mathrm{c}$ \\
\hline Robson Amaro Augusto da Silva & a & Ruberval Armando Lopes & $\mathrm{c}$ \\
\hline Robson Cavalcanti Ferreira & $\mathrm{d}$ & Rute Maria Goncalves de Andrade & $\mathrm{c}$ \\
\hline Robson Coutinho Silva & $\mathrm{c}$ & Sabrina Castilho Duarte & $\mathrm{a}$ \\
\hline Robson Sartorello & $\mathrm{d}$ & Salvatore Giovanni de Simone & a \\
\hline Rodivan Braz da Silva & $\mathrm{c}$ & Samanta Etel Treiger Borborema & $\mathrm{a}$ \\
\hline Rodolfo Antonio Devera & - & Samuel Goldenberg & a \\
\hline Rodolfo Cordeiro Giunchetti & a & Samuel Pessoa & a \\
\hline Rodolfo Pereira Mendes & - & Sandra Antonia Tagliavini Santos & $\mathrm{c}$ \\
\hline Rodrigo Barbosa Ferraro & $\mathrm{d}$ & Sandra Cristina Gomes Oliveira & $\mathrm{c}$ \\
\hline Rodrigo Correa de Oliveira & a & Sandra do Lago Moraes Avila & - \\
\hline Rodrigo Costa da Silva & $\mathrm{a}$ & Sandra Lucia da Cunha E Silva & $\mathrm{c}$ \\
\hline Rodrigo Hinojosa Valdez & $\mathrm{e}$ & Sandra Mara Alessi Aristides Arraes & - \\
\hline Rodrigo Pedro Pinto Soares & $\mathrm{a}$ & Sandra Mayumi Nishi & $\mathrm{a}$ \\
\hline Roger Louis Storck & - & Sandra Regina Afonso Cardoso & $\mathrm{e}$ \\
\hline Roger T Dean & $\mathrm{b}$ & Sandro Nelson Lunedo & $\mathrm{a}$ \\
\hline Rogeria Maria Ventura & $\mathrm{d}$ & Sanivia Aparecida de Lima Pereira & $\mathrm{a}$ \\
\hline Rogerio Amino & $\mathrm{b}$ & Sanny Helena Valente de Oliveira Alberio & - \\
\hline Rogerio Costa Gondim & $\mathrm{c}$ & Santos Joao Barberino & $\mathrm{e}$ \\
\hline Rogerio Luiz Kopp & a & Santuza Maria Ribeiro Teixeira & a \\
\hline Rogerio Meneghini & $\mathrm{c}$ & Sara Timoteo Passos & $\mathrm{a}$ \\
\hline Rogerio Saad Vaz & a & Sarah Maria Singulano Cinque & - \\
\hline Roland J Terry & - & Saulo Cabral Bourguignon & a \\
\hline Romario Cerqueira Leite & $\mathrm{c}$ & Saulo Teixeira de Moura & $\mathrm{a}$ \\
\hline Romeu Cantusio Neto & $\mathrm{c}$ & Savio Amado da Silva & $\mathrm{c}$ \\
\hline Romulo Menna Barreto Valenca & $\mathrm{a}$ & Sebastiao Afonso Viana Macedo Neves & $\mathrm{c}$ \\
\hline Romulo Tadeu Dias de Oliveira & $\mathrm{c}$ & Sebastiao Aldo da Silva Valente & a \\
\hline Ronald Bastos Freire & e & Sebastiao Cronemberger Sobrinho & $\mathrm{c}$ \\
\hline Ronald Sederoff & - & Sebastiao Martins Souza Netto & - \\
\hline Ronaldo Peres Costa & $\mathrm{d}$ & Sebastiao Tostes Junior & $\mathrm{d}$ \\
\hline Ronaldo Reis & - & Selene Maia de Morais & a \\
\hline Ronaldo Ribeiro de Albuquerque & $\mathrm{c}$ & Selma Aparecida Souza Kuckelhaus & $\mathrm{c}$ \\
\hline Ronaldo Zucchi & $\mathrm{c}$ & Selma Bacellar de Souza Leao & $\mathrm{a}$ \\
\hline Ronei Luciano Mamoni & $\mathrm{c}$ & Selma Giorgio & a \\
\hline Ronize Andreia Ferreira & $\mathrm{a}$ & Selma Lilian Sallenave Salles & - \\
\hline Rosa Amelia Maldonado Medina & a & Selma Maria Bezerra Jeronimo & $\mathrm{a}$ \\
\hline Rosa Maria Esteves Arantes & $\mathrm{a}$ & Sergian Vianna Cardozo & $\mathrm{a}$ \\
\hline Rosa Maria Ferreira Noguerol Odorizzi & $\mathrm{d}$ & Sergio Caldas & $\mathrm{c}$ \\
\hline Rosa Maria Tubaki & $\mathrm{a}$ & Sergio Costa Oliveira & $\mathrm{c}$ \\
\hline Rosa Teixeira de Pinho & a & Sergio Danilo Junho Pena & $\mathrm{a}$ \\
\hline Rosalia Morais Torres & a & Sergio de Albuquerque & a \\
\hline Rosana Alves Martorelli & a & Sergio G Coutinho & a \\
\hline Rosana de Sousa Veras & a & Sergio Henrique da Silva & $\mathrm{c}$ \\
\hline
\end{tabular}




\begin{tabular}{|c|c|c|c|}
\hline Name & Group & Name & Group \\
\hline Sergio Henrique Ferreira & $\mathrm{c}$ & Susana Balmant Emerique Simoes & $\mathrm{c}$ \\
\hline Sergio Henrique Seabra & a & Suse Daise Silva Barbosa & - \\
\hline Sergio Marcelo Rodriguez Malaga & $\mathrm{a}$ & Susie Andries Nogueira & $\mathrm{c}$ \\
\hline Sergio Reyes & $\mathrm{c}$ & Suzana Elisa Moreno & - \\
\hline Sergio Salles Xavier & c & Suzana Passos Chaves & $\mathrm{a}$ \\
\hline Sergio Schenkman & a & Suzete Araujo Oliveira Gomes & $\mathrm{a}$ \\
\hline Sergio Steven Cornejo Rubin de Celis & $\mathrm{e}$ & Sylvia Regina Temer Cursino & $\mathrm{d}$ \\
\hline Sergio Verajovski Almeida & $\mathrm{c}$ & Sylvio Celso Goncalves da Costa & a \\
\hline Sheila Cristina Nardelli & $\mathrm{a}$ & Symone Fulgencio Lima & $\mathrm{c}$ \\
\hline Sheila Tavares Pereira & $\mathrm{d}$ & Taciana de Figueiredo Soares & $\mathrm{c}$ \\
\hline Shirley Maddison & - & Taia Maria Berto Rezende & e \\
\hline Siegfried Siegesmund & - & Tais Nobrega de Sousa & $\mathrm{a}$ \\
\hline Silvana Maria Eloi Santos & $\mathrm{a}$ & Tamara Nunes de Lima Camara & $\mathrm{a}$ \\
\hline Silvana Marques de Araujo & a & Tania Cremonini de Araujo Jorge & $\mathrm{a}$ \\
\hline Silvana Tulio Fortes & $\mathrm{c}$ & Tania Mara Pinto Dabes Guimaraes & $\mathrm{e}$ \\
\hline Silvane Maria Fonseca Murtat & a & Tania Maria Pacheco Shubach & - \\
\hline Silvia Amaral Goncalves da Silva & $\mathrm{a}$ & Tania Maria Valente Pacheco & $\mathrm{c}$ \\
\hline Silvia Beatriz Boscardin & a & Tania Santos de Marco & - \\
\hline Silvia Cristina Osaki & $\mathrm{c}$ & Tania Ueda Nakamura & $\mathrm{a}$ \\
\hline Silvia de Barros Mazon & $\mathrm{e}$ & Tania Zaverucha do Valle & $\mathrm{a}$ \\
\hline Silvia Ermelinda Barbosa & a & Tanos Celmar Costa Franca & $\mathrm{e}$ \\
\hline Silvia Maria Santos Carvalho & $\mathrm{a}$ & Tatiana Berber Teixeira & $\mathrm{d}$ \\
\hline Silvia Maria Spalding & $\mathrm{a}$ & Tatiana Galvao de Melo de Oliveira & $\mathrm{a}$ \\
\hline Silvia Reni Bortolin Uliana & $\mathrm{a}$ & Tatiana Rodrigues de Moura & $\mathrm{a}$ \\
\hline Silvio Bertini & $\mathrm{d}$ & Tatiana Shioji Tiuman & $\mathrm{a}$ \\
\hline Silvio Celso Gomes da Costa & - & Tatiane Luciano Balliano & $\mathrm{c}$ \\
\hline Silvio Favoreto Junior & $\mathrm{d}$ & Tatjana Keesen de Souza Lima & $\mathrm{a}$ \\
\hline Silvio Luis Pereira de Souza & $\mathrm{a}$ & Tecia Maria Ulisses de Carvalho & $\mathrm{a}$ \\
\hline Silvio Santana Dolabella & a & Tercio de Souza Goes & - \\
\hline Simone Aparecida Antoniazi & $\mathrm{d}$ & Teresa Cristina Bergamo do Bomfim & a \\
\hline Simone Cardoso Leon & $\mathrm{a}$ & Teresa Cristina Franca de Assumpcao & a \\
\hline Simone da Fonseca Pires & $\mathrm{a}$ & Teresa Cristina Leandro de Jesus & $\mathrm{a}$ \\
\hline Simone Ladeia Andrade & a & Teresa Cristina Melo Garcia & $\mathrm{c}$ \\
\hline Simone Patricia Carneiro de Freitas & $\mathrm{a}$ & Teresa Cristina Monte Goncalves & a \\
\hline Simone Pontes Xavier & $\mathrm{c}$ & Teresa Fernandes Silva do Nascimento & $\mathrm{a}$ \\
\hline Simoni Machado de Medeiros & $\mathrm{d}$ & Tereza Cristina Orlando & $\mathrm{a}$ \\
\hline Sineide Maria de Oliveira Vilela & - & Thais Cristina Baeta Soares Souto Padron & a \\
\hline Sinval Pinto Brandao Filho & a & Thais Ferreira Isabel & a \\
\hline Solange Cristina Uber Busek & $\mathrm{c}$ & Thais Gomes Verzignassi Silveira & $\mathrm{a}$ \\
\hline Solange do Perpetuo Socorro Evangelista Costa & $\mathrm{c}$ & Thais Helena Constantino Patelli & $\mathrm{e}$ \\
\hline Solange Lisboa de Castro & a & Thales de Brito & a \\
\hline Solange Maria Gennari & $\mathrm{c}$ & Thatiane Santos de Simone & - \\
\hline Solange Maria Magalhaes da Silva Porto & $\mathrm{c}$ & Thiago de Castro Gomes & a \\
\hline Sonia de Lucena Mioranza & a & Thiago Santana Gastardelo & $\mathrm{d}$ \\
\hline Sonia Gumes Andrade & a & Thuioshi Ioneda & $\mathrm{c}$ \\
\hline Sonia Jancar & $\mathrm{c}$ & Tiago Moreno Castilho & $\mathrm{a}$ \\
\hline Sonia Maria de Freitas & $\mathrm{c}$ & Tiago Wilson Patriarca Mineo & $\mathrm{a}$ \\
\hline Sonia Maria Neumann Cupolilo & a & Tom Blundell & $\mathrm{b}$ \\
\hline Sonia Nair Bao & $\mathrm{c}$ & Tomaz Aroldo da Mota Santos & $\mathrm{c}$ \\
\hline Sonia Regina Lambert Passos & $\mathrm{c}$ & Tonny Hiroshi Katsuragawa & - \\
\hline Sonia Rolim Reis & a & Tricia Maria Ferreira de Sousa Oliveira & $\mathrm{a}$ \\
\hline Sonia Soares Costa & a & Tulio Marcos Santos & $\mathrm{c}$ \\
\hline Soraya Torres Gaze & $\mathrm{e}$ & Ubirajara Ribeiro Martins de Souza & $\mathrm{c}$ \\
\hline Stefano Trapani & $\mathrm{a}$ & Udelysses Janete Veltrini Fonzar & $\mathrm{c}$ \\
\hline Stella Maria Barrouin Melo & a & Ueslei Teodoro & $\mathrm{d}$ \\
\hline Stella Maris Gonzalez Cappa & $\mathrm{b}$ & Ulisses Gazos Lopes & $\mathrm{a}$ \\
\hline Stenio Perdigao Fragoso & a & Uriel Franco da Rocha & a \\
\hline Steven Reed & $\mathrm{b}$ & Usha Vashist & $\mathrm{b}$ \\
\hline Sthenia Santos Albano Amora & $\mathrm{c}$ & Vaca Marin Miguel Angel & - \\
\hline Stuart Schlossman & - & Vagner Jose Mendonca & a \\
\hline Sueli Fumie Yamada Ogatta & a & Valdelaine Etelvina Miranda de Araujo & $\mathrm{d}$ \\
\hline Susana Andrea Sculaccio & d & Valdelice Oliveira Burgos & $\mathrm{d}$ \\
\hline
\end{tabular}




\begin{tabular}{|c|c|c|c|}
\hline Name & Group & Name & Group \\
\hline Valderez Bastos Valero Lapchik & $\mathrm{e}$ & Wagner Campos Otoni & $\mathrm{c}$ \\
\hline Valdir de Queiroz Balbino & a & Wagner Loyola & $\mathrm{c}$ \\
\hline Valeria Cavalcanti Rolla & $\mathrm{c}$ & Wagner Luiz Tafuri & $\mathrm{a}$ \\
\hline Valeria Marcal Felix de Lima & $\mathrm{a}$ & Wagner Welber Arrais da Silva & $\mathrm{a}$ \\
\hline Valeria Pereira Nacife & $\mathrm{e}$ & Wagnner Jose Nascimento Porto & e \\
\hline Valeria Rego Alves Pereira & - & Waldemar Naves do Amaral & e \\
\hline Valeska de Andrade Stempliuk & $\mathrm{e}$ & Walderez Ornelas Dutra & $\mathrm{a}$ \\
\hline Valter da Silva Queiroz & $\mathrm{c}$ & Wallace Raimundo Araujo dos Santos & $\mathrm{c}$ \\
\hline Valter Ferreira de Andrade Neto & $\mathrm{a}$ & Walter Belda Junior & $\mathrm{c}$ \\
\hline Vanderlei Rodrigues & $\mathrm{c}$ & Walter Ceretti Junior & a \\
\hline Vanessa Cabreira de Freitas & $\mathrm{a}$ & Walter Colli & $\mathrm{a}$ \\
\hline Vanessa Diniz Atayde & - & Walter dos Santos Lima & $\mathrm{c}$ \\
\hline Vanessa Migliaccio & $\mathrm{a}$ & Walter Fiers & - \\
\hline Vanessa Muradian & - & Walter Flausino & $\mathrm{a}$ \\
\hline Vanessa Peruhype Magalhaes Pascoal & a & Walter Kindro Andreoli & $\mathrm{d}$ \\
\hline Vanessa Santos Sotomaior & e & Walter Leira Teixeira Filho & $\mathrm{d}$ \\
\hline Vanessa Silvestre Ferreira de Oliveira & - & Wander Rogerio Pavanelli & $\mathrm{a}$ \\
\hline Vanete Thomaz Soccol & a & Wanderley de Souza & $\mathrm{a}$ \\
\hline Vania Lucia Brandao Nunes & $\begin{array}{c}\mathrm{a} \\
\mathrm{a}\end{array}$ & Wanderli Pedro Tadei & $\mathrm{a}$ \\
\hline Vania Lucia de Assis Santana & a & Wanderson Duarte da Rocha & $\mathrm{a}$ \\
\hline Vania Rita Elias Pinheiro Bittencourt & c & Wanderson Geraldo de Lima & $\mathrm{a}$ \\
\hline $\begin{array}{l}\text { Vania Rita Elias Pinheiro Bittencourt } \\
\text { Vanize de Oliveira Macedo }\end{array}$ & $\mathrm{c}$ & Wanessa Christina de Souza Neiras & $\mathrm{d}$ \\
\hline $\begin{array}{l}\text { Vanize de Oliveira Macedo } \\
\text { Vania Maria Veloso }\end{array}$ & a & Warren Thomas Atyeo & - \\
\hline Vanja Maria Veloso & d & Washington Luis Conrado dos Santos & $\mathrm{a}$ \\
\hline Vanja Suely Pachiano Calvosa & - & Washington Luiz Tafuri & $\mathrm{a}$ \\
\hline Vera da Costa Valente & a & Wayner Vieira de Souza & $\mathrm{c}$ \\
\hline Vera Lucia Costa Vale & $\mathrm{c}$ & Welber Daniel Zanetti Lopes & $\mathrm{c}$ \\
\hline Vera Lucia Pereira Chioccola & a & Wendel Coura Vital & $\mathrm{a}$ \\
\hline Vera Margareth Scarpassa & - & Wendell Sergio Ferreira Meira & a \\
\hline Vera Silvia de Freitas Vinhais & - & Wilhelm Otto Daniel Martin Neitz & - \\
\hline Veronica Figueiredo do Amaral & a & Wilia Marta Elsner Diederichsen de Brito & $\mathrm{c}$ \\
\hline Veronica Gomes Monteiro & $\mathrm{e}$ & William A Ayer & - \\
\hline Veronica Schmitz Pereira & a & William Mello Kattenbach & $\mathrm{a}$ \\
\hline Vicente Amato Neto & $\mathrm{c}$ & Williams Jorge da Cruz Macedo & $\mathrm{a}$ \\
\hline Vicente de Paula Antunes Teixeira & $\mathrm{c}$ & Willian de Castro Borges & - \\
\hline Vicente de Paulo Coelho Peixoto de Toledo & a & Wilmar Dias da Silva & $\mathrm{c}$ \\
\hline Victor Martin Quintana Flores & $\mathrm{c}$ & Wilson Duarte Alecrim & - \\
\hline Victor Nussenzweig & $\mathrm{b}$ & Wilson Jacinto Silva de Souza & a \\
\hline Victorio Valeri & - & Wilson Roberto Navega Lodi & $\mathrm{c}$ \\
\hline Vinicius Cotta de Almeida & e & Wilson Savino & a \\
\hline Vinicius Santana Nunes & a & Wilton Carlos Zanetti Lopes & - \\
\hline Virginia Maria Barros de Lorena & a & Wim Maurits Sylvain Degrave & $\mathrm{a}$ \\
\hline Virginia Maria Goes da Silva & a & Wladimir Lobato Paraense & a \\
\hline Virmondes Rodrigues Junior & a & Wornei Silva Miranda Braga & $\mathrm{c}$ \\
\hline Vitorino Modesto dos Santos & $\mathrm{d}$ & Yara de Miranda Gomes & a \\
\hline Viviane de Oliveira Vasconcelos & $\mathrm{c}$ & Yara Maria Traub Cseko & $\mathrm{a}$ \\
\hline Viviane Ferraz de Paula & $\mathrm{c}$ & Yasmine Belkaid & $\mathrm{b}$ \\
\hline Viviane Monteiro Goes & $\mathrm{d}$ & Zander Barreto de Miranda & - \\
\hline Viviane Rodrigues Esperandim & a & Zelia Maria Profeta Da Luz & $\mathrm{c}$ \\
\hline Viviane Sampaio Boaventura de Oliveira & a & Zelia Profeta da Luz & a \\
\hline Vladimir Michailowsky Leite Ribeiro & a & Zelson Giacomo Loss & $\mathrm{a}$ \\
\hline Vladimir Yong Gonzalez & - & Zenon Rodriguez Batista & $\mathrm{c}$ \\
\hline Vlaudia Maria Assis Costa & $\mathrm{a}$ & Zigman Brener & $\mathrm{a}$ \\
\hline Wagner Barbosa Dias & $\mathrm{e}$ & Zoilo Pires de Camargo & $\mathrm{c}$ \\
\hline
\end{tabular}

a: scientists working or who worked in the past in protozoology in Brazil; b: scientists working in protozoology outside of Brazil that were advisors of Brazilians; c: scientists that did not work in protozoology during their $\mathrm{PhD}$ or establish a research interest in this field but were advisors of scientists that migrated to protozoology; $\mathrm{d}$ : persons who developed their $\mathrm{PhD}$ in protozoology but are now involved in activities other than science; e: scientists who developed their PhD in protozoology but established research in another field. 
TABLE IV

Classification of names that developed (1) or not (2) $\mathrm{PhD}$ in protozoology

\begin{tabular}{|c|c|c|c|}
\hline Name & $1 / 2$ & Name & $1 / 2$ \\
\hline Adrian Martin Pohlit & 2 & Andre Nobrega Pitaluga & 1 \\
\hline Adriana Barrinha Fernandes & 1 & Andréa Alice da Silva & 1 \\
\hline Adriana Jardim de Almeida & 1 & Andrea Aparecida Morais Fernandes de Freitas & 1 \\
\hline Adriana Oliveira Costa & 1 & Andréa Bouer & 1 \\
\hline Adriana Oliveira dos Santos & 1 & Andrea Caetano da Silva & 1 \\
\hline Adriane Pimenta da Costa-Val Bicalho & 1 & Andrea Carla Leite Chaves & 1 \\
\hline Adriane Regina Todeschini & 1 & Andrea Cristina Vetö Arnholdt & 1 \\
\hline Adriano Defini Andricopulo & 2 & Andrea Henriques Pons & 1 \\
\hline Adriano Gomes da Silva & 1 & Andrea Mara Macedo & 1 \\
\hline Alba Valéria Machado da Silva & 1 & Andréa Pereira de Souza & 1 \\
\hline Alda Maria da Cruz & 1 & Andrea Rodrigues Avila & 1 \\
\hline Alda Maria Teixeira Ferreira & 1 & Andrea Sobral & 1 \\
\hline Aldina Maria Prado Barral & 1 & Andréa Teixeira de Carvalho & 2 \\
\hline Alejandra Saori Araki & 1 & Andrés Jimenez Galisteo Jr & 1 \\
\hline Alejandro Miguel Katzin & 1 & Andrey José de Andrade & 1 \\
\hline Alessandra D'almeida Filardy & 2 & Andreza Pain Marcelino & 1 \\
\hline Alessandra Gutierrez de Oliveira & 1 & Angela Cristina Verissimo Junqueira & 1 \\
\hline Alessandra Mara Alves Ragozo & 1 & Angela Cristina Volpini & 1 \\
\hline Alessandra Scofield & 1 & Angela Hampshire de Carvalho Santos Lopes & 1 \\
\hline Alessandro Aparecido Rodrigues da Silva & 1 & Angela Kaysel Cruz & 2 \\
\hline Alexander Sibajev & 1 & Anibal Antonio da Silva Pereira & 2 \\
\hline Alexandre Afranio Peixoto & 2 & Anita Hilda Straus Takahashi & 2 \\
\hline Alexandre Barbosa Reis & 1 & Annamaria Ravara Vago & 1 \\
\hline Alexandre Morrot Lima & 1 & Antonia Maria Ramos Franco Pereira & 1 \\
\hline Alfredo Carlos Rodrigues de Azevedo & 1 & Antoniana Ursine Krettli & 1 \\
\hline Alfredo José Altamirano-Enciso & 1 & Antonio de Oliveira Lima & 2 \\
\hline Aline Cardoso Caseca Volotão & 1 & Antonio Florêncio de Figueiredo & 2 \\
\hline Aline Chaves Alexandrino & 1 & Antonio Marcos Guimarães & 1 \\
\hline Allan Kardec Ribeiro & 1 & Antonio Raimundo Lima Cruz Teixeira & 1 \\
\hline Aluízio Prata & 2 & Antonio Walter Ferreira & 1 \\
\hline Alvaro Augusto Ribeiro D'almeida Couto & 1 & Aparecida de Fatima Michelin & 1 \\
\hline Alvaro Jose Romanha & 1 & Arlei Marcili & 1 \\
\hline Amanda Fortes Francisco & 1 & Arlene de Jesus Mendes Caldas & 1 \\
\hline Amilcar Vianna Martins Filho & 2 & Armando de Oliveira Schubach & 1 \\
\hline Ana Acacia de Sa Pinheiro & 1 & Artur Torres Cordeiro & 1 \\
\hline Ana Carolina Campi Azevedo & 2 & Astolpho Ferraz de Siqueira & 2 \\
\hline Ana Carolina de Aguiar Vasconcelos Carneiro & 1 & Augusto Mello Simões Barbosa & 1 \\
\hline Ana Cristina Bahia Nascimento & 1 & Bárbara Cruz Tavares de Macedo Fernandes & 1 \\
\hline Ana Cristina da Silva Pinto & 2 & Bartira Rossi Bergmann & 1 \\
\hline Ana Cristina de Carvalho Botelho & 1 & Beatriz Dolabela de Lima & 2 \\
\hline Ana Cristina Vianna Mariano da Rocha Lima & 1 & Beatriz Gomes Brazil & 1 \\
\hline Ana Lucia Abreu Silva & 1 & Beatriz Gomes Guimarães & 1 \\
\hline Ana Lúcia Teles Rabello & 2 & Beatriz Lilian da Silva Costa Souza & 1 \\
\hline Ana Maria de Castro & 1 & Beatriz Rossetti Ferreira & 2 \\
\hline Ana Maria Jansen-Franken & 1 & Bernardo Galvão Castro Filho & 1 \\
\hline Ana Maria Revorêdo da Silva Ventura & 1 & Bianca Brand Ederli & 1 \\
\hline Ana Paula Cabral de Araujo Lima & 1 & Bianca Perdigão Olivieri & 1 \\
\hline Ana Paula Maia Peixoto Marino & 1 & Bianca Silvana Zingales & 2 \\
\hline Ana Paula Rocha Gadelha & 1 & Blima Fux & 1 \\
\hline Ana Paula Salles Moura Fernandes & 1 & Bruno Antonio Marinho Sanchez & 1 \\
\hline Ana Yeci das Neves Pinto & 1 & Bruno da Rocha Azevedo & 1 \\
\hline Anderson Barbosa de Moura & 1 & Bruno de Bezerril Andrade & 1 \\
\hline Anderson Sena & 1 & Bruno Pereira Berto & 1 \\
\hline André Gustavo Tempone Cardoso & 1 & Camila Augusta de Oliveira Martins Arakaki & 1 \\
\hline André Luiz Fonseca de Souza & 1 & Camila Indiani de Oliveira & 1 \\
\hline André Luiz Pedrosa & 1 & Camila Marques Adade & 1 \\
\hline André Luiz Rodrigues Roque & 1 & Carina Elisei de Oliveira & 1 \\
\hline
\end{tabular}




\begin{tabular}{|c|c|c|c|}
\hline Name & $1 / 2$ & Name & $1 / 2$ \\
\hline Carina Margonari de Souza & 1 & Claudio Vieira da Silva & 1 \\
\hline Caris Maroni Nunes & 1 & Clea Andrade Chiari & 1 \\
\hline Carla Domingues dos Santos & 1 & Clea Nazare Carneiro Bichara & 2 \\
\hline Carla Mônica Pinheiro & 1 & Cleusa Alves Theodoro Rodrigues & 1 \\
\hline Carlos Alberto Pereira Tavares & 2 & Clever Gomes Cardoso & 1 \\
\hline Carlos Capistrano Gonçalves de Oliveira & 1 & Colete Fonseca & 1 \\
\hline Carlos Chagas & 2 & Conceiçao Ribeiro da Silva Machado & 2 \\
\hline Carlos Eduardo Almeida & 1 & Constança Felícia de Paoli de Carvalho Britto & 1 \\
\hline Carlos Eduardo Maia Gomes & 1 & Cristiana Ferreira Alves de Brito & 2 \\
\hline Carlos Eduardo Pereira Corbett & 2 & Cristiane Varella Lisboa & 1 \\
\hline Carlos Eduardo Tadokoro & 1 & Cristina de Souza Chaves & 1 \\
\hline Carlos Eduardo Tosta da Silva & 1 & Cristina Henriques & 1 \\
\hline Carlos Eugênio Cavasini & 1 & Cristina Wide Pissetti & 1 \\
\hline Carlos Gustavo Regis da Silva & 1 & Daniel Claudio de Oliveira Gomes & 1 \\
\hline Carlos José de Carvalho Pinto & 1 & Daniela de Melo Resende & 1 \\
\hline Carlos Luiz Massard & 1 & Daniela de Pita Pereira & 1 \\
\hline Carlos Mauricio de Figueiredo Antunes & 2 & Daniela de Stefani Marquez & 1 \\
\hline Carlos Medicis Morel & 2 & Daniela Fiori Gradia & 1 \\
\hline Carlos Noriyuki Kaneto & 1 & Daniela Luz Ambrosio & 1 \\
\hline Carlos Renato Machado & 2 & Daniela Parada Pavoni & 1 \\
\hline Carlos Wilson Gomes Lopes & 2 & Daniela Rodrigues de Faria Barbosa & 1 \\
\hline Carolina Borsoi Moraes & 1 & Daniele dos Santos Andrade & 1 \\
\hline Carolina de Oliveira Mendes Aguiar & 1 & Daniele Pereira de Castro & 1 \\
\hline Carolina Furtado Torres da Silva & 1 & Daniella Alchaar D’avila & 1 \\
\hline Carolina Guilherme Prestes Beyrodt & 1 & Daniella Castanheira Bartholomeu & 1 \\
\hline Carolina Nascimento & 1 & Daniella Regina Arantes Martins & 1 \\
\hline Caroline Dantas de Oliveira & 1 & Danielle Ferreira de Magalhães Soares & 1 \\
\hline Catarina Gadelha & 1 & Danielle Maria Nascimento Moura & 1 \\
\hline Cecilia Beatriz Fiuza Favali & 1 & Danielle Pereira Cavalcanti & 1 \\
\hline Ceclie Maria Antunes Barbosa Biancardi & 1 & Danielle Pereira Vieira & 1 \\
\hline Celene Maria de Oliveira Simões Alves & 1 & Danielle Reis Napolitano & 1 \\
\hline Celeste da Silva Freitas de Souza & 1 & Danilo Ciccone Miguel & 1 \\
\hline Celia Maria Ferreira Gontijo & 1 & Débora Pereira Garcia Melo & 2 \\
\hline Celia Maria Silva Pedrosa & 1 & Deboraci Brito Prates & 1 \\
\hline Célia Maria Vieira Vendrame & 1 & Deise Aparecida de Oliveira Silva & 1 \\
\hline Célia Regina da Silva Garcia & 2 & Deivid Costa Soares & 1 \\
\hline Célio Geraldo Freire de Lima & 1 & Denise Barcante Castro Pinto & 1 \\
\hline Celso Cruz Tavares & 1 & Denise Bertulucci Rocha Rodrigues & 1 \\
\hline Celso Vataru Nakamura & 1 & Denise Borges dos Santos Dias & 1 \\
\hline Celuta Sales Alviano & 2 & Denise da Silveira Lemos & 2 \\
\hline Cezar Martins de Sá & 2 & Denise Fonseca Córtes & 1 \\
\hline Christian Macagnan Probst & 1 & Denise Valle & 1 \\
\hline Chung Man Chin & 1 & Diego Peres Alonso & 1 \\
\hline Cicero Brasileiro de Mello Neto & 1 & Dílvia Ferreira da Silva & 1 \\
\hline Cinthia Furst Leroy Gomes Bueloni & 1 & Dulce Helena Ferreira de Souza & 1 \\
\hline Cyntia Fernandes de Souza & 1 & Edelberto Santos Dias & 1 \\
\hline Clara Lúcia Barbiéri Mestriner & 1 & Eden Ribeiro Freire & 1 \\
\hline Clarissa Romero Teixeira & 1 & Edésio José Tenório de Melo & 1 \\
\hline Claude Pirmez & 1 & Edgar Marcelino de Carvalho Filho & 1 \\
\hline Cláudia Alves de Andrade-Coelho & 1 & Edilene Alcantara de Castro & 1 \\
\hline Claudia Ida Brodskyn & 1 & Edilene Oliveira da Silva & 1 \\
\hline Claudia Magalhaes Calvet Alvarez & 1 & Edmundo Carlos Grisard & 1 \\
\hline Claudia Maia Brigagão & 1 & Edna Barbosa de Souza & 1 \\
\hline Claudia Mara Lara Melo Coutinho & 1 & Edna Freymuller Haapalainen & 2 \\
\hline Claudia Maria Leal Bevilaqua & 2 & Edson Roberto da Silva & 1 \\
\hline Claudia Martins Carneiro & 1 & Edson Rondinelli & 2 \\
\hline Claudio Roberto Madruga & 1 & Eduardo Caio Torres dos Santos & 1 \\
\hline Claudio Romero Farias Marinho & 1 & Eduardo de Almeida Marques da Silva & 1 \\
\hline Claudio Tadeu Daniel-Ribeiro & 2 & Eduardo Fonseca Pinto & 1 \\
\hline
\end{tabular}




\begin{tabular}{|c|c|c|c|}
\hline Name & $1 / 2$ & Name & $1 / 2$ \\
\hline Eduardo Henrique Gomes Rodrigues & 1 & Ferdinan Almeida Melo & 1 \\
\hline Eduardo Lani Volpe da Silveira & 1 & Fernanda Barbosa Nogueira Raad & 1 \\
\hline Eduardo Sergio da Silva & 1 & Fernanda Fortes de Araújo & 1 \\
\hline Edward Felix Silva & 1 & Fernanda Maria Santiago & 1 \\
\hline Edwards Frazão-Teixeira & 1 & Fernando Antonio Botoni & 1 \\
\hline Egler Chiari & 1 & Fernando Araujo Monteiro & 1 \\
\hline Elaine Soares Coimbra & 1 & Fernando de Pilla Varotti & 1 \\
\hline Elenice Moreira Lemos & 1 & Fernando Dias de Avila Pires & 2 \\
\hline Eliame Mouta Confort & 1 & Fernando Pavao & 1 \\
\hline Eliana Lucia Tomaz do Nascimento & 1 & Filipe Dantas-Torres & 1 \\
\hline Eliane de Morais Teixeira & 1 & Firmino Torres de Castro & 2 \\
\hline Eliane Lages Silva & 1 & Flávia Guimarães Rodrigues & 1 \\
\hline Eliane Perlatto Moura & 1 & Flavia Regina Almeida Campos Naief Moreira & 1 \\
\hline Elieth Afonso de Mesquita & 1 & Flavio Alves Lara & 1 \\
\hline Elisa Cupolillo & 1 & Flor Ernestina Martinez Espinosa & 1 \\
\hline Elisa Neves Vianna & 1 & Francisco Acácio Alves & 1 \\
\hline Elisa San Martin Mouriz Savani & 1 & Francisco Carlos Rodrigues de Oliveira & 1 \\
\hline Elizabeth Castro Moreno & 1 & Francisco de Carvalho Dias Filho & 1 \\
\hline Elizabeth de Souza Neves & 1 & Francisco Juarez Ramalho Pinto & 2 \\
\hline Elizabeth Ferreira Rangel & 1 & Franco Claudio Bonetti & 1 \\
\hline Elizabeth Gloria Oliveira Barbosa Santos & 1 & Franklin Barbalho Magalhaes & 1 \\
\hline Elizabeth Igne Ferreira & 1 & Frederico Guilherme Coutinho Abath & 2 \\
\hline Elmo Eduardo de Almeida Amaral & 1 & Gabriel Antonio Nogueira Nascentes & 1 \\
\hline Eloi de Souza Garcia & 1 & Gabriel Eduardo Melim Ferreira & 1 \\
\hline Eloisa Amélia Vieira Ferro & 1 & Gabriel Grimaldi Filho & 1 \\
\hline Eloy Guillermo Castellon Bermudez & 2 & Geisa Baptista Barros & 1 \\
\hline Elvira Maria Saraiva Chequer Bou Habib & 1 & George Alexandre dos Reis & 2 \\
\hline Elza Ferreira Noronha & 1 & George Régo Albuquerque & 1 \\
\hline Emilia Akemi Shiraishi Kimura & 1 & Georgia Correa Atella & 1 \\
\hline Eneida César Mastrantonio & 1 & Gerhard Wunderlich & 2 \\
\hline Eric Faudry & 1 & Gerson Salay & 1 \\
\hline Erica Araujo Mendes & 1 & Giani França Santoro & 1 \\
\hline Érica dos Santos Martins Duarte & 1 & Ginelza Peres Lima dos Santos & 1 \\
\hline Erica Paes Barreto Xavier Moraes & 1 & Girley Francisco Machado de Assis & 1 \\
\hline Erich Loza Telleria & 1 & Gisele Fernanda Assine Picchi & 1 \\
\hline Erick Vaz Guimarães & 1 & Giselle Maria Rachid Viana & 1 \\
\hline Erika Izumi & 1 & Gisléine Aparecida Martins & 1 \\
\hline Erika Martins Braga & 1 & Glaucius Oliva & 1 \\
\hline Erika Michalsky Monteiro & 1 & Glenda Meira Cardoso & 1 \\
\hline Erney Felicio Plessmann de Camargo & 2 & Gloria Ester Cadavid Restrepo & 1 \\
\hline Esteban Mauricio Cordero Veas & 1 & Gloria Regina Franco & 2 \\
\hline Eufrosina Setsu Umezawa & 1 & Graziela Maria Zanini & 1 \\
\hline Eunice Aparecida Bianchi Galati & 1 & Guido Fontgalland Coelho Linhares & 1 \\
\hline Evander de Jesus Oliveira Batista & 1 & Guilherme Costa Matsutani & 1 \\
\hline Eveline Gomes Vasconcelos & 2 & Guilherme Loureiro Werneck & 1 \\
\hline Evelyn Kety Pratt Riccio & 1 & Gustavo Coutinho Cerqueira & 1 \\
\hline Fabiana Rodrigues dos Santos & 1 & Gustavo Fontes Paz & 1 \\
\hline Fabiana Simão Machado & 1 & Gustavo Henrique Goulart Trossini & 1 \\
\hline Fabiane Matos dos Santos & 1 & Gustavo Miranda Rocha & 1 \\
\hline Fabiano Borges Figueiredo & 1 & Hatisaburo Masuda & 2 \\
\hline Fabio Trindade Maranhão Costa & 1 & Hector Barrabin & 2 \\
\hline Fabíola Barbieri Holetz & 1 & Heitor Franco de Andrade Junior & 1 \\
\hline Fabíola Cardillo & 1 & Heitor Miraglia Herrera & 1 \\
\hline Fabrício César Dias & 1 & Helcileia Dias Santos & 1 \\
\hline Fabricio Klerynton Marchini & 1 & Helder Magno Silva Valadares & 1 \\
\hline Falqueto Aloisio & 1 & Helen Rodrigues Martins & 1 \\
\hline Fatima Soares Motta Noronha & 1 & Helena Hilomi Taniguchi & 1 \\
\hline Felipe de Almeida Dias & 1 & Helena Lúcia Carneiro Santos & 1 \\
\hline Felipe de Mello Vigoder & 1 & Helene Santos Barbosa & 1 \\
\hline
\end{tabular}




Name $1 / 2$

Helio Langoni

Name

Henio Godeiro Lacerda

Herbert Leonel de Matos Guedes

Hernando Antonio del Portillo Obando

Hertha Meyer

Hilda Fátima de Jesus Pena

Hiro Goto

Hooman Momen

Hugo Caire de Castro Faria Neto

Humberto de Araujo Rangel

Idessania Nazareth da Costa

Igor de Almeida Rodrigues

Ilea Brandao Rodrigues

Ingrid Solange Evans Osses

Irene da Silva Soares

Isaac Roitman

Isabel Cristina Fábregas Bonna

Isabel Martinez

Isabela Penna Ceravolo

Ises de Almeida Abrahamsohn

Isis Fernandes Magalhaes Santos

Italmar Teodorico Navarro

Italo Rodrigues de Araujo Sherlock

Ivana Nunes Gomes de Araujo

Ivete Conchon Costa

Ivo Santana Caldas

Izaltina Silva Jardim Cavalli

Izilda Curado

Jacenir Reis dos Santos Mallet

Jaime Martins de Santana

Jair Pereira da Cunha Junior

Jairo Dias Barreira

Jan Carlo Morais Oliveira Bertassoni Delorenzi

Jane Lima dos Santos

Janete Soares Coelho dos Santos

Jayme Angluster

Jayme Augusto de Souza-Neto

Jayme Neves

Jeffrey Jon Shaw

Jeronimo Conceição Ruiz

Joanna Darc Aparecida Herzog Soares

João Carlos Araujo Carreira

João Carlos Pinto Dias

João Luis Garcia

João Luiz Mendes Wanderley

João Roberto da Mata

João Santana da Silva

Joaquin Hernan Patarroyo Salcedo

Jorge Clarencio Souza Andrade

Jorge Elias Kalil Filho

José Adail Fonseca de Castro

José Ailton da Silva

José Almir Moraes da Rocha

José Angelo Lauletta Lindoso

José Bento Pereira Lima

José Carlos Miranda

José Clóvis do Prado Júnior

José da Rocha Carvalheiro

Jose Daniel Figueroa-Villar

1

1

2

2

1

1

1

2

2
2

1

1

1

1

1

1

1

1

1

1

1

1

1

1

1

1

1

1

1

1

2

2

2
1

1

2

1

1

1

1

1

1

1

1

1

2

1
2

2
1

1

1

1
José Eduardo Tolezano

José Franco da Silveira Filho

José Henrique Maia Campos de Oliveira

Jose Jurberg

José Luciano Nepomuceno da Silva

Jose Manuel Macario Rebelo 2

José Marcelo Ramalho Ortigão 1

Jose Maria Alvarez Mosig 2

José Maria de Souza 2

Jose Maria Soares Barata

Jose Mauro Peralta 2

Jose Osvaldo Previato 2

Jose Ribamar Mesquita Teixeira 1

Jose Roberto Meyer Fernandes 2

Jose Roberto Mineo 1

Jose Rodrigues Coura 1

Jose Rodrigues da Silva 2

Jose Ronnie Carvalho de Vasconcelos $\quad 1$

Joseli de Oliveira Ferreira 1

Joseli Lannes-Vieira 2

Juan Miguel Villalobos-Salcedo 1

Judith Kardos Klotzel 1

Julia Pinheiro Chagas da Cunha 1

Juliana de Assis Silva Gomes Estanislau 1

Juliana de Meis 1

Juliana Dias Costa Roque

Juliana Gonzaga de Oliveira

Juliana Lopes Rangel Fietto

Juliana Perrone Bezerra de Menezes

Juliano Simões de Toledo

Juliany Cola Fernandes Rodrigues

Julio Pudles

Julio Scharfstein

Junia de Sousa Franco

Karen Angelica Cavassani de Souza

Karen dos Santos Charret

Karin Kirchgatter

Karina Penedo Carvalho

Karine Rezende de Oliveira

Katia da Silva Calabrese

Kelly Aparecida Geraldo Yoneyama Tudini

Kelly Salomão

Kezia Katiani Gorza Scopel

Kildare Rocha de Miranda

Laerte Grisi

Lain Carlos Pontes de Carvalho

Lais de Carvalho

Laura Nogueira da Cruz

Léa Cysne Finkelstein

Lea Ferreira Camillo Coura

Leandro Lemgruber Soares

Leda Quercia Vieira

Leila de Mendonca Lima

Leíla Inês de Aguiar Raposo da Câmara Coelho

Leonardo de Souza Rocha

Leonardo Freire de Lima

Leonardo José de Moura Carvalho

Leonidas Deane

Leonor Laura Pinto Leon
1

1

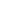

(n)

2

1

1

1

2

2

1

1

1

1

1

1

1

1

1

1

2

2

1

1

1

2

1

2

2

1

1

1

1

2

1

Supplementary data 


\begin{tabular}{|c|c|c|c|}
\hline Name & $1 / 2$ & Name & $1 / 2$ \\
\hline Leucio Camara Alves & 2 & Manoel Paes de Oliveira Neto & 1 \\
\hline Lia Carolina Almeida Soares de Medeiros & 1 & Mara Silvia Carvalhaes & 1 \\
\hline Liana Maria Cardoso Verinaud & 1 & Marcel Ivan Ramirez Araya & 1 \\
\hline Lileia Gonçalves Diotaiuti & 1 & Marcel Teixeira & 1 \\
\hline Liliam de Oliveira Faria Maçaneiro & 1 & Marcela Barbosa de Figueiredo & 1 \\
\hline Lilian Lacerda Bueno & 1 & Marcela de Freitas Lopes & 1 \\
\hline Liliane Coelho da Rocha Nery & 1 & Marcello Andre Barcinski & 2 \\
\hline Lizandra Guidi Magalhaes & 2 & Marcello Xavier Sampaio & 1 \\
\hline Lourdes Maria Garcez dos Santos & 1 & Marcelo da Silva Genestra & 1 \\
\hline Lucia Helena O'dwyer de Oliveira & 2 & Marcelo Einicker Lamas & 1 \\
\hline Lucia Helena Pinto da Silva & 1 & Marcelo Ribeiro da Silva Briones & 1 \\
\hline Lúcia Maria da Cunha Galvão & 1 & Marcelo Salabert Gonzalez & 1 \\
\hline Lucia Mendonça Previato & 2 & Marcelo Urbano Ferreira & 1 \\
\hline Luciamare Perinetti Alves Martins & 1 & Marcelo Vidigal Caliari & 1 \\
\hline Luciana da Conceição Pinto & 1 & Marcia Almeida de Melo & 1 \\
\hline Luciana de Almeida Silva & 1 & Marcia Aparecida Speranca & 1 \\
\hline Luciana de Oliveira Andrade & 1 & Marcia Attias & 1 \\
\hline Luciana Lima & 1 & Marcia Benedita de Oliveira Silva & 1 \\
\hline Luciana Lopes de Almeida Ribeiro Garzoni & 1 & Márcia Cristina Aquino Teixeira & 1 \\
\hline Luciana Regina Meireles Jaguaribe Ekman & 1 & Marcia Cristina Paes & 1 \\
\hline Luciana Ribeiro Garzoni & 1 & Márcia Kiyoe Shimada & 1 \\
\hline Luciana Rodrigues de Almeida & 1 & Marcia Pereira de Oliveira & 1 \\
\hline Luciana Teixeira Zimmermann & 1 & Marcia Rosa de Oliveira & 1 \\
\hline Luciana Urbano dos Santos & 1 & Marcio Silva & 1 \\
\hline Luciane Moreno Storti de Melo & 1 & Marco Aurelio Krieger & 1 \\
\hline Luciano Andrade Moreira & 2 & Marco Túlio Alves da Silva & 1 \\
\hline Lucile Maria Floeter-Winter & 2 & Marco Tulio Antonio Garciazapata & 2 \\
\hline Lucilene Amorim Silva & 1 & Marcos Andre Vannier dos Santos & 1 \\
\hline Lucimeire Antonelli da Silveira & 1 & Marcos Augusto Grigolin Grisotto & 1 \\
\hline Lucio Andre Viana Dias & 1 & Marcos Barbosa de Souza & 1 \\
\hline Lucio Ayres Caldas & 1 & Marcos Gonzaga dos Santos & 1 \\
\hline Lucio Holanda Gondim de Freitas Junior & 1 & Marcos Henrique Ferreira Sorgine & 1 \\
\hline Lúcio Roberto Cançado Castellano & 1 & Marcos Horacio Pereira & 1 \\
\hline Lucy Megumi Yamauchi Lioni & 1 & Marcos José Marques & 1 \\
\hline Ludmila Rodrigues Pinto Ferreira & 1 & Marcos Pinheiro Franque & 2 \\
\hline Luis Carlos Crocco Afonso & 1 & Marcos Rommel Cabanillas Silva & 1 \\
\hline Luis Eduardo Ramirez Giraldo & 1 & Marcos Takashi Obara & 1 \\
\hline Luís Fernando Santana & 1 & Marcos Vinícius da Silva & 2 \\
\hline Luisa Damazio & 1 & Marcus Fernandes de Oliveira & 1 \\
\hline Luiz Alberto Santana & 2 & Maria Aparecida da Gloria Faustino & 1 \\
\hline Luiz Antonio Rodrigues de Freitas & 1 & Maria Aparecida Gomes & 1 \\
\hline Luiz Carlos Alves & 2 & Maria Auxiliadora de Sousa & 1 \\
\hline Luiz Claudio Miletti & 1 & Maria Beatriz Araújo Silva & 1 \\
\hline Luiz Dione Barbosa de Melo & 1 & Maria Carolina Quartim Barbosa Elias & 1 \\
\hline Luiz Fabiano Borges Oliveira & 1 & Maria Cecilia Di Ciero Fernandes & 1 \\
\hline Luiz Fernando Rocha Ferreira da Silva & 2 & Maria Cristina Machado Motta & 1 \\
\hline Luiz Hildebrando Pereira da Silva & 1 & Maria Cristina Nonato & 1 \\
\hline Luis Marcelo Aranha Camargo & 1 & Maria da Gloria Bonecini de Almeida & 2 \\
\hline Luiz Ricardo Orsini Tosi & 2 & Maria da Gloria Fernandes Pegado & 1 \\
\hline Luiz Roberto Sardinha & 1 & Maria das Graças Costa Alecrim & 1 \\
\hline Luiz Rodolpho Raja Gabaglia Travassos & 2 & Maria das Graças Prianti & 1 \\
\hline Luiz Vicente Rizzo & 2 & Maria de Fatima Ferreira da Cruz & 2 \\
\hline Luzia Helena Carvalho & 1 & Maria de Fatima Freire de Melo Ximenes & 1 \\
\hline Luzineide Wanderley Tinoco & 1 & Maria de Fátima Madeira & 1 \\
\hline Lysangela Ronalte Alves & 1 & Maria de Fátima Martins Horta & 1 \\
\hline Magdalena Nascimento Renné & 1 & Maria de Lourdes Petrillo Peixoto & 1 \\
\hline Maira Cegatti Bosetto Mota & 1 & Maria de Nazaré Correia Soeiro & 1 \\
\hline Manoel Barral Netto & 1 & Maria de Nazaré Tavares da Silva & 1 \\
\hline Manoel Otávio da Costa Rocha & 2 & Maria de Nazareth Silveira Leal de Meirelles & 1 \\
\hline
\end{tabular}




\begin{tabular}{|c|c|c|c|}
\hline Name & $1 / 2$ & Name & $1 / 2$ \\
\hline Maria do Socorro Rosa Rodrigues de Carvalho & 1 & Miriam Cristina Leandro Dorta & 1 \\
\hline Maria Esther Carvalho & 1 & Miriam Silva Rafael & 1 \\
\hline Maria Fernanda Laranjeira da Silva & 1 & Mirian Claudia de Souza Pereira & 1 \\
\hline Maria Imaculada Muniz Barboza Junqueira & 1 & Moacir Paranhos Silva & 1 \\
\hline Maria Isabel Lynch Gaete & 1 & Monamaris Marques Borges & 1 \\
\hline Maria Jania Teixeira & 1 & Mônica Cristina de Moraes Silva Bonfim & 1 \\
\hline Maria Julia Manso Alves & 1 & Monica Lucia Gomes & 1 \\
\hline Maria Letícia Costa Reis & 1 & Mucio Flavio Barbosa Ribeiro & 2 \\
\hline Maria Lúcia Vieira Moreno & 1 & Nádia Regina Pereira Almosny & 2 \\
\hline Maria Luiza Brito de Sousa Atta & 1 & Nagila Francinete Costa Secundino & 1 \\
\hline Maria Norma Melo & 1 & Nara Amalia da Rosa Farias & 1 \\
\hline Maria Olívia Amado Ramos Bacellar & 1 & Narcisa Leal da Cunha-E-Silva & 1 \\
\hline Maria Pilar Iribar Calvo & 1 & Nataly Araújo de Saouza & 1 \\
\hline Maria Regina Lima & 1 & Nayara de Oliveira Belo & 1 \\
\hline Maria Regina Reis Amendoeira & 1 & Neci Matos Soares & 1 \\
\hline Maria Stela Branquinho Beaudoin & 1 & Neide Maria da Silva & 1 \\
\hline Maria Terezinha Bahia & 1 & Nilton Barnabe Rodrigues & 2 \\
\hline Maria Valdrinez Campana Lonardoni & 1 & Nitza Maria Alencar Gomes & 1 \\
\hline Maria Deane & 2 & Nobuko Yoshida & 1 \\
\hline Mariana Caldas Waghabi & 1 & Noemi Nosomi Taniwaki & 1 \\
\hline Mariana Machado Hecht & 1 & Octavio Fernandes da Silva Filho & 1 \\
\hline Mariangela Carneiro & 2 & Odair Genaro & 1 \\
\hline Mariano Gustavo Zalis & 1 & Olindo Assis Martins Filho & 2 \\
\hline Mariko Yokoo & 1 & Osmarina Pereira da Paixão & 1 \\
\hline Marilene Maria de Lima & 1 & Osvaldo Marinotti & 2 \\
\hline Marilene Suzan Marques Michalick & 1 & Osvaldo Pompilio de Melo Neto & 2 \\
\hline Marinete Marins Povoa & 1 & Otavio Henrique Thiemann & 1 \\
\hline Mario E Camargo & 2 & Otilio Machado Pereira Bastos & 1 \\
\hline Mário Steindel & 1 & Pamela Orjuela Sánchez & 1 \\
\hline Marise Pinheiro Nunes & 1 & Patrícia Alves Morking & 1 \\
\hline Maristela Gomes da Cunha & 1 & Patricia Cuervo Escobar & 1 \\
\hline Mariza Martins Avelino & 1 & Patricia de Azambuja Penna & 1 \\
\hline Marlene Benchimol & 1 & Patricia de Oliveira Camera & 1 \\
\hline Marlene Cabrine dos Santos Silva & 1 & Patrícia Fampa Negreiros Lima & 1 \\
\hline Marlene Isabel Vargas Viloria & 1 & Patricia Flávia Quaresma & 1 \\
\hline Marlisson Augusto Costa Feitosa & 1 & Patrícia Giupponi Cardoso & 1 \\
\hline Marta Chagas Monteiro & 1 & Patrícia Karla Santos Ramos & 1 \\
\hline Marta de Lana & 1 & Patrícia Massara Martinelli & 1 \\
\hline Marta Maria Geraldes Teixeira & 1 & Patricia Sampaio Tavares Veras & 1 \\
\hline Marta Tavares D'agosto & 1 & Patricia Soares de Maria de Medeiros & 1 \\
\hline Marta Teixeira Gomes & 1 & Paula Melo de Abreu Vieira & 1 \\
\hline Martha Cecilia Suárez Mutis & 1 & Paulo Afonso Nogueira & 1 \\
\hline Mauricio Martins Rodrigues & 1 & Paulo Batista de Carvalho & 1 \\
\hline Maurilio José Soares & 1 & Paulo César Augusto de Souza & 1 \\
\hline Mauro Celio de Almeida Marzochi & 2 & Paulo de Tarso Gonçalves Leopoldo & 1 \\
\hline Mauro Ferreira de Azevedo & 1 & Paulo Eduardo Martins Ribolla & 2 \\
\hline Mauro Javier Cortez Véliz & 1 & Paulo Filemon Paolucci Pimenta & 1 \\
\hline Mauro Shugiro Tada & 1 & Paulo Henrique da Costa Pinheiro & 1 \\
\hline Max Jean de Ornelas Toledo & 1 & Paulo Paes de Andrade & 1 \\
\hline Mayra Xiomara Hernández Sanabria & 1 & Paulo Roberto Lima Machado & 1 \\
\hline Meire Ioshie Hiyane & 1 & Pedro Lagerblad de Oliveira & 1 \\
\hline Michel Pinkus Rabinovitch & 2 & Pedro Roosevelt Torres Rom $\sqrt{£ O}$ & 1 \\
\hline Michelle Aparecida Ribeiro de Freitas & 1 & Pedro Luiz Silva Pinto & 1 \\
\hline Michelle Frazão Muzitano & 1 & Phileno Pinge Filho & 1 \\
\hline Michelle Tanny Cunha do Nascimento & 1 & Philip Davis Marsden & 2 \\
\hline Milena Botelho Pereira Soares & 1 & Phillippe Grelier & 1 \\
\hline Milena de Paiva Cavalcanti & 1 & Piero Bagnaresi & 1 \\
\hline Milton Adriano Pelli de Oliveira & 1 & Priscila Carneiro Campos & 1 \\
\hline Miria Gomes Pereira & 1 & Priscila Grynberg & 1 \\
\hline
\end{tabular}




\begin{tabular}{|c|c|c|c|}
\hline Name & $1 / 2$ & Name & $1 / 2$ \\
\hline Rafael Dhalia & 1 & Rosa Amelia Maldonado Medina & 1 \\
\hline Rafael Maciel de Freitas & 2 & Rosa Maria Esteves Arantes & 2 \\
\hline Rafael Miyazawa Martins & 1 & Rosa Maria Tubaki & 1 \\
\hline Rafael Otávio Cançado Motta & 1 & Rosa Teixeira de Pinho & 1 \\
\hline Rafael Veríssimo Monteiro & 1 & Rosália Morais Torres & 1 \\
\hline Rafael Victorio Carvalho Guido & 1 & Rosana Alves Martorelli & 1 \\
\hline Raimunda Nonata Ribeiro Sampaio & 1 & Rosana de Sousa Veras & 1 \\
\hline Raimundo Wilson de Carvalho & 2 & Rosane Maria Temporal & 1 \\
\hline Raissa Bocchi Pedroso & 1 & Rosangela Locatelli Dittrich & 2 \\
\hline Raquel da Silva Pacheco & 1 & Rosângela Maria Rodrigues & 2 \\
\hline Raquel Elisa da Silva López & 1 & Rosangela Zacarias Machado & 1 \\
\hline Raquel Ferreira Rodrigues & 1 & Rosiane Aparecida da Silva & 1 \\
\hline Raymundo Martins de Castro & 2 & Rossiane Claudia Vommaro & 1 \\
\hline Regiane Mathias & 1 & Rozalia Mendes Figueira Campos & 1 \\
\hline Regina Celia Bressan Queiroz de Figueiredo & 1 & Rubem Figueiredo Sadok Menna Barreto & 1 \\
\hline Regina de Fatima dos Santos Braz & 1 & Sabrina Castilho Duarte & 1 \\
\hline Regina Helena Saramago Peralta & 2 & Salvatore Giovanni de Simone & 1 \\
\hline Regina Maria Barretto Cicarelli & 1 & Samanta Etel Treiger Borborema & 1 \\
\hline Regina Maura Bueno Franco & 1 & Samuel Goldenberg & 2 \\
\hline Regina Mitsuka Breganí & 1 & Samuel Pessoa & 2 \\
\hline Reginaldo Peçanha Brazil & 1 & Sandra Mayumi Nishi & 1 \\
\hline Regis Bernardo Brandim Gomes & 1 & Sandro Nelson Lunedo & 1 \\
\hline Renata de Meirelles Santos Pereira & 1 & Sanóvia Aparecida de Lima Pereira & 1 \\
\hline Renata Oliveira de Araújo Soares & 1 & Santuza Maria Ribeiro Teixeira & 2 \\
\hline Renata Rosito Tonelli & 1 & Sara Timoteo Passos & 1 \\
\hline Renata Tomé Alves & 1 & Saulo Cabral Bourguignon & 1 \\
\hline Renata Tonhosolo & 1 & Saulo Teixeira de Moura & 1 \\
\hline Renata Torres de Souza & 1 & Sebastião Aldo da Silva Valente & 1 \\
\hline Renato Arruda Mortara & 2 & Selene Maia de Morais & 2 \\
\hline Renato Augusto Damatta & 2 & Selma Margareth Bacellar de Souza Leã & 1 \\
\hline Renato Sathler Avelar & 1 & Selma Giorgio & 1 \\
\hline Ricardo Andrade Barata & 1 & Selma Maria Bezerra Jeronimo & 2 \\
\hline Ricardo Gonçalves & 1 & Sergian Vianna Cardozo & 1 \\
\hline Ricardo Lourenço de Oliveira & 1 & Sergio Danilo Junho Pena & 2 \\
\hline Ricardo Ribeiro dos Santos & 1 & Sérgio de Albuquerque & 1 \\
\hline Ricardo Scher & 1 & Sergio Galvão Coutinho & 1 \\
\hline Ricardo Toshio Fujiwara & 1 & Sergio Henrique Seabra & 1 \\
\hline Ricardo Tostes Gazzinelli & 1 & Sergio Marcelo Rodriguez Malaga & 1 \\
\hline Ricardo Wagner de Almeida Vitor & 1 & Sergio Schenkman & 2 \\
\hline Ricardo Wagner Dias Portela & 1 & Sheila Cristina Nardelli & 1 \\
\hline Rinaldo Aparecido Mota & 1 & Silvana Maria Eloi Santos & 2 \\
\hline Rita de Cássia Moreira de Souza & 1 & Silvana Marques de Araujo & 1 \\
\hline Rita de Cássia Pontello Rampazzo & 1 & Silvane Maria Fonseca Murta & 1 \\
\hline Riva de Paula Oliveira & 1 & Silvia Amaral Gonçalves da Silva & 1 \\
\hline Roberta Dias Rodrigues Rocha & 1 & Silvia Beatriz Boscardin & 1 \\
\hline Roberta Lemos Freire & 1 & Silvia Ermelinda Barbosa & 1 \\
\hline Roberto Esteves Pires Castanho & 1 & Sílvia Maria Santos Carvalho & 1 \\
\hline Roberto José da Silva Badaró & 1 & Silvia Maria Spalding & 1 \\
\hline Roberto Mitsuyoshi Hiramoto & 1 & Silvia Reni Bortolin Uliana & 1 \\
\hline Roberto Rudge de Moraes Barros & 1 & Silvio Luís Pereira de Souza & 1 \\
\hline Robson Amaro Augusto da Silva & 1 & Silvio Santana Dolabella & 1 \\
\hline Rodolfo Cordeiro Giunchetti & 1 & Simone Cardoso Leon & 1 \\
\hline Rodrigo Correa de Oliveira & 2 & Simone da Fonseca Pires & 1 \\
\hline Rodrigo Costa da Silva & 1 & Simone Ladeia Andrade & 1 \\
\hline Rodrigo Pedro Pinto Soares & 1 & Simone Patrícia Carneiro de Freitas & 1 \\
\hline Rogério Luiz Kopp & 1 & Sinval Pinto Brandão Filho & 1 \\
\hline Rogerio Saad Vaz & 1 & Solange Lisboa de Castro & 1 \\
\hline Rômulo Menna Barreto Valença & 1 & Sônia de Lucena Mioranza & 1 \\
\hline Ronize Andreia Ferreira & 1 & Sonia Gumes Andrade & 1 \\
\hline
\end{tabular}




\begin{tabular}{|c|c|c|c|}
\hline Name & $1 / 2$ & Name & $1 / 2$ \\
\hline Sonia Maria Neumann Cupolilo & 1 & Vanete Thomaz Soccol & 1 \\
\hline Sônia Rolim Reis & 1 & Vania Lucia Brandao Nunes & 1 \\
\hline Sonia Soares Costa & 2 & Vanize de Oliveira Macêdo & 1 \\
\hline Stefano Trapani & 1 & Vera da Costa Valente & 1 \\
\hline Stella Maria Barrouin Melo & 1 & Vera Lucia Pereira-Chioccola & 1 \\
\hline Stenio Perdigão Fragoso & 1 & Veronica Figueiredo do Amaral & 1 \\
\hline Sueli Fumie Yamada Ogatta & 1 & Veronica Schmitz Pereira & 1 \\
\hline Suzana Passos Chaves & 1 & Vicente de Paulo Coelho Peixoto de Toledo & 1 \\
\hline Suzete Araujo Oliveira Gomes & 1 & Vinícius Santana Nunes & 1 \\
\hline Sylvio Celso Gonçalves da Costa & 1 & Virginia Maria Barros de Lorena & 1 \\
\hline Taís Nóbrega de Sousa & 1 & Virgínia Maria Góes da Silva & 1 \\
\hline Tamara Nunes de Lima Camara & 1 & Virmondes Rodrigues Junior & 1 \\
\hline Tania Cremonini de Araujo-Jorge & 1 & Viviane Rodrigues Esperandim & 1 \\
\hline Tania Ueda Nakamura & 1 & Viviane Sampaio Boaventura de Oliveira & 1 \\
\hline Tânia Zaverucha do Valle & 1 & Vladimir Michailowsky Leite Ribeiro & 1 \\
\hline Tatiana Galvão de Melo de Oliveira & 1 & Vlaudia Maria Assis Costa & 1 \\
\hline Tatiana Rodrigues de Moura & $\begin{array}{l}1 \\
1\end{array}$ & Wagner Barbosa Dias & 1 \\
\hline Tatiana Shioji Tiuman & $\begin{array}{l}1 \\
1\end{array}$ & Wagner Luiz Tafuri & 1 \\
\hline Tatjana Keesen de Souza Lima & 1 & Wagner Welber Arrais da Silva & 1 \\
\hline $\begin{array}{l}\text { latjana Keesen de Souza Lima } \\
\text { Tecia Maria Ulisses de Carvalho }\end{array}$ & 1 & Walderez Ornelas Dutra & 1 \\
\hline $\begin{array}{l}\text { Tecia Maria Ulisses de Carvalho } \\
\text { Teresa Cristina Bergamo do Bomfim }\end{array}$ & 1 & Walter Ceretti Junior & 1 \\
\hline $\begin{array}{l}\text { Teresa Cristina Bergamo do Bomfim } \\
\text { Teresa Cristina Franca de Assumpcão }\end{array}$ & 1 & Walter Colli & 2 \\
\hline & 1 & Walter Flausino & 1 \\
\hline Teresa Cristina Leandro de Jesus & 1 & Wander Rogerio Pavanelli & 1 \\
\hline Teresa Cristina Monte Gonçalves & 1 & Wanderley de Souza & 1 \\
\hline Teresa Fernandae Silva do Nascimento & 1 & Wanderli Pedro Tadei & 2 \\
\hline Tereza Cristina Orlando & 1 & Wanderson Duarte da Rocha & 1 \\
\hline Thais Cristina Baeta Soares Souto Padron & 1 & Wanderson Geraldo de Lima & 1 \\
\hline Thais Ferreira Isabel & 1 & Washington Luis Conrado dos Santos & 2 \\
\hline Thais Gomes Verzignassi Silveira & 1 & Washington Luiz Tafuri & 2 \\
\hline Thales de Brito & 2 & Wendel Coura Vital & 1 \\
\hline Thiago de Castro Gomes & 1 & Wendell Sérgio Ferreira Meira & 1 \\
\hline Tiago Moreno Castilho & 1 & William Mello Kattenbach & 1 \\
\hline Tiago Wilson Patriarca Mineo & 1 & Williams Jorge da Cruz Macêdo & 1 \\
\hline Trícia Maria Ferreira de Sousa Oliveira & 1 & Wilson Jacinto Silva de Souza & 1 \\
\hline Ulisses Gazos Lopes & 1 & Wilson Savino & 2 \\
\hline Vagner José Mendonça & 1 & Wim Maurits Sylvain Degrave & 2 \\
\hline Valdir de Queiroz Balbino & 1 & Wladimir Lobato Paraense & 2 \\
\hline Valéria Marçal Felix de Lima & 2 & Yara de Miranda Gomes & 1 \\
\hline Valter Ferreira de Andrade Neto & 1 & Yara Maria Traub-Csekö & 2 \\
\hline Vanessa Cabreira de Freitas & 1 & Zelia Maria Profeta da Luz & 1 \\
\hline Vanessa Migliaccio & 1 & Zelson Giacomo Líss & 1 \\
\hline Vanessa Peruhype Magalhães Pascoal & 1 & Zigman Brener & 2 \\
\hline
\end{tabular}

bolded names: persons who developed $\mathrm{PhD}$ in protozoology having as supervisor a nonprotozoologist. 
TABLE V

Year that persons who did not develop $\mathrm{PhD}$ in protozoology published in this field

\begin{tabular}{|c|c|c|c|c|c|}
\hline Name & Year $^{a}$ & ID & Name & Year $^{a}$ & ID \\
\hline Adrian Martin Pohlit & 2007 & 1 & Hector Barrabin & 2000 & 57 \\
\hline Adriano Defini Andricopulo & 2002 & 2 & Helio Langoni & 1999 & 58 \\
\hline Alejandro Miguel Katzin & 1986 & 3 & Hernando Antonio del Portillo Obando & 1987 & 59 \\
\hline Alessandra D'almeida Filardy & 2011 & 4 & Hertha Meyer & 1948 & 60 \\
\hline Alessandra Scofield & 2000 & 5 & Hooman Momen & 1990 & 61 \\
\hline Alexandre Afranio Peixoto & 2001 & 6 & Hugo Caire de Castro Faria Neto & 2003 & 62 \\
\hline Aluízio Prata & 1963 & 7 & Humberto de Araújo Rangel & 1979 & 63 \\
\hline Ana Carolina Campi Azevedo & 2012 & 8 & Isaac Roitman & 1969 & 64 \\
\hline Ana Cristina da Silva Pinto & 2007 & 9 & Jane Lima dos Santos & 2006 & 65 \\
\hline Ana Lúcia Teles Rabello & 1999 & 10 & Jayme Angluster & 1985 & 66 \\
\hline Andréa Teixeira de Carvalho & 2002 & 11 & Jayme Neves & 1961 & 67 \\
\hline Angela Kaysel Cruz & 1990 & 12 & Jeffrey Jon Shaw & 1965 & 68 \\
\hline Amilcar Viana Martins & 1956 & 13 & Jorge Elias Kalil Filho & 1982 & 69 \\
\hline Anibal Antonio da Silva Pereira & 1979 & 14 & José Manuel Macário Rebêlo & 1996 & 70 \\
\hline Anita Hilda Straus Takahashi & 1993 & 15 & José Ailton da Silva & 2008 & 71 \\
\hline Antonio de Oliveira Lima & 1989 & 16 & Jose Daniel Figueroa-Villar & 1995 & 72 \\
\hline Antonio Florencio de Figueiredo & 2007 & 17 & Jose Maria Alvarez Mosig & 1988 & 73 \\
\hline Astolpho Ferraz de Siqueira & 1963 & 18 & José Maria de Souza & 1982 & 74 \\
\hline Bartira Rossi Bergmann & 1987 & 19 & Jose Mauro Peralta & 1980 & 75 \\
\hline Beatriz Dolabela de Lima & 1996 & 20 & Jose Osvaldo Previato & 1979 & 76 \\
\hline Beatriz Rossetti Ferreira & 2005 & 21 & Jose Roberto Meyer Fernandes & 1996 & 77 \\
\hline Bernardo Galvão Castro Filho & 1979 & 22 & Jose Rodrigues da Silva & 1957 & 78 \\
\hline Bianca Silvana Zingales & 1979 & 23 & Joseli Lannes-Vieira & 1986 & 79 \\
\hline Carlos Alberto Pereira Tavares & 1992 & 24 & Juliana Lopes Rangel Fietto & 2004 & 80 \\
\hline Carlos Chagas & 1909 & 25 & Julio Pudles & 1995 & 81 \\
\hline Carlos Eduardo Pereira Corbett & 1984 & 26 & Julio Scharfstein & 1982 & 82 \\
\hline Carlos Eduardo Tosta da Silva & 1982 & 27 & Laerte Grisi & 1976 & 83 \\
\hline Carlos Mauricio de Figueiredo Antunes & 1985 & 28 & Lain Carlos Pontes de Carvalho & 1985 & 84 \\
\hline Carlos Medicis Morel & 1977 & 29 & Lea Ferreira Camillo Coura & 1993 & 85 \\
\hline Carlos Renato Machado & 2001 & 30 & Leda Quercia Vieira & 1993 & 86 \\
\hline Carlos Wilson Gomes Lopes & 1980 & 31 & Leila de Mendonca Lima & 2007 & 87 \\
\hline Célia Regina da Silva Garcia & 1995 & 32 & Leonidas Deane & 1955 & 88 \\
\hline Celuta Sales Alviano & 1980 & 33 & Leucio Camara Alves & 2001 & 89 \\
\hline Cezar Martins de Sá & 1980 & 34 & Lizandra Guidi Magalhaes & 2011 & 90 \\
\hline Claudia Maria Leal Bevilaqua & 1998 & 35 & Lucia Helena O’Dwyer de Oliveira & 1997 & 91 \\
\hline Claudio Tadeu Daniel-Ribeiro & 1981 & 36 & Lucia Mendonça Previato & 1986 & 92 \\
\hline Clea Nazare Carneiro Bichara & 2004 & 37 & Luciano Andrade Moreira & 2000 & 93 \\
\hline Conceiçao Ribeiro da Silva Machado & 1975 & 38 & Lucile Maria Floeter-Winter & 1990 & 94 \\
\hline Cristiana Ferreira Alves de Brito & 2005 & 39 & Luiz Alberto Santana & 2001 & 95 \\
\hline Débora Pereira Garcia Melo & 2006 & 40 & Luiz Carlos Alves & 2013 & 96 \\
\hline Denise da Silveira Lemos & 2008 & 41 & Luiz Fernando Rocha Ferreira da Silva & 2004 & 97 \\
\hline Edna Freymuller Haapalainen & 1977 & 42 & Luiz Ricardo Orsini Tosi & 1996 & 98 \\
\hline Edson Rondinelli & 1984 & 43 & Luiz Rodolpho Raja Gabaglia Travassos & 1986 & 99 \\
\hline Eloy Guillermo Castellón Bermúdez & 1989 & 44 & Luiz Vicente Rizzo & 2004 & 100 \\
\hline Erney Felicio Plessmann de Camargo & 1961 & 45 & Manoel Otávio da Costa Rocha & 2002 & 101 \\
\hline Eveline Gomes Vasconcelos & 2001 & 46 & Marcello Andre Barcinski & 1984 & 102 \\
\hline Fernando Dias de Avila Pires & 1998 & 47 & Marco Tulio Antonio Garciazapata & 1985 & 103 \\
\hline Firmino Torres de Castro & 1977 & 48 & Marcos Pinheiro Franque & 2004 & 104 \\
\hline Francisco Juarez Ramalho Pinto & 1994 & 49 & Marcos Vinícius da Silva & 2010 & 105 \\
\hline Frederico Guilherme Coutinho Abath & 1986 & 50 & Maria da Gloria Bonecini de Almeida & 2009 & 106 \\
\hline George Alexandre dos Reis & 1980 & 51 & Maria de Fatima Ferreira da Cruz & 2003 & 107 \\
\hline Gerhard Wunderlich & 1999 & 52 & Maria Vos Paumgartten Deane & 1955 & 108 \\
\hline Glaucius Oliva & 1988 & 53 & Mariangela Carneiro & 2009 & 109 \\
\hline Gloria Regina Franco & 2006 & 54 & Marinete Marins Povoa & 1994 & 110 \\
\hline Guilherme Loureiro Werneck & 2002 & 55 & Mario E Camargo & 1964 & 111 \\
\hline Hatisaburo Masuda & 1979 & 56 & Mauro Celio de Almeida Marzochi & 1980 & 112 \\
\hline
\end{tabular}




\begin{tabular}{|c|c|c|c|c|c|}
\hline Name & Year $^{a}$ & ID & Name & Year $^{a}$ & ID \\
\hline Michel Pinkus Rabinovitch & 1982 & 113 & Samuel Goldenberg & 1984 & 135 \\
\hline Mucio Flavio Barbosa Ribeiro & 2012 & 114 & Samuel Pessoa & 1943 & 136 \\
\hline Nádia Regina Pereira Almosny & 2012 & 115 & Santuza Maria Teixeira & 1994 & 137 \\
\hline Nilton Barnabé Rodrigues & 2015 & 116 & Selene Maia de Morais & 2006 & 138 \\
\hline Olindo Assis Martins Filho & 1999 & 117 & Selma Maria Bezerra Jeronimo & 1992 & 139 \\
\hline Osvaldo Marinotti & 2005 & 118 & Sergio Danilo Junho Pena & 1992 & 140 \\
\hline Osvaldo Pompilio de Melo Neto & 2006 & 119 & Sergio Schenkman & 1986 & 141 \\
\hline Otavio Henrique Thiemann & 2001 & 120 & Silvana Maria Elói Santos & 2002 & 142 \\
\hline Patricia Sampaio Tavares Veras & 2003 & 121 & Sonia Soares Costa & 1999 & 143 \\
\hline Paulo Eduardo Martins Ribolla & 2004 & 122 & Thales de Brito & 1962 & 144 \\
\hline Philip Davis Marsden & 1980 & 123 & Valéria Marçal Felix de Lima & 2002 & 145 \\
\hline Rafael Maciel de Freitas & 2004 & 124 & Vanete Thomaz Soccol & 2001 & 146 \\
\hline Raimundo Wilson de Carvalho & 2003 & 125 & Walter Colli & 1974 & 147 \\
\hline Raymundo Martins de Castro & 1971 & 126 & Wanderli Pedro Tadei & 1982 & 148 \\
\hline Regina Helena Saramago Peralta & 2007 & 127 & Washington Luis Conrado dos Santos & 1993 & 149 \\
\hline Reginaldo Peçanha Brazil & 1983 & 128 & Washington Luiz Tafuri & 1957 & 150 \\
\hline Renato Arruda Mortara & 1988 & 129 & Wilson Savino & 1989 & 151 \\
\hline Renato Augusto Damatta & 2000 & 130 & Wim Maurits Sylvain Degrave & 2003 & 152 \\
\hline Rodrigo Correa de Oliveira & 2002 & 131 & Wladimir Lobato Paraense & 1949 & 153 \\
\hline Rosa Maria Esteves Arantes & 2004 & 132 & Yara de Miranda Gomes & 1995 & 154 \\
\hline Rosangela Locatelli Dittrich & 2001 & 133 & Yara Maria Traub-Cseko & 1988 & 155 \\
\hline Rosângela Maria Rodrigues & 2005 & 134 & Zigman Brener & 1951 & 156 \\
\hline
\end{tabular}

$a$ : the year that scientist published his first paper in Brazil after his PhD; bolded names: persons who did develop PhD in protozoology outside Brazil. 